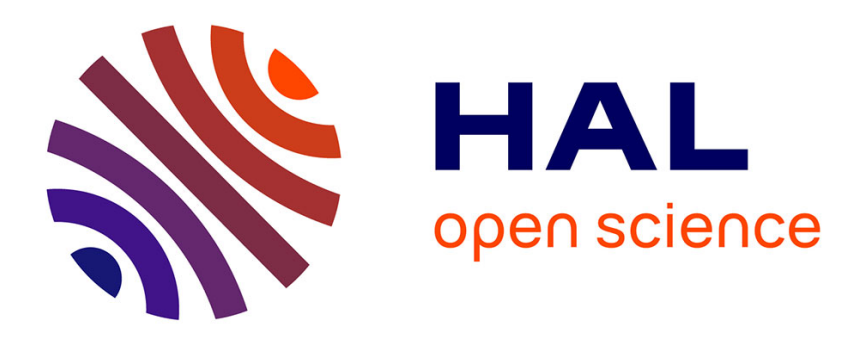

\title{
Enfants conçus avec une aide médicale dans la cohorte Elfe
}

\author{
Claire Poulalhon
}

\section{To cite this version:}

Claire Poulalhon. Enfants conçus avec une aide médicale dans la cohorte Elfe. Médecine humaine et pathologie. 2015. dumas-01252602

\section{HAL Id: dumas-01252602 https://dumas.ccsd.cnrs.fr/dumas-01252602}

Submitted on 7 Jan 2016

HAL is a multi-disciplinary open access archive for the deposit and dissemination of scientific research documents, whether they are published or not. The documents may come from teaching and research institutions in France or abroad, or from public or private research centers.
L'archive ouverte pluridisciplinaire HAL, est destinée au dépôt et à la diffusion de documents scientifiques de niveau recherche, publiés ou non, émanant des établissements d'enseignement et de recherche français ou étrangers, des laboratoires publics ou privés. 


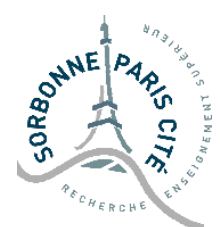

\section{AVERTISSEMENT}

Cette thèse d'exercice est le fruit d'un travail approuvé par le jury de soutenance et réalisé dans le but d'obtenir le diplôme d'Etat de docteur en médecine. Ce document est mis à disposition de l'ensemble de la communauté universitaire élargie.

Il est soumis à la propriété intellectuelle de l'auteur. Ceci implique une obligation de citation et de référencement lors de l'utilisation de ce document.

D’autre part, toute contrefaçon, plagiat, reproduction illicite encourt toute poursuite pénale.

Code de la Propriété Intellectuelle. Articles L 122.4

Code de la Propriété Intellectuelle. Articles L 335.2-L 335.10 


\section{UNIVERSITÉ PARIS DESCARTES \\ Faculté de Médecine PARIS DESCARTES}

Année 2015

\section{THÈSE \\ POUR LE DIPLÔME D’ÉTAT \\ DE DOCTEUR EN MÉDECINE}

Spécialité : Santé publique et Médecine sociale

Enfants conçus avec une aide médicale dans la cohorte Elfe

Présentée et soutenue publiquement

le 29 septembre 2015

Par

Claire POULALHON

Née le 12mars 1986 à Clamart (92)

Dirigée par Mme Élise De La Rochebrochard, Directrice de Recherche

Jury :

M. Le Professeur Bruno Falissard, PU-PH Président

Mme Le Docteur Perrine Capmas, CCA

M. Le Professeur Vincent Gajdos, PU-PH

Mme Le Professeur Laurence Meyer, PU-PH

(c) $(9) \begin{aligned} & \text { Except where otherwise noted, this work is licensed under } \\ & \text { http://creativecommons.org/licenses/by-nc-nd/3.0/ }\end{aligned}$ 
ENFANTS CONÇUS AVEC UNE AIDE MÉDICALE DANS LA COHORTE ELFE 


\section{REMERCIEMENTS}

Cette étude n'aurait pas été possible sans le travail de l'équipe Elfe et des nombreuses équipes de chercheurs qui ont élaboré le protocole de la cohorte Elfe et permis sa mise en place et sa poursuite depuis 2011.

Un grand merci à Élise de La Rochebrochard pour son encadrement énergique et enrichissant qui m'a permis de rebondir face aux multiples péripéties de ce projet!

Je tiens à remercier Jean Bouyer de m'avoir accueillie dans son équipe et d'avoir pu consacrer du temps à notre réflexion, par ses relectures et ses conseils toujours avisés. Merci surtout de m'avoir ouvert les portes de l'unité CESP 1018, à un moment délicat de mon parcours. J'y suis ensuite toujours revenue avec plaisir.

Merci à Fabienne Pessionne et Sabrina Di Constanzo (Agence de la Biomédecine), qui ont accepté de partager leur expérience de l'assistance médicale à la procréation en France et de discuter nos résultats.

Je remercie Bruno Falissard d'avoir accepté de présider le jury de ma thèse, et Laurence Meyer et Perrine Capmas d'avoir accepté d'en faire partie. Merci d'accorder du temps à l'évaluation de mon travail.

Je remercie tout particulièrement Vincent Gajdos, qui grâce à Jean Bouyer a guidé mes premiers pas en santé publique, m'a permis de participer à l'aventure Pronour avec Karen Milcent, et qui aujourd'hui a bien voulu être présent pour la conclusion de mon internat. La boucle est bouclée, et j'en suis très honorée.

Une pensée enfin pour mes collègues Soraya, Moussa et Tatiana. 
À mes parents, frères et sœur, neveux et nièces, à ma grand-mère.

À Jean-Pierre.

À Thomas, qui m'a quelque peu compliqué la tâche, mais pour mon plus grand bonheur! 


\section{Contributeurs à Elfe}

L'enquête Elfe est une réalisation conjointe de l'Institut national d'études démographiques (Ined), de l’Institut national de la santé et de la recherche médicale (Inserm), de l'Établissement français du sang (EFS), de l'Institut de veille sanitaire (InVS), de l'Institut national de la statistique et des études économiques (Insee), de la Direction générale de la santé (DGS, Ministère en charge de la santé), de la Direction générale de la prévention des risques (DGPR, Ministère en charge de l'Environnement), de la Direction de la recherche, des études, de l'évaluation et des statistiques (Drees, Ministères en charge de la santé et de l'emploi) et de la Caisse nationale des allocations familiales (Cnaf), avec le soutien du Ministère de la Recherche, du Comité de concertation pour les données en sciences humaines et sociales (CCDSHS) et du Ministère de la Culture (Deps). Dans le cadre de la plateforme RECONAI, elle bénéficie d'une aide de l'État gérée par l'Agence nationale de la recherche au titre du programme Investissements d'avenir portant la référence ANR-11-EQPX-0038. 



\section{TABLE DES MATIÈRES}

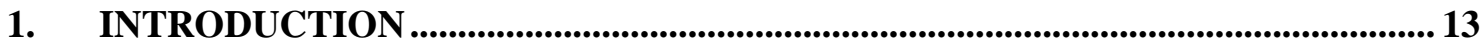

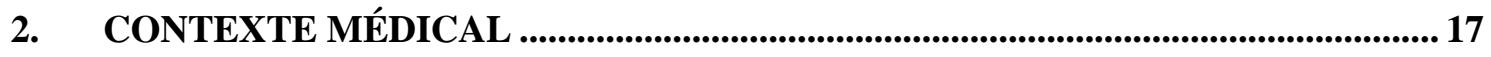

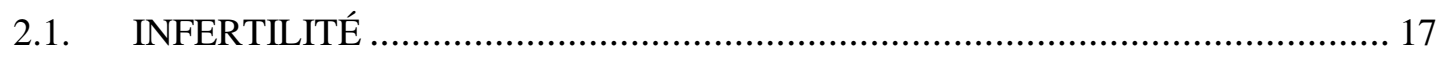

2.2. TRAITEMENTS DE L'INFERTILITÉ _...................................................... 23

2.3. COMPLICATIONS ASSOCIÉES AUX TRAITEMENTS DE L'INFERTILITÉ ... 28

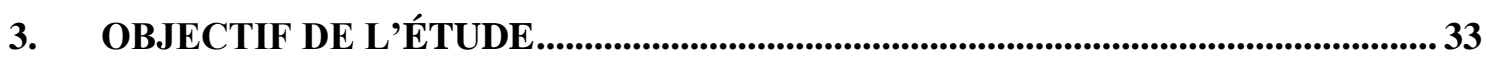

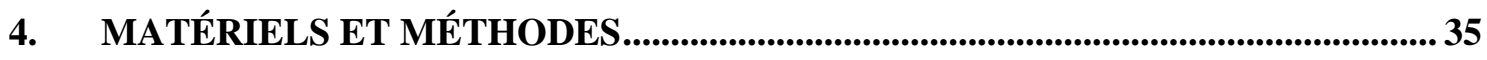

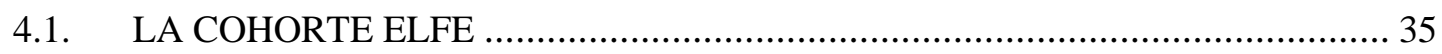

4.2. POPULATION D'ÉTUDE : approche avec trois groupes d'étude........................ 43

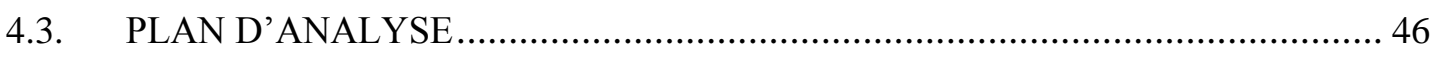

4.3.1. Caractéristiques de la population d'étude........................................................... 46

4.3.2. Exploration de deux indicateurs de santé à la naissance ...................................... 47

4.3.3. Note sur la pondération dans Elfe et l'analyse des données pondérées..................51

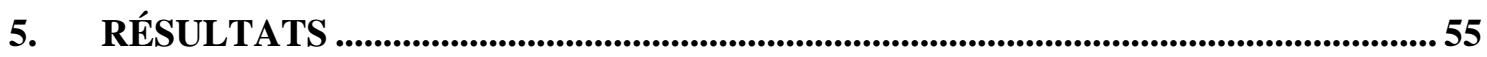

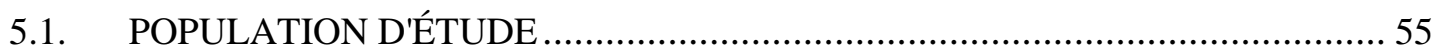

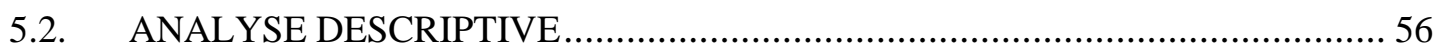

5.2.1. Caractéristiques sociodémographiques parentales ............................................. 56

5.2.2. Antécédents maternels et contexte de la grossesse ................................................5 58

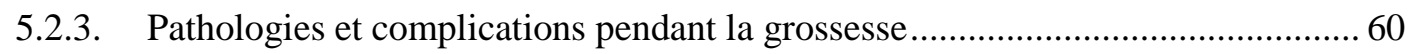

5.2.4. Travail et accouchement, état de santé des femmes et des enfants à la naissance.61

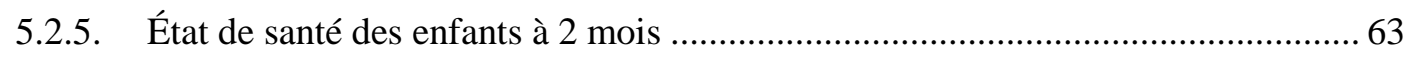

5.2.6. Description par sous-groupes de traitement de l'infertilité ...................................6 65 


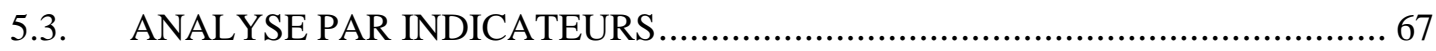

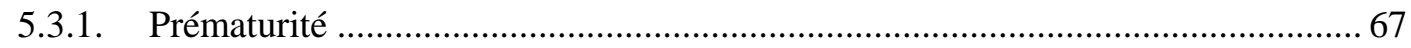

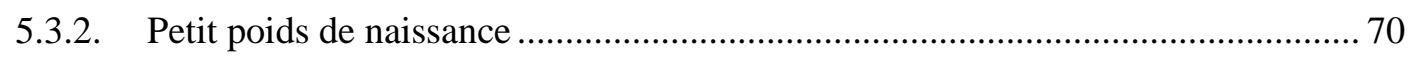

5.3.3. Analyse en sous-groupes de traitement de l'infertilité .......................................... 73

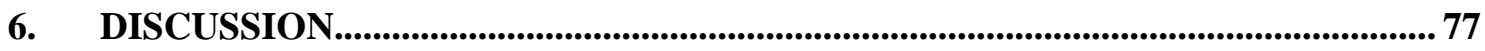

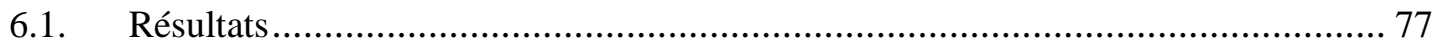

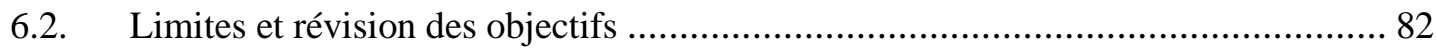

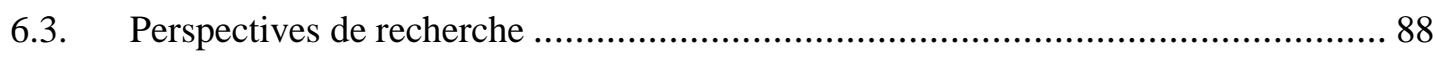

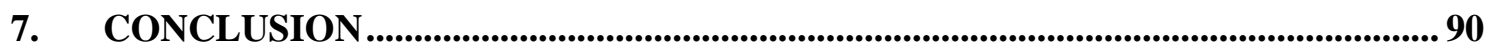

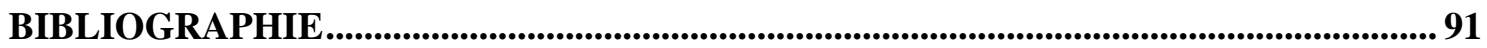

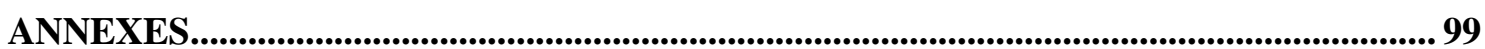




\section{LISTE DES SIGLES ET ABRÉVIATIONS UTILISÉS}

ADN

AME

AMP

ARN

BAS

BEP

CAP

CDI

CMU

CMV

CSP

Elfe

ENP

EPIPAGE

FIV

FSH

HTA

IAC

IAD

IC

ICSI

IMC

IMG

Insee

InVS

IVG
Acide DésoxyriboNucléique

Aide Médicale d'État

Assistance Médicale à la Procréation

Acide RiboNucléique

British Ability Scales

Brevet d'Études Professionnelles

Certificat d'Aptitude Professionnelle

Child Development Inventory

Couverture Maladie Universelle

CytoMégaloVirus

Catégorie SocioProfessionnelle

Étude longitudinale française depuis l'enfance

Enquête Nationale Périnatale

étude ÉPIdémiologique sur les Petits Âges GEstationnels

Fécondation In Vitro

Follicle Stimulating Hormone (hormone folliculo-stimulante)

HyperTension Artérielle

Insémination Artificielle avec spermatozoïdes du Conjoint

Insémination Artificielle avec spermatozoïdes de Donneur

Intervalle de Confiance

IntraCytoplasmic Sperm Injection

Indice de Masse Corporelle

Interruption Médicale de Grossesse

Institut national de la statistique et des études économiques

Institut national de Veille Sanitaire

Interruption Volontaire de Grossesse 
LH Luteinizing Hormone (hormone lutéinisante)

OATS Oligo-Asthéno-Térato-Spermie

Obseff Observatoire épidémiologique de la fertilité en France

OMS Organisation Mondiale de la Santé

OR Odds Ratio

RCIU Retard de Croissance Intra-Utérin

SA Semaine d'Aménorrhée

SOPK Syndrome des Ovaires PolyKystiques

TEC Transfert d'Embryon Congelé

VIH Virus de l'Immunodéficience Humaine 


\section{LISTE DES TABLEAUX}

Tableau 1 : Principales étapes du suivi pour les huit premières années de la cohorte Elfe

Tableau 2: Quelques statistiques des naissances dans Elfe et l'ENP 2010

Tableau 3 : Caractéristiques socio-économiques parentales selon le contexte de la conception

Tableau 4 : Antécédents maternels et contexte de la grossesse selon le contexte de la conception

Tableau 5: Pathologies et complications de la grossesse selon le contexte de la conception

Tableau 6 : Travail et accouchement, état de santé des mères et des enfants à la naissance et en suites de couches immédiates selon le contexte de la conception

Tableau 7 : État de santé des enfants à deux mois selon le contexte de la conception .....

Tableau 8: Travail et accouchement, état de santé des mères et des enfants à la naissance et en suites de couches selon le groupe de traitement de l'infertilité

Tableau 9 : Analyse des facteurs associés à la prématurité (modèle logistique complet) ...

Tableau 10 : Odds Ratio de prématurité lié au contexte de la conception selon le modèle logistique et la population d'analyse

Tableau 11 : Analyse des facteurs associés à un petit poids de naissance (modèle logistique complet)

Tableau 12 : Odds Ratio de petit poids de naissance lié au contexte de la conception selon le modèle logistique et la population d'analyse

Tableau 13 : Odds Ratio de prématurité lié au type de traitement de l'infertilité selon le modèle logistique et la population d'analyse

Tableau 14: Odds Ratio de petit poids de naissance lié au type de traitement de l'infertilité selon le modèle logistique et la population d'analyse

Tableau 15 : Caractéristiques sociodémographiques des différents groupes infertiles considérés 


\section{LISTE DES FIGURES}

Figure 1: Part des enfants nés après AMP en 2011 selon le type d'AMP et l'origine des gamètes

Figure 2: Sélection de la population d'étude

Figure 3 : Décomposition de l'effet total du traitement de l'infertilité sur le risque de prématurité et de petit poids de naissance en un effet direct et un effet indirect médié par la gémellité et la prématurité

Figure 4: Pondération de l'enquête Elfe en maternité 


\section{INTRODUCTION}

Depuis les premiers succès de la fécondation in vitro (FIV) il y a une trentaine d'années (Louise Brown en 1978 au Royaume-Uni, Amandine en 1982 en France), le recours aux techniques d'assistance médicale à la procréation (AMP) n'a cessé de croître dans les pays développés. Ainsi, en France métropolitaine, la FIV ne représentait que 0,52\% des naissances en 1988 [1], contre 2,3\% en 2010 [2]. Plus généralement, 5,6\% des enfants ont été conçus grâce à un traitement médical de l'infertilité en 2010 selon l'enquête nationale périnatale [2] : 2,3\% suite à une FIV , 1,0 \% suite à une insémination artificielle et 2,3\% suite à une induction de l'ovulation seule, soit environ 45000 naissances par an en France.

De nombreuses questions se posent encore sur les conséquences des techniques d'AMP sur la santé et le devenir des enfants à court et à long terme [3], au-delà du taux très élevé de naissances multiples observé quel que soit le traitement, et de l'élévation de la prématurité qui en découle. L'innocuité de l'AMP fait l'objet de controverses dans la littérature scientifique, du fait notamment de la difficulté de construire des études permettant d'analyser le lien entre un facteur d'exposition pluriel (AMP) et un facteur à expliquer complexe (état de santé des enfants), tout en tenant compte des nombreux facteurs de confusion connus (développement fœtal multiple, âge maternel, parité, niveau socio-économique...). Les études comparant les enfants conçus avec une aide médicale aux enfants conçus naturellement peuvent ainsi aboutir à comparer des enfants plus souvent issus de grossesse multiple et plus souvent prématurés à des enfants plutôt singletons (issus de grossesse unique) et à terme. Pour cette raison, les auteurs se restreignent parfois à l'étude des singletons, voire aux singletons nés à terme, mais se heurtent

à de nombreuses limites méthodologiques (faiblesse des effectifs, définition imprécise des critères évalués...). 
Quelques chercheurs se sont intéressés aux événements de santé sévères mais rares et ont mis en évidence une élévation du risque de malformations congénitales [4-7] ou de cancer [810], tandis que d'autres études de cohorte plus rassurantes ne montrent pas de risque accru de cancer après AMP [11]. Peu d'études ont porté sur le développement psychomoteur et pondéral, malgré l'élévation connue de certains risques (prématurité, petit poids de naissance), et là encore leurs résultats sont contradictoires $[3,12-15]$; il en va de même pour les risques de pathologies médicales ou chirurgicales et les risques épigénétiques [3, 16, 17].

Si l'AMP semble associée à une augmentation de risques pour la santé des enfants, il n'existe pas d'arguments actuellement pour un lien causal avec l'intervention médicale ellemême. Il est difficile de distinguer la part d'effet liée aux techniques de prise en charge utilisées (traitement hormonal, manipulation de gamètes) de celle liée à l'infertilité des couples (c'est-àdire aux caractéristiques phénotypiques et génétiques propres des parents, souvent plus âgés, avec une gamétogenèse parfois imparfaite) [18]. Certains auteurs ont suggéré que l'infertilité per se, en dehors de tout traitement, pourrait être un facteur de risque de complications obstétricales et périnatales [19-23]. Dans une étude de cohorte norvégienne, Romundstad et al ont comparé les taux de petit poids pour l'âge gestationnel, de prématurité et de mortalité périnatale chez des singletons dont la mère avait conçu au moins un enfant spontanément et un autre avec une aide médicale, sans observer de différence parmi ces enfants ; ils concluaient de leurs résultats que les surrisques décrits par rapport à la population générale pouvaient être attribuables aux déterminants de l'infertilité plutôt qu'aux traitements [24]. Une piste intéressante pour démêler ces effets est de constituer deux groupes témoins de comparaison d'enfants conçus naturellement, un dont les parents ont eu des difficultés pour les concevoir, et un dont les parents n'ont pas eu de problème d'infertilité [25-29]. En cas d'effets indépendants du traitement et de l'infertilité, une augmentation du risque est attendue entre les trois groupes (hypothèse d'un "effet dose" croissant allant des parents fertiles aux infertiles modérés non traités aux infertiles traités). La constitution des deux groupes non traités soulève néanmoins 
des problèmes complexes pour les comparaisons ultérieures car les parents recourant aux traitements d'AMP pourraient être caractérisés par des situations socio-économiques plus favorables que la population moyenne [30, 31], même dans les pays qui couvrent les frais de traitement comme la France [32]. Or la santé et le développement des enfants sont très marqués par les inégalités sociales [33]. Ce facteur pourrait donc avoir une forte influence et moduler voire "contrebalancer" l'effet négatif des facteurs liés à l'infertilité des parents et aux traitements médicaux de l'infertilité, notamment la prématurité.

Nos hypothèses de travail sont ainsi que les enfants conçus avec une aide médicale pourraient avoir (toutes choses étant égales par ailleurs) un développement et un état de santé :

- moins favorables du fait du traitement de l'infertilité, de l'infertilité des parents, du taux élevé de grossesses multiples - ces trois facteurs pouvant contribuer par exemple à une plus grande prématurité ;

- plus favorables du fait d'une meilleure situation socio-économique familiale, avec l'hypothèse d'un effet positif des facteurs sociaux contrebalançant (partiellement ?) l'effet négatif des facteurs médicaux.

Il existe très peu de données publiées jusqu'à présent sur les enfants français issus d'AMP, ou conçus grâce à une stimulation ovarienne simple. L'objectif de ce travail est d'utiliser les données de la cohorte Elfe (Étude Longitudinale Française depuis l'Enfance) pour étudier l'état de santé des enfants conçus avec une aide médicale [34]. La description précise des caractéristiques (sociodémographiques entre autres) des familles y ayant eu recours devrait permettre d'explorer les facteurs jouant un rôle sur la santé de ces enfants et de faire la part des conséquences propres au traitement. Une approche longitudinale est un atout important pour l'étude des enfants conçus avec une aide médicale, la collecte prospective des données permettant en effet de s'assurer que les facteurs de l'environnement entourant la naissance (traitement de l'infertilité, facteurs socio-économiques et médicaux) sont bien enregistrés 
indépendamment de la survenue des événements étudiés. Dans le cadre de cette thèse n'ont été analysés que des indicateurs de début de vie (0 et 2 mois). À plus long terme, l'ambition est d'étudier le devenir des enfants conçus avec une aide médicale, en particulier leur développement psychomoteur et pondéral entre la naissance et 3 ans. 


\section{CONTEXTE MÉDICAL}

\subsection{INFERTILITÉ}

\section{Définition}

Le vocabulaire de l'infertilité est complexe [35]. Différentes notions peuvent en effet être distinguées: inaptitude à la fécondation (obtention d'une grossesse) et inaptitude à la procréation (obtention d'une naissance vivante), absence de grossesse ou de naissance volontaire ou involontaire, ces notions étant considérées soit au niveau individuel, soit au niveau du couple. L'infertilité est en théorie un état, qui existe indépendamment des tentatives de conception, mais qui ne peut être révélé que par celles-ci. Il est de ce fait difficile de caractériser cette infertilité et d'en mesurer la prévalence dans une population. Une approche fréquente est d'estimer l'infécondité (l'absence de grossesse ou de naissance), elle-même indicateur de l'infertilité (l'inaptitude à l'obtention d'une grossesse ou d'une naissance) [36], par exemple en recueillant des données sur le délai qui a été nécessaire pour concevoir. Cette information permet de définir selon la longueur du délai des "degrés d'infertilité", mais, obtenue a posteriori, elle exclut par construction les situations de stérilité totale ou de très faible fertilité : elle concerne plutôt des situations d'hypofertilité (couples ayant des chances réduites mais non nulles d'obtenir une grossesse). Une autre approche consiste à interroger les couples sur la durée d'exposition au risque de conception sans qu'il en ait résulté de grossesse, l'exposition étant définie par l'existence de relations sexuelles et l'absence de contraception [37]. Il est également possible, au moyen d'enquêtes, de recueillir rétrospectivement les difficultés de conception perçues par les couples, ou de s'informer sur les consultations médicales et les traitements suivis. Aucune de ces méthodes n'est totalement satisfaisante, en particulier elles permettent rarement de prendre en compte l'hétérogénéité de 
l'exposition (variable en fonction du comportement sexuel des couples, notamment la fréquence et le moment des rapports).

Nous nous restreindrons ici à la difficulté pour un couple de concevoir un enfant (c'est-àdire mener à terme une grossesse) alors qu'il se trouve "exposé au risque" de conception en ayant des rapports sexuels non protégés ; un couple sera considéré comme infertile s'il n'a pas pu concevoir après 12 mois de tentative. Selon l'Observatoire épidémiologique de la fertilité en France (Obseff) 2007-2008 [38], 24 \% des couples restent sans enfant après 12 mois de tentative sans contraception, cette proportion étant encore de $11 \%$ après 24 mois. L'ENP 2003 estimait quant à elle la fréquence de l'infécondité involontaire après 12 mois sans contraception à 18\%, et à $8 \%$ après 24 mois [39]. Ces deux études françaises reposaient sur des méthodologies très différentes. L'Obseff mettait en œuvre l'approche transversale des tentatives en cours (un échantillon aléatoire de femmes n'utilisant pas de contraception et ayant des rapports sexuels réguliers était interrogé sur le délai écoulé depuis le début de cette période sans contraception). L'ENP est une enquête rétrospective portant sur des femmes venant d'accoucher, et exclut de fait les couples restant sans enfant, ce qui tend à sous-estimer le taux d'infécondité involontaire.

\section{Étiologies}

Après un examen clinique, un bilan hormonal de la femme, une évaluation de la perméabilité tubaire et un spermogramme, le diagnostic étiologique est le plus souvent celui d'une infertilité d'origine mixte, liée à des troubles concernant les deux membres du couple, dans $40 \%$ des cas [40]. Une étiologie féminine isolée est identifiée dans 30 à $40 \%$ des cas, une étiologie masculine isolée dans 10 à $20 \%$ des cas, tandis que l'infertilité reste inexpliquée dans environ $15 \%$ des cas [40]. 
- Causes d'infertilité féminine [37]

Les principales causes d'infertilité féminine peuvent être classées en troubles de l'ovulation et causes mécaniques.

Les troubles de l'ovulation sont généralement d'origine endocrinienne. Le syndrome des ovaires polykystiques (SOPK) en est la principale cause; les étiologies hypothalamohypophysaires (atteinte de l'axe des gonadotrophines FSH et LH conduisant à un défaut de commande ovarienne), l'insuffisance ovarienne prématurée et les hyperandrogénies autres que le SOPK sont plus rarement retrouvées. Le SOPK est une pathologie d'origine ovarienne touchant environ $10 \%$ des femmes. Il résulte d'une altération de la sélection du follicule dominant avec une accumulation de petits follicules qui sécrètent des androgènes en excès. L'insuffisance ovarienne prématurée est une forme accélérée de perte folliculaire avec une aménorrhée survenant avant l'âge de 40 ans, pouvant être de cause génétique (anomalies du chromosome $\mathrm{X}$ ), auto-immune ou toxique (après chimiothérapie, radiothérapie).

Les causes mécaniques et obstructives regroupent l'endométriose, la sténose tubaire bilatérale et les causes utérines. L'endométriose touche environ 5 à $10 \%$ des femmes. Elle est liée à l'implantation de tissu endométrial en dehors de la cavité utérine (dans la cavité péritonéale, sur les ovaires), engendrant une réponse inflammatoire et la synthèse de néovaisseaux. Les mécanismes de cette pathologie sont mal connus. L'endométriose se manifeste cliniquement par des douleurs au moment des règles et des rapports sexuels. Elle induit souvent une anomalie du stock de follicules ovariens et des troubles de l'implantation embryonnaire. La sténose tubaire bilatérale est le plus souvent la conséquence d'une infection bactérienne sexuellement transmissible de type Chlamydia trachomatis, plus rarement d'une tuberculose génitale. Les causes utérines d'infertilité les plus fréquentes sont les malformations utérines (cloison utérine, exceptionnellement l'absence d'utérus), les synéchies utérines, et surtout les polypes de l'endomètre et les fibromes utérins. 
- Causes d'infertilité masculine [37]

L'exploration de l'infertilité masculine repose avant tout sur l'analyse du sperme, grâce à un spermogramme évaluant le volume du sperme d'un éjaculat, le nombre total de spermatozoïdes, la concentration, la mobilité, la vitalité et la morphologie spermatiques. L'infertilité masculine peut ainsi être classée selon trois types de situations : un trouble de la formation et de la production des spermatozoïdes par le testicule (insuffisance testiculaire), une anomalie post-testiculaire qui peut se manifester par des lésions des voies génitales ou une absence de maturation des spermatozoïdes (pathologie post-testiculaire), ou une perturbation de la fonction sexuelle empêchant le sperme d'accéder aux voies génitales féminines (dysfonction sexuelle).

L'insuffisance testiculaire est la cause la plus fréquente d'infertilité masculine. L'altération de la spermatogénèse peut porter sur le nombre et/ou la mobilité et/ou la morphologie et/ou la fonction des spermatozoïdes, caractérisant par exemple une oligo-asthéno-térato-spermie (OATS) voire à l'extrême une azoospermie sécrétoire. Le déficit de fabrication des spermatozoïdes est soit constitutionnel (anomalies des chromosomes sexuels, translocations ou inversions de chromosomes autosomiques, mutations de gènes autosomiques...), soit acquis, lié à une cryptorchidie (absence d'un ou des deux testicules dans le scrotum, en raison d'un arrêt de la migration testiculaire lors de son trajet de descente), un varicocèle (dilatation des veines spermatiques), un hypogonadisme hypogonadotrope (la baisse des gonadotrophines FSH et LH altèrant la commande gonadique testiculaire), une orchite, un traumatisme, une torsion testiculaire, un traitement par chimiothérapie ou radiothérapie... Dans près de $50 \%$ des cas, aucune cause n'est retrouvée.

Les pathologies post-testiculaires regroupent les troubles de la maturation des spermatozoïdes pendant le transit épididymaire et les lésions obstructives des voies génitales masculines, qui empêchent le mélange des spermatozoïdes au liquide séminal lors de 
l'éjaculation (aboutissant à une azoospermie excrétoire). L'obstruction peut être constitutionnelle (liée par exemple à une agénésie des canaux déférents) ou consécutive à une infection, un traumatisme ou une intervention chirurgicale.

Les dysfonctions sexuelles peuvent être une cause d'infertilité masculine si elles empêchent l'éjaculation : le sperme n'atteint pas les voies génitales féminines. Les troubles de l'érection et de l'éjaculation sont d'origine psychogène ou neurologique (lésions médullaires responsables d'anajéculation ou d'éjaculation rétrograde par exemple).

\section{Une infertilité de plus en plus fréquente?}

L'infertilité serait un phénomène en hausse, du fait notamment du recul de l'âge des couples désirant concevoir un premier enfant [41]. En effet, la fertilité féminine diminue avec l'âge et chute après 35 ans [42] : l'insuffisance ovarienne débutante est la première cause d'infertilité féminine après l'âge de 35 ans [37]. Elle se définit par une diminution physiologique du nombre de follicules ovariens, qui s'accompagne le plus souvent d'une altération de la qualité ovocytaire, entraînant une augmentation du taux de fausses couches spontanées et d'anomalies chromosomiques fœtales [43]. Cette situation répond mal aux traitements de stimulation ovarienne ou d'assistance médicale à la procréation du fait de la faible réserve folliculaire, la quantité et la qualité des ovocytes disponibles pour une fécondation faisant par conséquent elles aussi défaut. Il n'existe pas de traitement susceptible d'améliorer la production de follicules ovariens. La fertilité masculine diminue également après 40 ans [44], phénomène lui aussi à l'origine d'une augmentation du risque de mort fœetale [43, 45]. 
Il existerait par ailleurs une tendance à la baisse de qualité du sperme chez les hommes [46-49], liée à des causes génétiques ou constitutionnelles (augmentation des taux de cancer du testicule [50-56], et de malformations du type cryptorchidie et hypospadias [57-60]), et probablement aussi à des facteurs environnementaux (surpoids, tabagisme, exposition à certains polluants organiques persistants et métaux lourds, aux phtalates) [61]. 


\subsection{TRAITEMENTS DE L'INFERTILITÉ}

Différentes interventions médicales plus ou moins invasives peuvent être proposées aux couples infertiles [62].

\section{Stimulation ovarienne}

La stimulation ovarienne est une induction simple de l'ovulation par traitement hormonal, sans manipulation de gamètes, qui n'entre pas dans le cadre de l'AMP et n'est soumise qu'à des règles de bonnes pratiques. Elle permet, grâce à un suivi échographique et sanguin, de recruter des follicules matures, de prédire le jour de l'ovulation et de synchroniser la rencontre des gamètes, tout en s'assurant qu'il n'y a pas d'hyperstimulation, situation plus à risque de grossesse multiple (l'objectif est d'obtenir un à trois follicules matures fécondables).

La stimulation ovarienne simple nécessite que le couple ait des rapports sexuels.

Les indications sont les troubles de l'ovulation.

\section{Assistance médicale à la procréation}

En France, le terme d'assistance médicale à la procréation (AMP) regroupe les techniques consistant à manipuler des gamètes pour obtenir une fécondation, ou à manipuler l'embryon. Elles sont strictement encadrées par la loi et suivies par l'Agence de la Biomédecine (articles L. 2141-1 à L. 2141-4 du code de la santé publique). Leur objet est de "remédier à l'infertilité médicalement reconnue d'un couple ou d'éviter la transmission à l'enfant ou à un membre du couple d'une maladie d'une particulière gravité", "l'homme et la femme formant le couple [devant] être vivants, en âge de procréer et consentir préalablement au transfert des embryons ou à l'insémination" [63]. L'AMP est aussi indiquée à titre préventif avant des traitements stérilisants.

Le don de gamète ou d'embryon repose sur l'anonymat, le volontariat et la gratuité. 
La définition de l'AMP retenue par l'OMS diffère [64] et ne considère que les techniques impliquant une manipulation in vitro de gamètes ou d'embryons dans le but d'obtenir une grossesse. Elle n'inclut pas l'insémination articificielle, contrairement à la définition française.

\section{L'insémination artificielle (IA)}

Il s'agit de la technique d'AMP la plus simple. Le sperme, du conjoint pour une IAC (insémination artificielle avec spermatozoïdes du conjoint), ou d'un donneur pour une IAD (insémination artificielle avec spermatozoïdes de donneur), est préparé puis déposé dans l'utérus (IA intra-utérine) ou au niveau du col de l'utérus (IA intra-cervicale) de la femme, de façon synchronisée avec l'ovulation déclenchée par stimulation. La fécondation se fait naturellement, in vivo.

Le taux de grossesse en France après insémination artificielle était de 12,3\% par tentative en $2011[65]^{1}$, année de naissance des enfants de la cohorte Elfe. Ce taux était de 12,9\% en 2012 (d'après le dernier rapport d'activité publié par l'ABM) [66].

L'IAC est indiquée en cas d'altérations de la glaire cervicale, d'altérations modérées du sperme ou d'infertilité inexpliquée (en première intention ou après échec de la stimulation simple) ; l'IAD en cas d'azoospermie sécrétoire ou de maladie génétique grave du conjoint.

\section{La fécondation in vitro (FIV)}

Cette technique plus invasive reproduit en laboratoire les premières étapes du développement de l'embryon, de la fécondation aux premières divisions. Après une forte stimulation hormonale suivie d'une ponction folliculaire, les gamètes des deux conjoints ou de

\footnotetext{
${ }^{1}$ Les données d'activité d'AMP recueillies par l'ABM au 31 décembre de chaque année concernent toujours l'année antérieure afin de pouvoir établir le lien entre les tentatives et les naissances. Les statistiques présentées à une année $n$ concernent donc des tentatives réalisées lors de l'année n-2 et des naissances survenues principalement lors de l'année n-1, du fait du délai nécessaire pour croiser les résultats, les valider et les éditer.
} 
donneur(s) sont préparés puis mis en fécondation in vitro par mise en contact de chaque ovocyte avec un nombre suffisant de spermatozoïdes. Les embryons obtenus sont ensuite transférés dans l'utérus de la femme sous contrôle échographique. Le nombre d'embryons transférés dépend de l'âge de la femme et des centres d'AMP, il tend à diminuer pour réduire le nombre des grossesses multiples et leurs complications (en 2010, un embryon unique était transféré dans $33,4 \%$ des cas, deux embryons dans $57,4 \%$ des cas et trois embryons ou plus dans $8,5 \%$ des cas ; ces proportions étaient de 36,5\%, 55,9\% et 7,6\% respectivement en 2011 [66]).

Le taux de grossesse en France après FIV était de 22,9 \% par tentative en 2011 [65]. Il était de $23,8 \%$ en 2012 [66].

La FIV est indiquée en cas d'infertilité tubaire ou d'anomalies du sperme, en cas d'infertilité inexpliquée ou après échec des IA.

\section{La FIV avec micro-injection (FIV-ICSI, intracytoplasmic sperm injection)}

L'ICSI consiste à injecter un spermatozoïde dans le cytoplasme de chaque ovocyte. Les autres étapes sont identiques à celles de la FIV.

Le taux de grossesse en France après ICSI était de 24,4 \% par tentative en 2011 [65]. Il était de $24,0 \%$ en 2012 [66].

L'ICSI a permis la prise en charge d'une grande partie des problèmes d'infertilité masculine [67]. Elle est indiquée devant une anomalie majeure du sperme ou une azoospermie excrétoire, et en cas d'échec de la FIV classique. Elle représente cependant désormais $64 \%$ des FIV [65], ce qui semble indiquer un recours plus large. 


\section{Le transfert d'embryons congelés (TEC)}

Si le couple y consent, les embryons surnuméraires obtenus par FIV sont congelés et pourront être utilisés ultérieurement pour un nouveau transfert embryonnaire : c'est le transfert d'embryons congelés (TEC). Un couple stérile ou à risque de transmission de maladie génétique peut demander à recevoir un embryon congelé issu d'un autre couple.

Le taux de grossesse en France après TEC était de 17,6 \% par tentative en 2011 [65]. Il était de $18,7 \%$ en 2012 [66].

\section{Quelques chiffres}

En 2011 en France, l'activité d'AMP a représenté 141277 tentatives, avec 41,8\% d'inséminations et 58,2\% de FIV (classiques / ICSI / TEC) [65]. Dans $5 \%$ des cas, les tentatives font appel à un don. Les taux de naissance par tentative intraconjugale (réalisée avec les gamètes des deux membres du couple) varient de $9,5 \%$ après insémination à $20,1 \%$ après ICSI.

Ainsi, 23127 enfants sont nés après une AMP en 2011, soit 2,8 \% des 823394 enfants nés de la population générale [67]. Parmi ces naissances, $94 \%$ sont issues d'une AMP intraconjugale (24,7 \% par insémination, 37,6 \% par ICSI, 19,6 \% par FIV classique, 12,3\% par TEC), tandis que $6 \%$ des enfants sont nés grâce à un don : de spermatozoïde $(4,8 \%)$, d'ovocyte $(0,9 \%)$, ou d'embryon ( $0,1 \%$ soit 23 naissances). La figure 1 illustre ces différentes proportions. 
Figure 1 : Part des enfants nés après AMP en 2011 selon le type d'AMP et l'origine des gamètes $(\mathrm{N}=23$ 127)

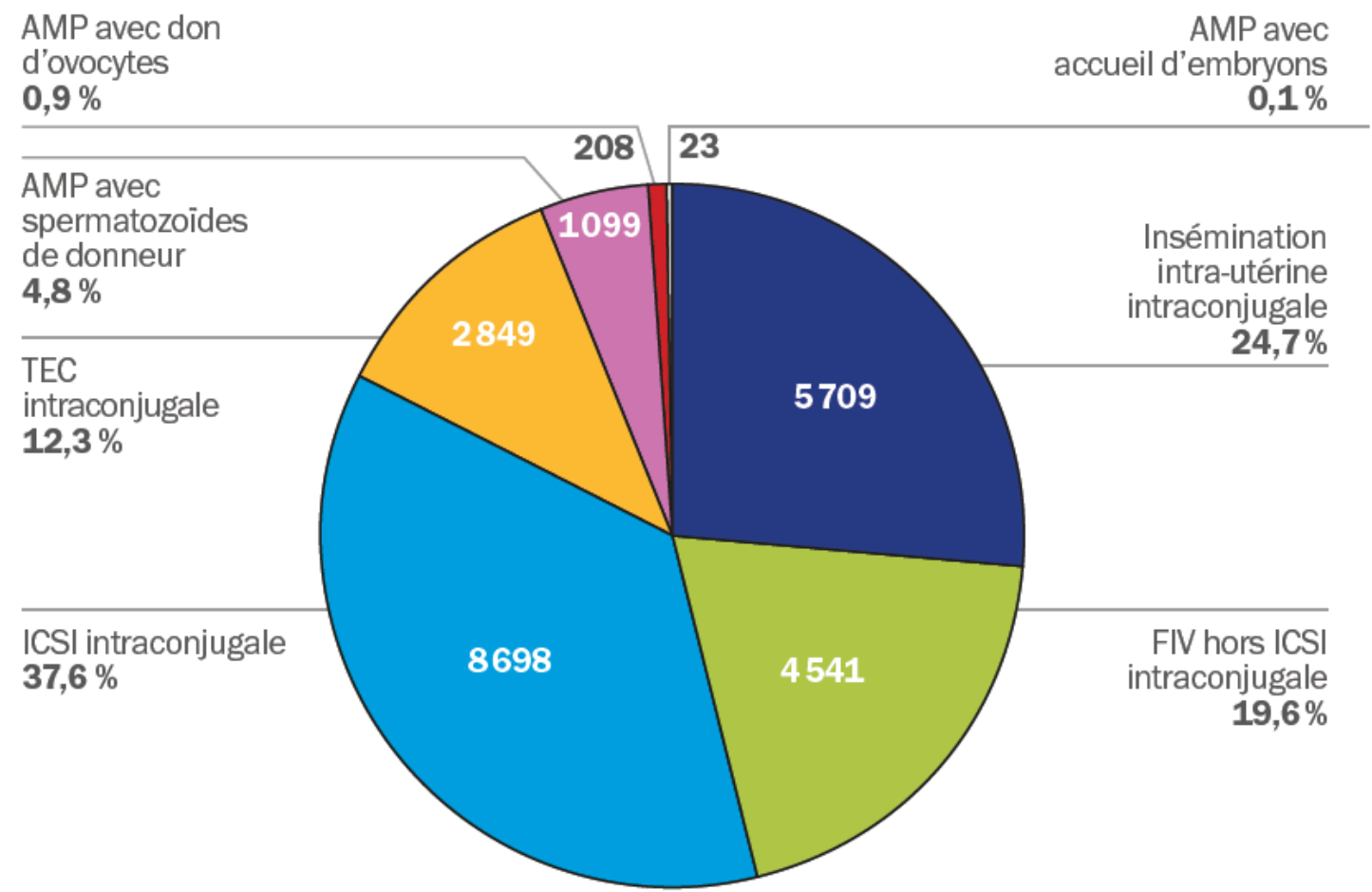

Source : Rapport d'activité annuel d'AMP 2011, Agence de la biomédecine, 2012. 


\subsection{COMPLICATIONS ASSOCIÉES AUX TRAITEMENTS DE L'INFERTILITÉ}

\section{Complications de la grossesse et complications néonatales}

\section{Grossesses multiples}

Le risque principal lié aux traitements de l'infertilité est la survenue d'une grossesse multiple et ses complications.

L'ensemble des complications néonatales après AMP ont longtemps été attribuées à ce seul facteur, même si récemment plusieurs auteurs ont suggéré d'autres causes possibles, liées aux caractéristiques des couples traités ou au traitement lui-même [3].

Quelle que soit la méthode utilisée, une forte proportion de grossesses multiples est observée. En France en 2010, l'enquête nationale périnatale a relevé un taux d'accouchement multiple de $19 \%$ après traitement médical contre $1,7 \%$ dans la population générale [2]. Ce taux est de 17,6 \% en FIV et de 18,7 \% en ICSI d'après l'agence de biomédecine, malgré une politique de transfert limitée à un ou deux embryons depuis quelques années. En Europe, ce taux était en moyenne de 20,6\% en 2010 [69] et variait fortement selon la stratégie des pays en matière de nombre d'embryons transférés, allant de 5,9\% en Suède à 38,0 \% en Bulgarie.

Chez l'enfant, le développement fœtal multiple entraîne entre autres une élévation du taux de prématurité et de petit poids à la naissance, et est à l'origine d'une morbi-mortalité néonatale plus importante $[3,70,71]$. Il existe en outre des risques pour la santé maternelle.

\section{Prématurité et petit poids de naissance}

La prématurité correspond à une naissance survenant avant 37 semaines d'aménorrhée (SA). Elle est subdivisée en prématurité moyenne entre 33 et 37 SA, grande prématurité entre 28 et 33 SA (environ $10 \%$ des cas de prématurité) et très grande prématurité avant 28 SA (moins de $5 \%$ des cas). Les conséquences de la prématurité sont importantes sur 
la morbi-mortalité néonatale, avec comme déterminant principal l'âge gestationnel : l'étude française Épipage rapportait en 1997 une survie de $31 \%$ à $24 \mathrm{SA}, 78 \%$ à 28 SA et $97 \%$ à 32 SA [71]. Le risque de mortalité des enfants de prématurité moyenne (entre 33 et 37 SA), bien que très inférieur à celui d'un grand prématuré, est toutefois 3 à 5 fois supérieur à celui d'un nouveau-né à terme [72]. La survie des prématurés s'est nettement améliorée depuis la fin des années 1990 avec la mise en place d'une politique de régionalisation des soins et l'organisation des soins périnatals (des niveaux de soins ont été définis pour les maternités en fonction de leurs capacités de prise en charge des nouveaux-nés).

La grossesse multiple est le principal facteur de risque de prématurité spontanée ; il en existe cependant de nombreux autres [72], parmi lesquels : un âge maternel extrême, un bas niveau socio-économique, le tabagisme, un faible intervalle intergénésique, le diabète gestationnel, les malformations de l'appareil génital maternel (dont certaines responsables d'infertilité). Il faut y ajouter les causes de prématurité médicalement induite (c'est-à-dire suivant une césarienne en dehors du travail ou un déclenchement du travail, sur décision médicale, en raison de risques pour la mère et l'enfant, le plus souvent dans un contexte d'hypertension artérielle sévère, de retard de croissance grave ou d'hémorragie maternelle), en augmentation et qui concerne 35 à $40 \%$ des naissances prématurées [72, 73].

Le petit poids de naissance ou hypotrophie est défini par un poids de naissance inférieur à 2500 grammes. Il est bien entendu très lié à la prématurité, mais ces deux phénomènes ne se recouvrent pas totalement. Certaines hypotrophies résultent d'un retard de croissance intrautérin, dont les étiologies sont nombreuses (malformation utérine, tabagisme, alcoolisme, âge maternel extrême, primiparité, bas niveau socio-économique, pathologie fœtale, insuffisance placentaire par exemple), parmi lesquelles la grossesse multiple [74].

La prématurité concernait $18 \%$ des accouchements après AMP (24\% des enfants conçus) en France en 2010 contre 6,5\% en population générale [2]. Cependant, le taux de 
naissances multiples n'explique pas l'ensemble du phénomène de prématurité des enfants conçus par AMP, la prématurité étant également plus fréquente lors d'un accouchement unique ( $8,8 \%$ après AMP contre 5,3\% en population générale) [2]. Se pose donc la question d'un effet du traitement lui-même. Plusieurs équipes ont ainsi rapporté un sur-risque de prématurité et d'hypotrophie chez les singletons conçus par AMP, même après correction de l'âge maternel et de la parité (les femmes bénéficiant d'une AMP sont plus âgées et plus souvent primipares [75]) $[3,76-79]$.

\section{Impact ultérieur sur la santé et le développement des enfants ?}

Le rôle de l'AMP est suspecté dans différentes pathologies, mais ces risques restent faibles et à préciser ; dans la plupart des cas, ces pathologies sont associées à la prématurité. La prématurité et l'hypotrophie ont en effet des conséquences connues sur le développement psychomoteur et staturo-pondéral des enfants, entraînant en particulier des difficultés de croissance et d'apprentissage [80, 81]. Là encore, la question se pose d'une part de risque intrinsèque du traitement d'AMP. Les études menées chez les enfants singletons nés à terme n'ont pas montré de différence significative entre AMP et conception naturelle [12-15], mais ce point est difficile à évaluer, les enfants nés après prise en charge de l'infertilité bénéficiant sans doute d'un environnement socio-économique plus favorable pouvant contrebalancer (en partie) un possible effet du traitement de l'infertilité des parents [30-32].

Les techniques employées (traitements hormonaux, processus de fécondation, culture et congélation) peuvent-elles induire un sur-risque de malformations congénitales, de cancer ou d'autres maladies chez les enfants ? Comme indiqué en introduction, des études ont mis en évidence une élévation de certains risques de santé, mais leurs résultats restent controversés [411]. Il a également été rapporté une augmentation de l'incidence de pathologies rares liées à des modifications de gènes soumis à empreinte parentale impliqués dans le développement 
embryonnaire et fœtal $[82,83]$. Le mécanisme de l'apparition de ces maladies épigénétiques après AMP pourrait être lié au stress cellulaire entraîné par la manipulation des gamètes et des embryons in vitro [84]. Enfin, dans le cas de la FIV-ICSI, le risque de transmission de pathologies responsables d'infertilité masculine fait encore l'objet d'interrogations: les conséquences à long terme chez les enfants nés de cette technique relativement récente (première naissance en 1992) restent mal connues, les premières études de cohorte sont pour le moment rassurantes [85]. 



\section{OBJECTIF DE L'ÉTUDE}

\section{Objectifs principaux}

L'objectif de ce travail est d'étudier la santé des enfants conçus avec une aide médicale, selon deux grands axes :

1. Décrire les conditions entourant leur naissance, en particulier leur situation socioéconomique et leur état de santé dans les deux premiers mois de vie.

2. Explorer les facteurs impactant sur leur santé, en particulier, faire la part du traitement de l'infertilité et des autres facteurs dans les complications pouvant survenir après une conception obtenue avec une aide médicale.

\section{Objectif secondaire}

Un objectif secondaire est d'étudier plus spécifiquement et de manière isolée les sousgroupes "fécondation in vitro" et "stimulation ovarienne seule" lorsque les effectifs le permettent. 



\section{MATÉRIELS ET MÉTHODES}

\subsection{LA COHORTE ELFE}

L'étude est basée sur l'analyse des données de la cohorte Elfe (Étude Longitudinale Française depuis 1'Enfance) [86], première grande cohorte d'enfants nés en France métropolitaine, suivis depuis la naissance jusqu'à l'âge adulte. Après une phase pilote auprès de 500 familles en 2007, plus de 18000 enfants ont été recrutés en 2011.

L'objectif général de la cohorte Elfe est de suivre l'enfant et sa famille pendant une vingtaine d'années afin de comprendre comment l'environnement, de la période intra-utérine à l'adolescence, influence le développement, la santé et la socialisation de l'enfant [87]. Trois orientations majeures ont ainsi été définies : les sciences sociales, la santé et la santéenvironnement. Ces trois axes regroupent les nombreux facteurs à prendre en compte dans l'évaluation de l'environnement dans lequel évolue l'enfant, au niveau familial, social (scolaire et relationnel), économique ou géographique. Elfe devrait permettre de relever simultanément et de manière prospective ces différentes dimensions, dans une approche pluridisciplinaire, d'étudier leurs interactions, et de les mettre en relation avec les principaux aspects du devenir de l'enfant : le développement (cognititivo-moteur ou sensoriel, la croissance, la puberté), la santé (notamment la survenue de pathologies fréquentes : surpoids et obésité, asthme et autres manifestations allergiques, troubles mentaux et du comportement ; les accidents, la présence de handicaps), les acquisitions scolaires (lecture, écriture..), la socialisation et la qualité de vie.

Une collecte prospective de données est ainsi prévue auprès de différentes sources : la mère, le père, le carnet de santé, le dossier médical en maternité, le médecin de l'enfant, la 
sécurité sociale. Les informations recueillies durant les premièrs étapes de l'enquête portent sur : le mode de vie pendant la grossesse, le climat conjugal, les relations familiales, les conditions de vie (mode de garde, organisation de la vie quotidienne, caractéristiques du logement, exposition à des toxiques, agents physiques), les pratiques culturelles et les loisirs, l'alimentation de l'enfant, sa croissance, son développement et son tempérament, sa santé, sa scolarité, la santé des parents, les recours aux soins... Des collections biologiques sont également prévues (sang, colostrum, urines, méconium, selles, cheveux, ARN sanguin, ADN extrait des globules blancs maternels et de l'enfant ou extrait des cellules du cordon...), permettant l'évaluation d'expositions précoces toxiques ou nutritionnelles, ainsi que de marqueurs de l'état de santé (marqueurs métaboliques, d'inflammation, de stress oxydatif chez la mère et le fœtus ; marqueurs de contacts infectieux ; marqueurs génétiques de la mère et de l'enfant) [87].

Le tableau 1 rapporte les principales étapes du suivi (réalisées ou planifiées) pour les huit premières années de la cohorte Elfe. Le travail présenté ici s'appuie sur les données recueillies auprès de la mère en maternité (questionnaire réalisé en face-à-face et relevé du dossier médical de la mère et de l'enfant) et aux deux mois de l'enfant (enquête téléphonique). 
Tableau 1 : Principales étapes du suivi pour les huit premières années de la cohorte Elfe

\begin{tabular}{|c|c|}
\hline Âge de l'enfant (dates) & Modalités de suivi \\
\hline Naissance (avril - décembre 2011) & $\begin{array}{l}\text { - Entretien en face-à-face avec la mère } \\
\text { - Relevé du dossier médical obstétrical } \\
\text { - Prélèvements biologiques en salle } \\
\text { d'accouchement et en suites de couches } \\
\text { (sous-échantillon) }\end{array}$ \\
\hline 2 mois (juin 2011 - mars 2012) & $\begin{array}{l}\text { - Enquête téléphonique } \\
\text { - Envoi d'un piège à poussière au domicile } \\
\text { (sous-échantillon) }\end{array}$ \\
\hline 3 - 10 mois (juillet - novembre 2011) & - Questionnaires postaux de suivi alimentaire \\
\hline 1 an (2012) & - Enquête téléphonique \\
\hline 2 ans (2013) & $\begin{array}{l}\text { - Enquête téléphonique } \\
\text { - Autoquestionnaire postal au médecin }\end{array}$ \\
\hline 3 ans (2014) & $\begin{array}{l}\text { - Enquête à domicile sur un sous-échantillon : } \\
\text { - test cognitif } \\
\text { - prélèvements urine et selles } \\
\text { - piège à poussières } \\
\text { - Enquête téléphonique }\end{array}$ \\
\hline 4 ans $(2015)$ & $\begin{array}{l}\text { - Enquête internet: } \\
\text { - Questionnaires-jeux pour l'enfant sur } \\
\text { différents aspects de socialisation } \\
\text { - Budget-temps pour les parents }\end{array}$ \\
\hline 5 ans (2016) & - Enquête téléphonique \\
\hline 6 ans (2017) & $\begin{array}{l}\text { - Bilan des compétences scolaires (moyenne } \\
\text { section de maternelle) }\end{array}$ \\
\hline $8-9$ ans $(2019-2020)$ & $\begin{array}{l}\text { - Enquête téléphonique } \\
\text { - Bilan de santé comprenant : } \\
\text { - un examen médical } \\
\text { - des tests psychomoteurs } \\
\text { - des prélèvements urine et sang } \\
\text { - Bilan des compétences scolaires }\end{array}$ \\
\hline
\end{tabular}

Source : Présentation générale de l'étude Elfe, Plateforme d'accès aux données RE-CO-NAI

La population cible de la cohorte Elfe est l'ensemble des nourrissons nés en 2011 en France métropolitaine, à l'exception de certaines circonstances particulières difficilement 
compatibles avec l'enquête, pour des raisons pratiques de sécurité et d'efficacité [87]. Les familles n'ont ainsi pas été sollicitées en cas de parents mineurs ou non en mesure de donner un consentement éclairé, ni en cas de naissances multiples de plus de deux enfants (situation relativement rare, seuls 208 accouchements triples ou quadruples ayant été observés en 2011 [68], soit 1,5\% des accouchements multiples). Les grands prématurés (nés avant 33 semaines d'aménorrhée) n'ont pas non plus été retenus dans Elfe, bien qu'ils représentent 1,9\% des naissances en 2010 [2], soit 25,7\% de l'ensemble des prématurés. Ces enfants font en effet l'objet d'un suivi spécifique dans une autre cohorte lancée en 2011, Épipage 2 [88], à laquelle leurs parents pouvaient se voir proposer de participer.

Les critères d'inclusion de la cohorte Elfe sont ainsi [89] : un terme supérieur ou égal à 33 semaines d'aménorrhée ; une naissance simple ou gémellaire ; une mère majeure résidant en métropole. Les familles étrangères pouvaient participer à l'étude, à condition que la mère lise le français, l'anglais, l'arabe ou le turc, langues le plus souvent parlées par les mères étrangères accouchant en France et dans lesquelles les formulaires ont été traduits.

Le recrutement de la cohorte Elfe s'est effectué sur 25 jours répartis en quatre vagues représentant plusieurs saisons de l'année 2011 (du $1^{\mathrm{er}}$ au 4 avril, du 27 juin au 4 juillet, du 27 septembre au 4 octobre et enfin du 28 novembre au 5 décembre). L'enquête était prévue initialement sur 16 jours répartis en quatre vagues de quatre jours, prédéfinis de telle sorte que $48 \%$ des naissances d'enfants Elfe figurent également dans l'échantillon démographique permanent de l'Insee [89] ; elle a finalement été étendue à 25 jours pour compenser un recrutement plus faible qu'escompté.

L'inclusion des enfants s'est déroulée selon un plan de sondage à deux degrés : 344 maternités ont d'abord été tirées au sort parmi les 540 maternités métropolitaines françaises. L'étude a ensuite été proposée à toutes les mères accouchant dans les maternités sélectionnées. 
Le taux de participation a été de $93 \%$ pour les maternités tirées au sort et de $49 \%$ pour les mères éligibles. Au total, 18329 enfants ont été inclus, dont 288 paires de jumeaux. Des écarts régionaux de participation ont été observés, de ce fait l'échantillon ne respecte pas strictement la distribution régionale des naissances selon l'état-civil (l'Île-de-France et les Rhône-Alpes sont sous-représentées, tandis que le Nord-Pas-de-Calais est sur-représenté) [90].

Cette stratégie de recrutement a rendu nécessaire une pondération des données, tenant compte à la fois du plan de sondage et des biais de non-participation inhérents à ce type d'étude, afin d'assigner à chacun des nourrissons enquêtés un poids correspondant au nombre d'enfants qu'il représente dans la population cible et de présenter des résultats généralisables (ce point est repris et développé dans la partie plan d'analyse).

L'enquête en maternité, première phase de l'étude, a été réalisée essentiellement par des sages-femmes spécialement formées, chargées de l'information et du recueil du consentement des mères pendant leur séjour en maternité, puis du recueil des données [89]. Les enquêtrices ont saisi informatiquement :

- une fiche contact comprenant les coordonnées de la mère et du père,

- un questionnaire en face-à-face réalisé avec la mère, comportant des informations sur la situation sociodémographique de la mère et du père, le suivi de la grossesse, l'accouchement, la prise de médicaments pour des difficultés psychologiques, la consommation de tabac et d'alcool, l'organisation du suivi médical de l'enfant,

- des données extraites du dossier médical, qui concernaient le passé obstétrical de la mère, les pathologies antérieures, le bilan sanguin du $6^{\text {ème }}$ mois, les dépistages pendant la grossesse (toxoplasmose, CMV, hépatite $\mathrm{B}, \mathrm{VIH}$, syphilis) et les traitements en cas de dépistage positif, les complications de la grossesse (diabète gestationnel, hypertension artérielle, etc.), des précisions sur le suivi échographique (mesures 
anthropométriques du foetus, examens complémentaires, diagnostic anté-natal), sur l'accouchement et sur l'état de l'enfant à la naissance (score d'Apgar, etc.).

Les questionnaires « face-à-face » et « dossier médical obstétrique » ont été conçus à partir des questionnaires des enquêtes nationales périnatales afin de permettre des points de comparaison.

Les mères approchées qui ont refusé de participer à Elfe ont fait l'objet d'un recueil de données, anonyme et limité à quelques variables issues du premier certificat de santé établi dans les 8 jours suivant la naissance. Ces données ne sont pas encore accessibles et n'ont pas pu être exploitées ici [86].

Le questionnaire en face-à-face a été réalisé pour 99,3\% des mères ayant donné leur accord pour participer à l'étude Elfe, le relevé du dossier médical pour 99,0\% d'entre elles [90].

L'enquête aux deux mois de l'enfant est la première étape de suivi après l'inclusion des enfants Elfe en maternité [91]. Ce calendrier rapproché permettait de fidéliser les parents, en profitant de leur disponibilité puisque beaucoup étaient encore en congé maternité ou paternité peu de temps après l'accouchement, et de minimiser les biais de mémorisation pour les questions relatives à la grossesse à un moment encore proche de celle-ci. L'enquête s'est faite en quatre vagues, correspondant aux quatre vagues de l'enquête maternité.

Il s'agissait d'une enquête téléphonique réalisée par l'institut de sondage GFK. Un questionnaire catisé (informatisé en vue d'une enquête téléphonique) était administré à la mère, puis l'enquêteur annonçait à la fin de l'entretien qu'il allait contacter le père dans les jours suivants si des coordonnées étaient disponibles et si la mère ne s’y opposait pas ou n’indiquait pas que le père ne souhaitait pas participer. Le type de questionnaire posé aux parents était déterminé à partir de la situation familiale et de la personne avec qui vivait l'enfant : 
- questionnaires mère :

○ « référente » si l'enfant vivait avec elle tout le temps ou en alternance,

○ « non cohabitante » si l'enfant vivait exclusivement avec son père,

○ « référente enfant placé » si l'enfant ne vivait ni avec elle ni avec son père ;

- questionnaires père :

○ « référent » si l'enfant vivait exclusivement avec son père et si la mère avait répondu au questionnaire 'mère non cohabitante',

O « cohabitant » si la mère avait répondu à un questionnaire 'mère réfèrent' et que l'enfant vivait avec elle et son père ; il comporte des questions spécifiques sur la santé du père et ses rapport à l'enfant, mais aussi des questions déjà posées pour lesquelles il est nécessaire d'avoir les réponses du couple,

0 «non cohabitant» si la mère avait répondu à un questionnaire 'mère réfèrent' et que l'enfant vivait avec elle, exclusivement ou en garde alternée, ○ « cohabitant enfant placé », « non cohabitant enfant placé ».

Les objectifs principaux étaient de préciser les caractéristiques sociodémographiques de la famille de l'enfant, le lien avec l'enfant de chacune des personnes vivant avec lui, les caractéristiques du logement dans lequel vit l'enfant, et d'obtenir des premiers éléments sur son développement et sa santé. C'est également dans ce questionnaire deux mois qu'étaient abordées les questions relatives à l'histoire de la conception de l'enfant (grossesse désirée, contraception antérieure, temps nécessaire à l'obtention de la grossesse, antécédent de fausse couche pendant la période de tentative, consultation médicale dans le but de rendre la grossesse possible, conception obtenue grâce à un traitement, type de traitement utilisé).

Les données utilisées dans le cadre de ce travail sont celles issues du questionnaire «mère référente », avec un croisement des réponses du questionnaire «père cohabitant» pour vérification de certaines variables. 
L'enquête deux mois a pu être menée auprès de 90,4 \% des familles. Les mères de 16431 enfants $(89,6 \%)$ ont répondu au moins partiellement au questionnaire «mère référente ». Au total, parmi les questionnaires débutés, 15649 questionnaires «parent référent » ont été complètement documentés (15 540 questionnaires mère et 109 questionnaires père) [92]. 


\subsection{POPULATION D'ÉTUDE : approche avec trois groupes d'étude}

Seuls les enfants issus d'une grossesse désirée par les deux parents ont été étudiés ici, ceci pour rester dans le cadre de l'infécondité involontaire et éliminer le "bruit" des grossesses non planifiées. Par ailleurs, dans le cas d'une naissance gémellaire, un seul jumeau par paire a été retenu dans la population d'étude, par tirage au sort, afin de ne pas être confronté à des observations corrélées (le nombre de jumeaux était trop faible pour mettre en œuvre des méthodes spécifiques).

Vérifier l'hypothèse d'un effet propre du traitement d'AMP, indépendant notamment de l'infertilité des parents, nécessite de séparer effet du traitement et effet des caractéristiques de la population traitée. Pour cela, deux groupes témoins de comparaison d'enfants conçus naturellement (avec ou sans difficultés) ont été constitués dans ce travail. À partir du questionnaire de la mère réalisé aux deux mois de l'enfant, trois groupes d'étude ont ainsi été définis selon le contexte de la conception (figure 2) :

- le "groupe traitement" : enfants nés grâce à une "conception obtenue suite à un traitement", avec une restriction aux FIV avec ou sans ICSI, inséminations artificielles et stimulations ovariennes seules, les autres traitements (chirurgie, traitement du conjoint) sortant du champ de l'étude. Les TEC ne sont pas mentionnés explicitement dans le questionnaire, nous avons considéré qu'ils étaient inclus dans les FIV, bien qu'une catégorie "autre traitement" ait été proposée aux mères (elle représente 139 naissances).

- le "groupe infertile" : enfants conçus naturellement mais après une période d'infécondité, ("temps passé à essayer d'être enceinte" $\geq 1$ an). L'hypothèse est que les parents de ce groupe présentent des caractéristiques proches de celles des parents du groupe traité.

- le "groupe fertile" : enfants conçus naturellement sans période d'infécondité ("temps passé à essayer d'être enceinte" $<1 \mathrm{an})$. 
Soulignons ici l'importance d'une définition précise du groupe infertile, pour une comparabilité la plus grande possible avec le groupe traité. La spécification du groupe infertile soulève pourtant des difficultés : le délai de conception n'est pas un critère suffisant pour définir une infécondité involontaire, il faut également une notion "d'exposition au risque de grossesse". Or, si la mère a été interrogée dans Elfe sur ses habitudes contraceptives (type de méthode utilisée et moment de son arrêt), il n'y a pas eu en revanche de question portant sur la fréquence des rapports du couple ou l'existence de précautions anticonceptionnelles "naturelles", ni même sur l'ancienneté du couple ou sa stabilité.

Le risque est donc de regrouper dans un même groupe deux populations distinctes : des femmes infertiles avec un projet d'enfant et des femmes non planificatrices, qui ne prennent pas de contraception mais ne sont pas opposées à l'idée d'avoir un enfant, sans non plus rechercher activement une grossesse, pour lesquelles il est impossible de conclure quant à leur fertilité ou celle de leur couple s'il existe.

Afin de constituer un groupe infertile raisonnablement homogène, sa définition a finalement été restreinte aux cas où il y a eu "consultation pour rendre la grossesse possible" sans traitement de l'infertilité ultérieur (ce point est repris et développé dans la discussion). 
Figure 2 : Sélection de la population d'étude

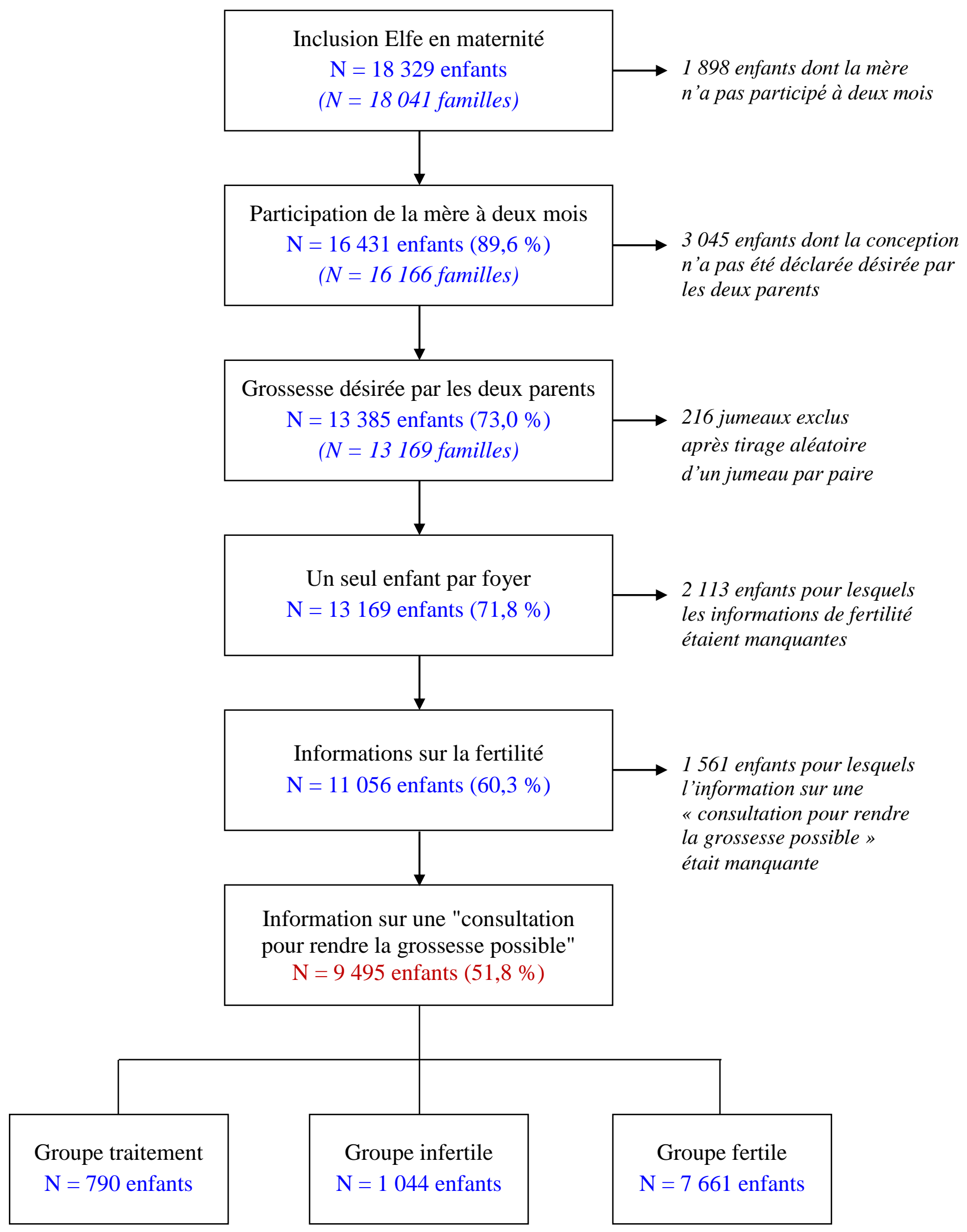




\subsection{PLAN D’ANALYSE}

\subsubsection{Caractéristiques de la population d'étude}

Une première étape de ce travail était de décrire et comparer l'état de santé des enfants et les circonstances de leur naissance dans les trois groupes d'étude. Différents indicateurs ont été analysés dans cinq champs :

- caractéristiques sociodémographiques des parents :

○ âge maternel, nationalité de la mère, niveau d'étude, catégorie socioprofessionnelle et statut de la mère vis-à-vis de l'emploi, couverture maladie de la mère, situation matrimoniale de la mère, ressources mensuelles du foyer ;

- antécédents maternels et contexte de la grossesse :

○ gestité et parité, antécédents d'hypertension ou de diabète, indice de masse corporelle, consommation alcoolo-tabagique, prise d'acide folique ;

- pathologies et complications pendant la grossesse :

O ressenti de la grossesse, hospitalisation pendant la grossesse, pathologie de la grossesse, pathologie fœtale ;

- travail et accouchement, état de santé des femmes et des enfants à la naissance :

○ gémellité, type de travail et d'accouchement, prématurité, petit poids de naissance, sexe, anomalie congénitale, réanimation en salle de naissance, score d'Apgar, hospitalisation ou transfert de la mère, transfert de l'enfant, traitement particulier en suites de couches, allaitement maternel ;

- état de santé des enfants à deux mois :

○ problème de santé et recours hospitalier dans les deux premiers mois, prise de poids, relation mère-enfant, allaitement maternel. 
Pour chaque item sont présentés les pourcentages pondérés et un test de comparaison global des proportions (test de $\chi^{2}$ de Pearson corrigé pour les données pondérées).

Une analyse en sous-groupes de traitement a été réalisée : une comparaison globale selon les trois types de traitement (FIV avec ou sans ICSI, insémination et stimulation), puis une comparaison des enfants issus de FIV (avec ou sans ICSI) et des enfants nés après stimulation, qui constitue un objectif secondaire de l'étude. Une comparaison FIV classique - ICSI a été faite avant de fusionner ces deux groupes distincts dans les données Elfe, ainsi qu'une comparaison deux à deux ICSI, FIV classique et stimulation (résultats présentés en annexe). Le groupe des inséminations artificielles n'a pas été étudié plus avant, ce d'autant qu'il n'a pas été possible à partir du questionnaire de distinguer les inséminations avec spermatozoïdes du conjoint de celles avec spermatozoïdes de donneur, qui représentent $11,6 \%$ des naissances après insémination [65]. La question ne se pose que dans une mesure bien moindre pour les naissances après FIV, issues d'un don de spermatozoïdes ou d'ovocytes dans 3,4 \% des cas seulement [65].

\subsubsection{Exploration de deux indicateurs de santé à la naissance}

Une deuxième étape était d'explorer les facteurs impactant sur l'état de santé des enfants. Deux indicateurs ont été considérés : la prématurité et le petit poids de naissance, codés en variables dépendantes binaires (âge gestationnel compris entre 33 et 37 semaines d'aménorrhée oui/non et poids de naissance inférieur à 2500 grammes oui/non).

Les indicateurs de santé à deux mois n'ont pas été explorés plus en détail en raison de l'absence de différence significative observée entre les groupes pour des variables subjectives peu discriminantes, et de la trop grande fragilité des informations collectées pour des variables plus objectives et pertinentes (formulation ambiguë des questions relatives au recours aux urgences, variable poids non renseignée dans près de la moitié des cas). 
La variable explicative principale considérée a été le contexte de la conception, considéré en trois classes (traitement / infertile sans traitement / fertile). Dans une analyse secondaire, la variable explicative principale était l'exposition à un traitement de l'infertilité, considéré en trois classes (pas d'infertilité / stimulation / FIV avec ou sans ICSI).

Les autres variables explicatives potentielles étaient les facteurs sociodémographiques (âge maternel, nationalité maternelle, niveau d'étude maternel, revenus mensuels du foyer, statut de couple de la mère), les facteurs médicaux (primiparité, IMC, diabète gestationnel, tabagisme maternel au dernier trimestre de la grossesse, sexe de l'enfant), la gémellité et la prématurité.

L'ensemble des variables explicatives potentielles ont été analysées en univarié puis retenues ou pas en multivarié au seuil de significativité de 0,10 pour être incluses dans un modèle de régression logistique. Les résultats sont exprimés en odds ratio. Pour chaque facteur, les odds ratios ont été comparés par le test de Wald adapté aux données pondérées.

L'ensemble des analyses ont été réalisées avec le logiciel STATA 13.

\section{Statut des variables explicatives potentielles dans la modélisation}

Beaucoup de facteurs sont potentiellement impliqués dans l'association entre le contexte de la conception et le sur-risque de prématurité ou de petit poids de naissance, mais leur rôle précis n'est pas toujours facile à modéliser. En effet la prise en compte de leurs effets doit se faire selon une stratégie d'analyse adaptée aux relations existant entre la variable explicative principale (le contexte de la conception et l'exposition à un traitement de l'infertilité), la variable à expliquer, et ces covariables d'ajustement.

Certaines sont des facteurs de confusion classiques (facteurs sociodémographiques, facteurs médicaux). La gémellité, en revanche, a un statut plus complexe. Ainsi, l'association entre le contexte de la conception et le sur-risque de prématurité pourrait être due en partie au 
sur-risque de grossesse gémellaire associé au traitement de l'infertilité, la gémellité pouvant être à la fois un facteur causal direct et un facteur intermédiaire dans la chaîne causale entre le traitement de l'infertilité et la prématurité (figure 3). La même difficulté se pose avec la prématurité dans l'étude du petit poids de naissance (figure 3). Les variables gémellité et prématurité soulèvent ainsi un problème particulier dans l'analyse. Cet effet de médiation est difficile à évaluer avec les méthodes de régression traditionnelles qui considèrent surtout l'effet de confusion.

Une approche possible est de stratifier les analyses selon le statut gémellaire (et prématuré) et de rechercher un effet d'interaction, mais cela ne permet pas de quantifier la contribution de la gémellité (et de la prématurité) au sur-risque de prématurité (et de petit poids de naissance) associé au traitement de l'infertilité.

Nous avons choisi ici de privilégier le rôle de facteur intermédiaire de la gémellité et de la prématurité, et de procéder par ajustements progressifs (facteurs sociodémographiques puis facteurs médicaux et enfin gémellité et prématurité). Nous avons de plus complété l'analyse par une étude des sous-populations singletons et singletons à terme. 
Figure 3 : Décomposition de l'effet total du traitement de l'infertilité sur le risque de prématurité et de petit poids de naissance en un effet direct (trait plein) et un effet indirect (trait pointillé) médié par la gémellité et la prématurité

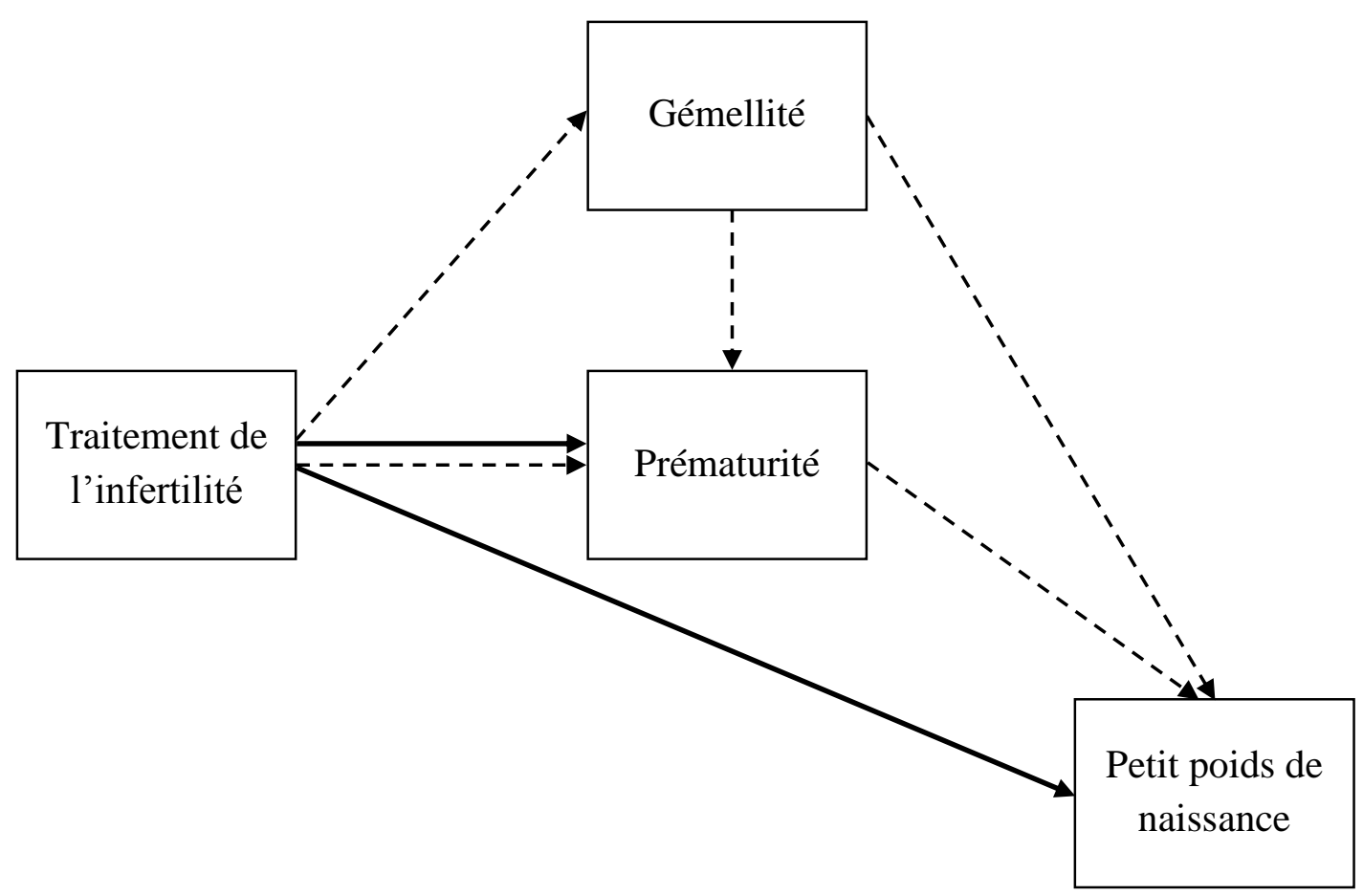




\subsubsection{Pondération}

Pour obtenir des résultats généralisables à l'ensemble de la population d'inférence de la cohorte Elfe (nourrissons nés en 2011 en maternité métropolitaine, singletons ou jumeaux, hors grands prématurés, de mère majeure parlant l'une des langues proposées et ne résidant pas temporairement en métropole), une pondération a été construite par l'équipe Elfe [93]. Elle consiste à assigner à chacun des nourrissons enquêtés un poids statistique qui correspond au nombre d'enfants qu'il représente dans la population visée, en prenant en compte deux sources de variabilité : le plan de sondage, et les phénomènes de non-réponse observés à différents niveaux.

Le plan de sondage de l'étude a été schématisé en trois phases d'échantillonnage : celle des maternités, celle des jours ou vagues de participation et celle des nourrissons :

- Les maternités ont été sélectionnées selon un plan de sondage à probabilités d'inclusion inégales stratifié sur la taille (allocation proportionnelle au nombre de naissances), afin de sur-représenter les grandes maternités : les maternités de petite taille ont été incluses à $25 \%$, les grandes maternités à $100 \%$. Un poids égal à 4 a donc été affecté aux premières et un poids égal à 1 aux secondes. Ces poids initiaux dus au plan de sondage ont ensuite été ajustés pour rendre compte de la non-participation de 29 maternités, grâce à quatre variables communes aux maternités répondantes et non-répondantes (région, statut juridique, strate de taille et niveau de médicalisation), selon la méthode des scores pondérés (un poids nul a été affecté aux maternités non-répondantes tandis que le poids des maternités répondantes a été réhaussé par un facteur d'ajustement).

- Les 25 jours d'enquête n'ont pas été tirés au sort mais choisis de manière à représenter plusieurs saisons et à coïncider en partie avec l'échantillon démographique permanent. La pondération utilisée dans le travail présenté ici ne corrigeait pas l'effet vague de naissance. Une nouvelle pondération prenant cet effet en compte a été publiée en juillet 
2015 : chaque nourrisson a été pondéré en fonction de sa vague de naissance, qui comportait un nombre de jours d'inclusion différent (4, 6, 7 et 8 jours). Les enfants nés lors de la première vague ont ainsi été inclus deux fois moins que ceux nés lors de la dernière vague, et ont donc reçu un poids deux fois plus grand. Les poids ont ensuite été ajustés pour rendre compte de la non-réponse (sur les $320 * 25=8000$ jours d'enquête attendus chez les maternités participantes, 7741 ont été effectués, soit 96,76\%).

- Les nourrissons de chaque maternité tirée ont été invités exhaustivement à participer à Elfe sous critères d'éligibilité. Cependant, seules $49 \%$ des mères ayant accouché les jours d'enquête ont accepté l'inclusion. La pondération a donc été ajustée pour rendre compte de la non-réponse des mères, en utilisant onze variables communes aux mères ayant refusé de participer et aux mères volontaires, et auxquelles la participation pouvait être liée (l'âge de la mère, son département, sa catégorie socioprofessionnelle, son activité au moment de la grossesse, la parité, la gémellité, l'âge gestationnel du nourrisson et les quatre variables caractérisant la maternité d'accouchement), selon la méthode des scores pondérés (un poids nul a été affecté aux nourrissons de mère nonrépondante tandis que le poids des nourrissons de mère répondante a été réhaussé par un facteur d'ajustement). Par exemple, les poids des nourrissons des mères ayant déjà eu un enfant ont été augmentés pour rendre compte de la moindre participation de ces mères par rapport aux mères primipares.

Au final, chaque nourrisson se voit donc affecté du poids corrigé de la maternité dans laquelle il est né, du poids temps associé à celle-ci ${ }^{1}$ et de son poids corrigé en fonction des caractéristiques des mères des nourrissons non participants.

\footnotetext{
${ }^{1}$ Dans la pondération publiée en juillet 2015 prenant cet effet en compte ; elle est décrite en figure 4.
} 
En second lieu, les poids ont été modifiés par un calage effectué sur les distributions de six variables provenant de l'état civil et de l'ENP 2010 (âge maternel, groupes de régions, primiparité, statut matrimonial, niveau d'études, statut immigré), afin d'assurer une meilleure représentativité géographique et sociodémographique de l'échantillon pondéré (malgré la pondération ajustée de la non-réponse, certaines catégories restaient sous-représentées, par exemple les mères très jeunes ou âgées), et de retrouver la structure de l'échantillon ENP considéré comme la référence.

La figure 4 résume la pondération de la cohorte Elfe au temps 0 (enquête en maternité). Au temps 1 (enquête à deux mois), la taille de l'échantillon des participants a diminué, et une nouvelle pondération a été construite [94], applicable à ce nouvel échantillon, c'est-à-dire aux nourrissons dont les familles ont participé aux deux temps d'enquête (maternité et deux mois).

\section{Figure 4 : Pondération de l'enquête Elfe en maternité}

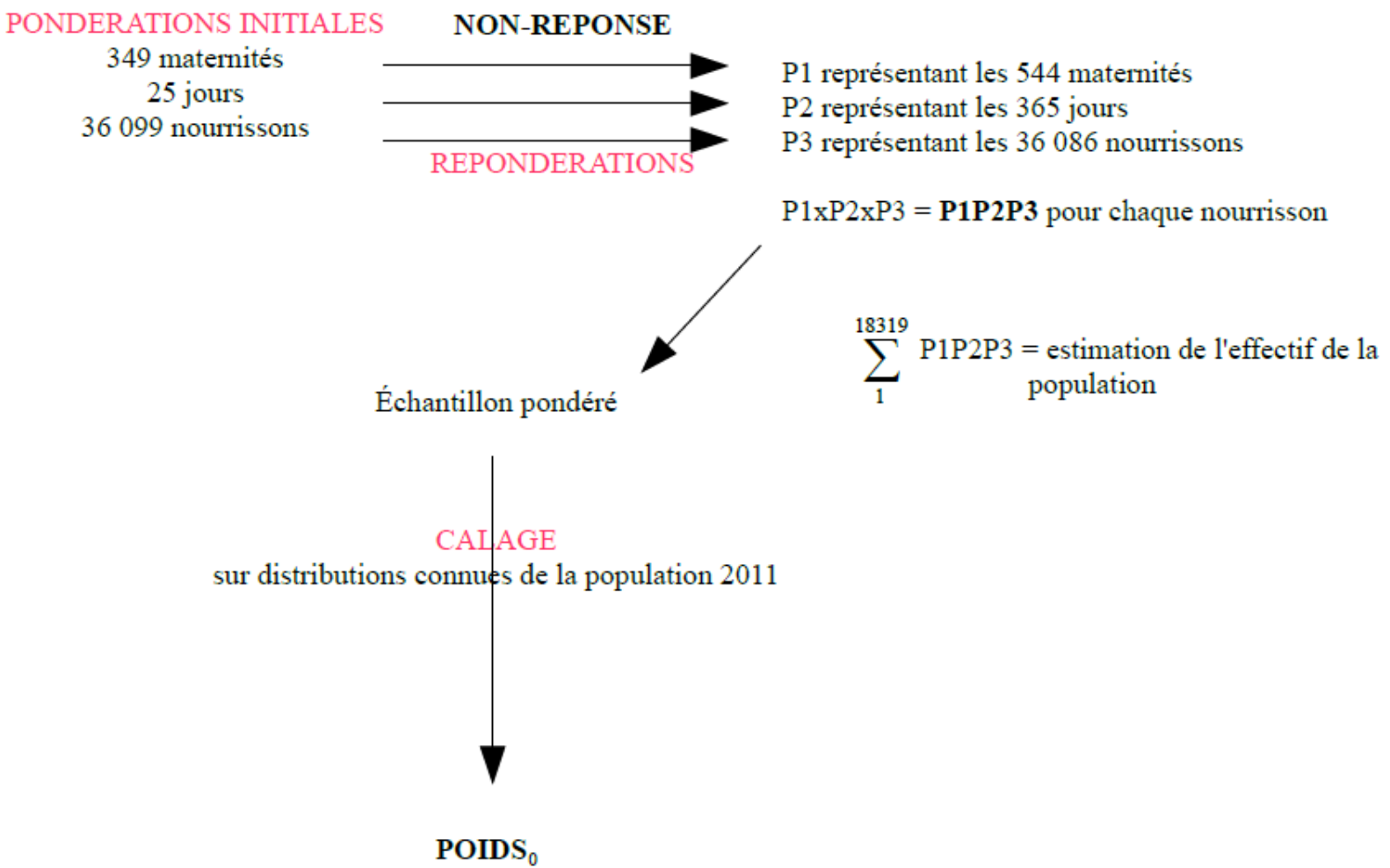

Source : pondération de l'enquête Elfe en maternité, note détaillée, Plateforme RE-CO-NAI, https://pandora.vjf.inserm.fr. 
L'utilisation de cette pondération dans Stata (option pweight) permet une estimation non biaisée des totaux d'intérêt. Mais elle ne suffit pas à estimer correctement la variance de ces totaux : pour cela il faut spécifier par une procédure appropriée (commande svy) le plan de sondage à l'origine des données (plan à plusieurs phases, stratifié, avec prise en compte de la non-réponse dans la pondération, et un calage), sous peine de sous-estimer les écarts-types.

Les variables nécessaires pour décrire le plan de sondage ne sont malheureusement pas encore accessibles dans la base de données fournie par l'équipe Elfe : elles ont en effet été classées «nominatives » ce qui rend une autorisation par la CNIL indispensable.

Les calculs ont par conséquent été effectués sur les données pondérées en utilisant dans un premier temps les procédures standards : les résultats présentés dans cette thèse sont donc préliminaires. Les calculs seront refaits dans un second temps en tenant compte du plan de sondage lorsque les données seront disponibles, pour une estimation précise de la variance. 


\section{RÉSULTATS}

\subsection{POPULATION D'ÉTUDE}

L'échantillon d'étude comporte 790 enfants dans le groupe traitement (232 ICSI, 115 FIV classiques, 144 inséminations et 299 stimulations), 1044 enfants dans le groupe infertile, et 7661 enfants dans le groupe fertile, soit un total de 9495 enfants analysés (figure 2) (effectifs bruts).

Ces effectifs sont conformes aux proportions observées lors de l'enquête nationale périnatale 2010 [2], comme le montre le tableau 2. Pour permettre la comparaison à l'ENP 2010, ce tableau porte sur l'ensemble de l'échantillon Elfe, avant restriction à un seul enfant en cas de naissance gémellaire, et les effectifs sont rapportés aux 16431 cas où la mère a complété le questionnaire à deux mois (qui comportait les informations relatives au traitement de l'infertilité). La part relative d'ICSI parmi les FIV est de $65 \%$, elle correspond aux chiffres de l'agence de la biomédecine pour l'année 2011 [65].

Tableau 2 : Quelques statistiques des naissances dans Elfe et l'ENP 2010

\begin{tabular}{|c|c|c|c|c|}
\hline & \multicolumn{2}{|c|}{ Elfe $(N=16431)^{1}$} & \multicolumn{2}{|c|}{ ENP $(\mathrm{N}=13677)$} \\
\hline & $\%$ & IC à $95 \%$ & $\%$ & IC à $95 \%$ \\
\hline conceptions issues d'une AMP & 3,4 & {$[3,1-3,7]$} & 3,3 & non précisé \\
\hline FIV classique & 0,8 & {$[0,7-1,0]$} & & \\
\hline FIV-ICSI & 1,4 & {$[1,2-1,6]$} & 2,3 & {$[2,0-2,5]$} \\
\hline$I A C / I A D$ & 1,1 & {$[1,0-1,3]$} & 1,0 & {$[0,7-1,1]$} \\
\hline conceptions issues d'une stimulation hormonale seule & 1,7 & {$[1,5-1,9]$} & 2,3 & {$[2,0-2,5]$} \\
\hline naissances gémellaires & 3,4 & {$[3,0-3,7]$} & 3,1 & {$[2,8-3,4]$} \\
\hline naissances prématurées 33-37 SA & 5,4 & {$[5,0-5,8]$} & 5,5 & non précisé \\
\hline petit poids de naissance & 5,5 & {$[5,0-5,9]$} & 5,3 & non précisé \\
\hline
\end{tabular}




\subsection{ANALYSE DESCRIPTIVE}

\subsubsection{Caractéristiques sociodémographiques parentales}

Les trois groupes d'étude diffèrent significativement pour l'ensemble des variables sociodémographiques analysées (tableau 3) : les mères du groupe traitement sont plus âgées, plus diplômées, plus actives et de catégorie socioprofessionnelle plus élevée que les mères des deux autres groupes. Elles bénéficient également d'une couverture maladie plus complète, sont plus souvent françaises de naissance et leur foyer présente des revenus mensuels plus importants. Elles sont plus souvent mariées/pacsées ou en couple.

Une comparaison des groupes d'étude deux à deux a été réalisée : un gradient sociodémographique significatif est observé dans le sens traitement-fertile-infertile (tableau A1 en annexe) pour la plupart des variables, notamment le niveau de diplôme et le niveau de revenus. Ces résultats montrent que le groupe témoin infertile diffère du groupe traitement autrement que par un niveau d'infertilité plus faible. 
Tableau 3 : Caractéristiques socio-économiques parentales selon le contexte de la conception

\begin{tabular}{ccc}
\hline groupe traitement & groupe infertile & groupe fertile \\
$(\mathrm{N}=790)$ & $(\mathrm{N}=1044)$ & $(\mathrm{N}=7661)$ \\
$\%$ & $\%$ & $\%$ \\
\hline
\end{tabular}

p global

âge maternel à la naissance

$\begin{array}{rccc}<25 \text { ans } & 2,4 & 9,6 & 12,5 \\ 25-35 \text { ans } & 65,5 & 61,5 & 72,5 \\ \geq 35 \text { ans } & 32,1 & 28,8 & 15,1\end{array}$

mère française de naissance

89,9

81,7

91,0

$<0,001$

diplôme le plus élevé obtenu par la mère

$\leq$ brevet des collèges

4,2

9,9

$<0,001$

$C A P$ / BEP

11,2

17,8

7,1

baccalauréat

13,8

18,6

13,7

$b a c+2$

27,3

22,1

17,6

$>b a c+2$

43,5

31,6

23,1

38,6

CSP maternelle élevée ${ }^{1}$

56,6

45,8

49,4

$<0,001$

emploi lors de la grossesse

88,9

74,4

80,0

$<0,001$

couverture maladie maternelle

$C M U$ / AME / aucun régime

2,5

9,2

$<0,001$

régime sans complémentaire

4,8

6,6

4,9

régime et complémentaire

92,7

84,2

5,5

89,6

revenus mensuels du foyer

$$
\begin{array}{r}
<2500 \text { euros } \\
2500-3999 \text { euros } \\
\geq 4000 \text { euros }
\end{array}
$$$$
16,5
$$$$
46,3
$$

37,2

23,3

49,4

25,2

état matrimonial : mère mariée ou pacsée

80,5

63,6

62,4

$<0,001$

vie en couple à la naissance

99,9

98,4

98,9

0,022

${ }^{1}$ CSP élevée : agriculteur/exploitant, artisan/commerçant/chef d'entreprise, cadre/profession intellectuelle supérieure, profession intermédiaire (catégories Insee). 


\subsubsection{Antécédents maternels et contexte de la grossesse}

La gestité et la parité diffèrent significativement entre les trois groupes : les mères du groupe traitement sont plus souvent primigestes et primipares (tableau 4).

Les mères du groupe traitement ont un comportement moins à risque et plus préventif, avec une consommation alcoolo-tabagique moindre pendant la grossesse et une supplémentation en acide folique plus fréquente.

Les trois groupes ne différent pas quant à leurs antécédents d'hypertension et de diabète.

En revanche, le groupe infertile présente une proportion plus élevée d'IMC extrême.

Tableau 4 : Antécédents maternels et contexte de la grossesse selon le contexte de la conception

\begin{tabular}{|c|c|c|c|c|}
\hline & $\begin{array}{l}\text { groupe traitement } \\
\qquad(\mathrm{N}=790)\end{array}$ & $\begin{array}{l}\text { groupe infertile } \\
\qquad(\mathrm{N}=1044)\end{array}$ & $\begin{array}{l}\text { groupe fertile } \\
(\mathrm{N}=7 \text { 661) }\end{array}$ & \\
\hline & $\%$ & $\%$ & $\%$ & $\mathrm{p}$ global \\
\hline grossesse antérieure & 60,6 & 67,0 & 64,5 & 0,045 \\
\hline nombre d'enfants antérieurs & & & & $<0,001$ \\
\hline 0 & 63,9 & 57,5 & 47,9 & \\
\hline 1 & 28,8 & 28,8 & 36,7 & \\
\hline$\geq 2$ & 7,3 & 13,7 & 15,4 & \\
\hline HTA (chronique ou gestationnelle) & 1,3 & 2,3 & 2,2 & 0,398 \\
\hline diabète (chronique ou gestationnel) & 3,0 & 3,9 & 3,1 & 0,522 \\
\hline IMC maternel avant grossesse $\left(\mathrm{kg} / \mathrm{m}^{2}\right)$ & & & & 0,007 \\
\hline$<18,5$ & 8,6 & 9,6 & 7,1 & \\
\hline $18,5-24,9$ & 69,2 & 61,7 & 66,4 & \\
\hline $25-29,9$ & 15,1 & 17,0 & 17,4 & \\
\hline$\geq 30$ & 7,1 & 11,7 & 9,0 & \\
\hline tabagisme pendant la grossesse & 11,2 & 18,6 & 18,2 & $<0,001$ \\
\hline tabagisme pendant le $3^{\text {ème }}$ trimestre & 8,8 & 15,0 & 14,2 & 0,003 \\
\hline consommation d'alcool lors de la grossesse & 12,6 & 14,9 & 18,8 & $<0,001$ \\
\hline acide folique avant/pendant la grossesse & 70,7 & 48,4 & 43,5 & $<0,001$ \\
\hline
\end{tabular}


Étonnamment, le groupe infertile tend à avoir plus de mères non primigestes que le groupe fertile. Néanmoins, cette tendance n'est pas significative dans la comparaison des groupes deux à deux ( $\mathrm{p}=0,170$, tableau $\mathrm{A} 2)$. Par ailleurs, la comparaison du nombre d'enfants ne montre pas de tendance similaire, ce qui suggère l'hypothèse de grossesses interrompues plus fréquentes dans le groupe infertile. Cette hypothèse est soutenue par le fait que 38,9 \% des mères du groupe infertile ont fait une fausse couche pendant la période où elles ont essayé de concevoir l'enfant inclus dans l'étude, contre $10,9 \%$ dans le groupe fertile $(\mathrm{p}<0,001$, tableau A2). 


\subsubsection{Pathologies et complications pendant la grossesse}

Les mères du groupe traitement sont plus nombreuses à avoir été heureuses en apprenant leur grossesse (tableau 5), mais elles tendent à éprouver plus de difficultés psychologiques que les mères du groupe fertile ( $\mathrm{p}=0,062$, tableau $\mathrm{A} 3$ ).

La grossesse apparaît également plus compliquée : la proportion de mères hospitalisées est presque de 10 points supérieure à celle du groupe fertile. Les pathologies de la grossesse sont en effet plus fréquentes, avec davantage de diabète gestationnel, de menace d'accouchement prématuré grave entraînant une corticothérapie anténatale, et de placenta prævia hémorragique (complication d'une anomalie d'insertion du placenta). Il ne semble pas en revanche y avoir de répercussion sur le fœtus.

Tableau 5 : Pathologies et complications de la grossesse selon le contexte de la conception

\begin{tabular}{|c|c|c|c|c|}
\hline & $\begin{array}{c}\text { groupe traitement } \\
\qquad(\mathrm{N}=790) \\
\%\end{array}$ & $\begin{array}{c}\text { groupe infertile } \\
(\mathrm{N}=1044) \\
\%\end{array}$ & $\begin{array}{c}\text { groupe fertile } \\
(\mathrm{N}=7 \text { 661) } \\
\%\end{array}$ & p global \\
\hline mère heureuse d'être enceinte & 99,2 & 96,0 & 94,8 & $<0,001$ \\
\hline $\begin{array}{l}\text { difficultés psychologiques durant la } \\
\text { grossesse }\end{array}$ & 12,2 & 14,0 & 9,9 & $<0,001$ \\
\hline hospitalisation pendant la grossesse & 24,2 & 20,9 & 14,7 & $<0,001$ \\
\hline pathologie de la grossesse $^{1}$ & 32,0 & 27,4 & 23,9 & $<0,001$ \\
\hline diabète gestationnel & 9,3 & 8,1 & 6,3 & 0,010 \\
\hline placenta pravia hémorragique & 1,3 & 0,5 & 0,3 & $<0,001$ \\
\hline menace d'accouchement prématuré & 11,1 & 9,7 & 7,3 & 0,002 \\
\hline corticothérapie anténatale & 8,9 & 6,0 & 3,6 & $<0,001$ \\
\hline pathologie fotale $^{2}$ & 4,2 & 3,1 & 3,2 & 0,448 \\
\hline
\end{tabular}

${ }^{1}$ Pathologie de la grossesse : menace d'accouchement prématuré, rupture prématurée des membranes, hémorragie du $2^{\text {ème }}$ ou du $3^{\text {ème }}$ trimestre, HTA gestationnelle, diabète gestationnel.

${ }^{2}$ Pathologie fotale : RCIU, anomalie congénitale, infection congénitale (toxoplasmose, CMV). 
Une comparaison des groupes deux à deux montre que les mères du groupe infertile éprouvent plus de difficultés psychologiques que les mères du groupe fertile $(p<0,001)$, sont significativement plus souvent hospitalisées $(\mathrm{p}<0,001)$ et présentent davantage de menace d'accouchement prématuré ( $\mathrm{p}=0,030)$ (tableau A3).

\subsubsection{Travail et accouchement, état de santé des femmes et des enfants à la naissance.}

De façon tout à fait conforme à la littérature, le taux de gémellité est très supérieur dans le groupe traitement, et s'accompagne d'une plus forte proportion de déclenchement du travail et de césarienne (tableau 6). Les taux d'enfants prématurés et d'enfants de petit poids sont également plus élevés. La proportion de garçons et le taux d'anomalie congénitale diagnostiquée en salle de naissance sont les mêmes dans les trois groupes.

Il n'y a pas de différence globale significative quant à la pratique de geste de réanimation en salle de naissance, mais une comparaison des groupes traitement et fertile montre un surrisque du groupe traitement significatif $(\mathrm{p}=0,013$, tableau $\mathrm{A} 4)$. Le groupe infertile présente également significativement plus de césariennes $(\mathrm{p}<0,001)$ et de prématurés $(\mathrm{p}=0,007)$ que le groupe fertile (tableau A4).

En suites de couches immédiates, le taux de transfert de l'enfant diffère significativement entre les trois groupes, il est plus élevé dans les groupes traitement et infertile. Ce n'est pas le cas du taux d'hospitalisation maternelle. Le gavage est plus fréquent dans le groupe traitement, résultat qui peut être relié aux enfants de petits poids de naissance plus nombreux. La pratique de l'allaitement est similaire dans les trois groupes.

Un chiffre surprenant est la proportion plus élevée d'enfants de score de vitalité d'Apgar anormal à 5 minutes dans le groupe infertile, avec une différence globale significative due complètement au groupe infertile. Il en va de même pour l'administration d'antibiothérapie intraveineuse plus fréquente dans le groupe infertile (tableau A4). 
Tableau 6 : Travail et accouchement, état de santé des mères et des enfants à la naissance et en suites de couches immédiates selon le contexte de la conception

\begin{tabular}{|c|c|c|c|c|}
\hline & \multirow{3}{*}{$\begin{array}{c}\text { groupe traitement } \\
(\mathrm{N}=790) \\
\% \\
\end{array}$} & \multirow{3}{*}{$\begin{array}{c}\text { groupe infertile } \\
(\mathrm{N}=1044) \\
\% \\
\end{array}$} & \multirow{3}{*}{$\begin{array}{c}\text { groupe fertile } \\
(\mathrm{N}=7661) \\
\% \\
\end{array}$} & \multirow[b]{3}{*}{$\mathrm{p}$ global } \\
\hline & & & & \\
\hline & & & & \\
\hline gémellité & 12,3 & 1,0 & 1,2 & $<0,001$ \\
\hline travail spontané & 64,4 & 69,8 & 73,4 & $<0,001$ \\
\hline type d'accouchement & & & & $<0,001$ \\
\hline voie basse spontanée & 58,1 & 59,5 & 70,3 & \\
\hline voie basse instrumentale & 14,0 & 16,4 & 13,2 & \\
\hline césarienne & 27,9 & 24,1 & 16,5 & \\
\hline prématurité & 10,4 & 6,4 & 4,1 & $<0,001$ \\
\hline petit poids de naissance & 9,2 & 5,2 & 4,0 & $<0,001$ \\
\hline sexe masculin & 52,4 & 51,4 & 50,7 & 0,723 \\
\hline anomalie congénitale & 2,4 & 2,1 & 2,6 & 0,711 \\
\hline réanimation en salle de naissance & 4,6 & 3,9 & 2,9 & 0,064 \\
\hline score d'Apgar à 5 minutes $\leq 7$ & 1,5 & 3,0 & 1,2 & $<0,001$ \\
\hline hospitalisation / transfert de la mère & 1,3 & 1,8 & 1,0 & 0,151 \\
\hline transfert de l'enfant & 8,0 & 7,4 & 4,9 & $<0,001$ \\
\hline traitement particulier en suites de couches ${ }^{1}$ & 8,4 & 9,0 & 6,7 & 0,027 \\
\hline antibiothérapie intraveineuse & 1,6 & 3,7 & 1,7 & $<0,001$ \\
\hline gavage & 3,2 & 2,1 & 1,4 & 0,020 \\
\hline allaitement maternel (complet ou mixte) & 71,4 & 70,2 & 67,7 & 0,104 \\
\hline
\end{tabular}

\footnotetext{
${ }^{1}$ Traitement particulier en suites de couches : photothérapie, antibiothérapie intraveineuse, gavage.
} 


\subsection{5. État de santé des enfants à deux mois}

La quasi-totalité des enfants sont en bonne santé à deux mois d'après leur mère quel que soit leur groupe (tableau 7). Près des trois quarts ont présenté un problème de santé au sens très large, allant d'un simple rhume à une pneumonie par exemple. Cette proportion est significativement plus élevée dans les groupes traitement et infertile.

La prise de poids, indicateur de santé et de développement plus objectif, n'a malheureusement pu être reconstituée que dans moins de la moitié des cas ; elle ne diffère pas selon les groupes. Le recours hospitalier (au moins une consultation aux urgences ou une hospitalisation au cours des deux premiers mois) ne diffère pas non plus significativement, ni le critère composite de bonne relation mère-enfant ou le taux d'allaitement maternel, qui a dans les trois groupes diminué de plus de 20 points par rapport au taux observé en maternité.

Les comparaisons des groupes deux à deux retrouvent une différence globale significative du recours aux urgences due complètement au groupe infertile (tableau A5). 
Tableau 7 : État de santé des enfants à deux mois selon le contexte de la conception

\begin{tabular}{|c|c|c|c|}
\hline $\begin{array}{c}\text { groupe traitement } \\
\qquad(\mathrm{N}=790) \\
\%\end{array}$ & $\begin{array}{c}\text { groupe infertile } \\
(\mathrm{N}=1044) \\
\%\end{array}$ & $\begin{array}{c}\text { groupe fertile } \\
(\mathrm{N}=7661) \\
\%\end{array}$ & p global \\
\hline 99,3 & 98,4 & 99,2 & 0,125 \\
\hline 74,5 & 75,4 & 71,1 & 0,018 \\
\hline & & & 0,123 \\
\hline 20,6 & 23,4 & 24,6 & \\
\hline 72,3 & 65,7 & 67,5 & \\
\hline 7,1 & 10,9 & 7,9 & \\
\hline 16,1 & 17,3 & 14,9 & 0,185 \\
\hline 12,0 & 15,0 & 11,8 & 0,036 \\
\hline 7,2 & 4,6 & 5,8 & 0,084 \\
\hline 76,0 & 74,4 & 77,7 & 0,102 \\
\hline 93,2 & 94,4 & 92,8 & 0,190 \\
\hline 99,4 & 98,4 & 99,2 & 0,106 \\
\hline 96,1 & 95,0 & 95,3 & 0,645 \\
\hline 94,4 & 94,2 & 95,8 & 0,050 \\
\hline 87,3 & 86,0 & 90,2 & $<0,001$ \\
\hline 46,4 & 47,3 & 45,2 & 0,492 \\
\hline
\end{tabular}

${ }^{1}$ D'après la question posée à la mère "selon vous, votre enfant est-il actuellement : en bonne santé ou plutôt en bonne santé / plutôt en mauvaise santé ou en mauvaise santé".

${ }^{2} \mathrm{Au}$ moins une préoccupation ou un problème de santé dans les deux premiers mois déclaré par la mère parmi : problème d'alimentation ou de poids, ictère, régurgitation ou reflux gastro-œsophagien, coliques ou diarrhée ou gastro-entérite ou constipation, malaise, fièvre, rhinopharyngite, conjonctivite, muguet, infection, choc ou traumatisme, symptôme respiratoire, éruption cutanée, allergie aux protéines de lait de vache, autre.

${ }^{3}$ La mère a répondu oui aux cinq items (chant, parole, regard, sourire, cause des pleurs). 


\subsubsection{Description par sous-groupes de traitement de l'infertilité}

L'analyse porte sur les 790 enfants du groupe traitement, composé des enfants conçus par FIV avec ou sans ICSI $(n=347)$, des enfants conçus par IAC et IAD $(n=144)$ et des enfants conçus à la suite d'une simple stimulation hormonale (n=299) (effectifs bruts).

Les foyers présentent des caractéristiques sociodémographiques similaires dans les trois groupes, hormis pour l'âge maternel, globalement plus élevé dans les groupes AMP (FIV avec ou sans ICSI et insémination) qu'en stimulation (tableau A6).

Les mères diffèrent peu globalement quant à leurs antécédents. Une proportion plus importante de femmes dont il s'agit du premier enfant dans les groupes AMP, et un antécédent de diabète plus fréquent dans le groupe stimulation, sont à noter cependant. L'antécédent de tabagisme avant la grossesse tend à être plus élevé dans les groupes AMP, ce n'est pas le cas pendant la grossesse (tableau A7).

Dans le champ des pathologies et complications pendant la grossesse, les mères des groupes AMP sont globalement plus souvent hospitalisées qu'en stimulation. On observe notamment davantage de placenta prævia hémorragique en cas de FIV \pm ICSI (tableau A8).

Les résultats portant sur l'accouchement et l'état de santé des femmes et des enfants à la naissance sont bien plus contrastés (tableau 8). Le taux de gémellité est globalement très supérieur dans les groupes AMP, s'accompagnant d'une plus forte proportion de déclenchement et de césarienne dans le groupe FIV \pm ICSI. Les taux de prématurité et de petit poids de naissance tendent à différer globalement, et il y a significativement plus d'enfants prématurés après FIV \pm ICSI en comparaison avec le groupe stimulation $(13,6 \%$ contre $6,4 \%, p=0,006$, tableau A9). Davantage de gestes de réanimation à la naissance ont été pratiqués sur les enfants du groupe FIV \pm ICSI, la différence est significative également dans la comparaison avec le groupe stimulation ( $\mathrm{p}=0,021$, tableau $\mathrm{A} 9)$. Les mères sont plus souvent hospitalisées dans le 
groupe FIV \pm ICSI que dans les deux autres groupes, avec une différence globale significative qui est retrouvée dans la comparaison avec le groupe stimulation (tableau A9).

Tableau 8 : Travail et accouchement, état de santé des mères et des enfants à la naissance et en suites de couches selon le groupe de traitement de l'infertilité

\begin{tabular}{|c|c|c|c|c|}
\hline & $\begin{array}{c}\text { FIV } \pm \text { ICSI } \\
(\mathrm{N}=347) \\
\%\end{array}$ & $\begin{array}{c}\text { IAC/IAD } \\
(\mathrm{N}=144) \\
\% \\
\end{array}$ & $\begin{array}{c}\text { stimulation } \\
(\mathrm{N}=299) \\
\%\end{array}$ & $\underset{\text { global }}{\mathrm{p}}$ \\
\hline gémellité & 16,1 & 17,6 & 4,9 & $<0,001$ \\
\hline travail spontané & 55,8 & 72,9 & 70,0 & $<0,001$ \\
\hline type d'accouchement & & & & $<0,001$ \\
\hline voie basse spontanée & 49,4 & 61,8 & 66,6 & \\
\hline voie basse instrumentale & 14,1 & 17,0 & 12,2 & \\
\hline césarienne & 36,5 & 21,2 & 21,2 & \\
\hline prématurité & 13,6 & 10,7 & 6,4 & 0,060 \\
\hline petit poids de naissance & 10,4 & 13,7 & 5,2 & 0,057 \\
\hline sexe masculin & 54,4 & 45,3 & 54,0 & 0,249 \\
\hline anomalie congénitale & 1,4 & 6,6 & 1,4 & 0,007 \\
\hline réanimation en salle de naissance & 6,9 & 2,9 & 2,8 & 0,030 \\
\hline score d'Apgar à 5 minutes $\leq 7$ & 1,4 & 0 & 2,3 & 0,189 \\
\hline hospitalisation / transfert de la mère & 2,4 & 0,5 & 0,3 & 0,015 \\
\hline transfert de l'enfant & 9,5 & 7,6 & 6,3 & 0,438 \\
\hline traitement particulier en suites de couches ${ }^{1}$ & 10,4 & 6,2 & 7,3 & 0,292 \\
\hline photothérapie & 6,8 & 3,7 & 5,8 & 0,486 \\
\hline gavage & 4,4 & 3,9 & 1,1 & 0,118 \\
\hline allaitement maternel (complet ou mixte) & 72,7 & 65,9 & 72,9 & 0,349 \\
\hline
\end{tabular}

${ }^{1}$ Traitement particulier en suites de couches : photothérapie, antibiothérapie intraveineuse, gavage.

Aucune différence significative n'est constatée sur l'état de santé des enfants à deux mois (tableau A10). 


\subsection{ANALYSE PAR INDICATEURS}

\subsubsection{Prématurité}

L'analyse univariée a permis dans un premier temps de décrire le contexte de la conception (enfant conçu grâce à un traitement de l'infertilité, suite à une période d'infécondité sans qu'un traitement ait été nécessaire, ou sans qu'il y ait eu de période d'infécondité), dont on cherche à comprendre l'effet, et les facteurs de risque de la prématurité décrits dans la littérature (facteurs sociaux et comportementaux : nationalité, faible niveau d'études de la mère, faible niveau de ressources du foyer, statut monoparental, consommation de tabac durant le troisième trimestre de la grossesse ; âge maternel extrême, c'est-à-dire inférieur à 25 ans ou supérieur à 35 ans ; grossesse multiple ; autres facteurs médicaux : faible poids de la mère avant la grossesse, nulliparité ou grande multiparité, grossesse pathologique) [72]. Toutes les variables ayant un degré de signification inférieur à 0,10 ont ensuite été incluses dans un modèle multivarié par régression logistique, ainsi que l'âge maternel.

Après ajustement, les liens connus entre prématurité et niveau de diplôme maternel, statut monoparental, et diabète gestationnel sont retrouvés ; la gémellité apparaît comme le facteur le plus fortement associé à la prématurité, avec un OR de 13,7 très significatif (tableau 9). L'association décrite dans la littérature avec l'âge maternel n'apparaît ni en univarié ni en multivarié, de même que l'association avec la nationalité. Le lien avec le tabagisme au troisième trimestre de la grossesse est à la limite de la significativité. Le lien avec l'indice de masse corporelle maternel disparaît après ajustement. 
Tableau 9 : Analyse des facteurs associés à la prématurité (modèle logistique complet)

\begin{tabular}{|c|c|c|c|c|c|}
\hline \multicolumn{3}{|c|}{ analyse univariée ${ }^{1}$} & \multicolumn{3}{|c|}{$\begin{array}{l}\text { analyse multivariée } \\
\qquad(\mathrm{n}=8 \text { 503) }\end{array}$} \\
\hline OR & IC à $95 \%$ & $\mathrm{p}$ & OR & IC à $95 \%$ & $\mathrm{p}$ \\
\hline & & $<0,001$ & & & 0,008 \\
\hline 2,70 & {$[1,97-3,70]$} & $<0,001$ & 1,53 & {$[1,07-2,19]$} & 0,020 \\
\hline 1,58 & {$[1,13-2,19]$} & 0,007 & 1,52 & {$[1,08-2,14]$} & 0,017 \\
\hline réf & - & - & réf & - & - \\
\hline
\end{tabular}

âge maternel à la naissance

$$
\begin{array}{r}
<25 \text { ans } \\
25-35 \text { ans } \\
\geq 35 \text { ans }
\end{array}
$$$$
0,226
$$

0,226

$\begin{array}{rr}\mathbf{0 , 6 0 1} \\ \mathbf{1 , 1 0}[0,72-1,67] & 0,670\end{array}$

1,20

$0,83-1,72]$

0,331

réf

$\mathbf{1 , 2 6}[0,95-1,68] \quad 0,115$

réf

$\mathbf{1 , 1 7}[0,85-1,60] \quad 0,335$

mère non française de naissance

$\mathbf{0 , 7 8}[0,51-1,20] \quad \mathbf{0 , 2 5 9}$

diplôme le plus élevé de la mère

$\begin{array}{ccc} & & <\mathbf{0 , 0 0 1} \\ \mathbf{1 , 8 9} & {[1,37-2,61]} & <0,001 \\ \mathbf{1 , 5 3} & {[1,20-1,94]} & 0,001 \\ \text { réf } & - & -\end{array}$

\section{1,5}

$\mathbf{0 , 0 1 1}$

$\leq$ brevet / CAP / BEP

$>b a c+2$

revenus mensuels du foyer

$$
\begin{aligned}
& <2500 \text { euros } \\
2500 & -3999 \text { euros } \\
& >4000 \text { euros }
\end{aligned}
$$

$\begin{array}{ccc}\mathbf{1 , 1 8} & {[0,89-1,57]} & \mathbf{0 , 0 6 6} \\ \text { réf } & - & - \\ \mathbf{0 , 8 0} & {[0,61-1,05]} & 0,114\end{array}$

statut monoparental de la mère à la naissance

$\mathbf{3 , 1 3}[1,29-7,59] \quad \mathbf{0 , 0 1 2}$

gémellité

15,8

$\mathbf{1 , 2 0}[0,95-1,51] \quad \mathbf{0 , 1 2 8}$

primiparité

IMC maternel avant grossesse $\left(\mathrm{kg} / \mathrm{m}^{2}\right)$

$$
\begin{array}{r}
<18,5 \\
18,5-24,9 \\
25-29,9 \\
\geq 30
\end{array}
$$

1,35

$\mathbf{0 , 0 3 5}$

1,41

réf

0,010

$41,09-1,83] \quad 0,010$

- 
Le contexte de la conception est significativement associé à la prématurité en univarié et le reste après ajustement sur les autres variables : le tableau 10 rapporte l'évolution des OR associés au contexte de la conception après ajustement progressif sur les caractéristiques sociodémographiques, les facteurs médicaux, et la gémellité (facteur le plus fortement associé à la prématurité). L'entrée de la gémellité dans le modèle ne fait pas disparaître l'association globale, mais elle annule la différence entre les groupes traitement et infertile (OR de 1,80 significatif dans le modèle 2 , égal à 1 dans le modèle suivant). Ce résultat est confirmé par l'analyse restreinte aux singletons (tableau 10) : il existe une association significative entre contexte de la conception et prématurité, sans différence significative entre les groupes traitement et infertile.

Tableau 10 : Odds Ratio de prématurité lié au contexte de la conception selon le modèle logistique et la population d'analyse

\begin{tabular}{|c|c|c|c|c|c|c|c|}
\hline & \multirow{2}{*}{\multicolumn{3}{|c|}{ population totale }} & & & \\
\hline & & & & & \multicolumn{3}{|c|}{ singletons } \\
\hline & & $\mathrm{OR}$ & IC à $95 \%$ & $\mathrm{p}$ & $\mathrm{OR}$ & IC à $95 \%$ & $\mathrm{p}$ \\
\hline \multirow[t]{4}{*}{ analyse univariée } & & & \multicolumn{2}{|c|}{$\mathbf{p}<0,001$} & \multicolumn{3}{|c|}{$\mathbf{p}=\mathbf{0 , 0 0 1}$} \\
\hline & traitement vs fertile & 2,70 & {$[1,97-3,70]$} & $<0,001$ & 1,64 & {$[1,17-2,31]$} & 0,004 \\
\hline & infertile vs fertile & 1,58 & {$[1,13-2,19]$} & 0,007 & 1,65 & {$[1,16-2,33]$} & 0,005 \\
\hline & traitement vs infertile & 1,71 & {$[1,13-2,59]$} & 0,011 & 1,00 & {$[0,64-1,55]$} & 0,991 \\
\hline
\end{tabular}

modèle multivarié

1 : facteurs sociodémographiques traitement vs fertile infertile vs fertile traitement vs infertile

$2: \ldots+$ facteurs médicaux traitement vs fertile infertile vs fertile traitement vs infertile

$3:$... + gémellité

traitement vs fertile infertile vs fertile traitement vs infertile

$$
\mathbf{p}<\mathbf{0 , 0 0 1}
$$

$2,68 \quad[1,96-3,67]<0,001$

$1,46 \quad[1,04-2,05] \quad 0,028$

$1,83 \quad[1,21-2,78] \quad 0,004$

$$
\mathbf{p}<\mathbf{0 , 0 0 1}
$$

$2,71 \quad[1,97-3,73]<0,001$

$1,50 \quad[1,08-2,10] \quad 0,017$

$1,80 \quad[1,19-2,73] \quad 0,005$

$$
\mathbf{p}=\mathbf{0 , 0 0 8}
$$

$1,53 \quad[1,07-2,19] \quad 0,020$

$1,52 \quad[1,08-2,14] \quad 0,017$

$1,01 \quad[0,64-1,59] \quad 0,977$ 


\subsubsection{Petit poids de naissance}

L'analyse a été conduite selon le même principe pour explorer les facteurs associés au petit poids de naissance.

Après ajustement, les liens connus entre petit poids de naissance et niveau de diplôme maternel, primiparité, IMC maternel avant la grossesse et sexe féminin [74] sont retrouvés ; la gémellité et la prématurité apparaissent comme les facteurs les plus fortement associés au petit poids de naissance, avec des OR très significatifs de 17,5 et 24,2 respectivement (tableau 11). Les associations avec l'âge maternel et le tabagisme au troisième trimestre de la grossesse sont à la limite de la significativité.

Il n'y a pas de lien entre le contexte de la conception et le petit poids de naissance après prise en compte des facteurs sociaux et médicaux, de la gémellité et de la prématurité. 
Tableau 11 : Analyse des facteurs associés à un petit poids de naissance (modèle logistique complet)

\begin{tabular}{|c|c|c|c|c|c|}
\hline \multicolumn{3}{|c|}{ analyse univariée $^{1}$} & \multicolumn{3}{|c|}{$\begin{array}{c}\text { analyse multivariée } \\
(\mathrm{n}=7687)\end{array}$} \\
\hline OR & IC à $95 \%$ & $\mathrm{p}$ & OR & IC à $95 \%$ & $\mathrm{p}$ \\
\hline
\end{tabular}

contexte de la conception

traitement
infertile
fertile

âge maternel à la naissance

$$
\begin{array}{r}
<25 \text { ans } \\
25-35 \text { ans } \\
\geq 35 \text { ans }
\end{array}
$$

mère non française de naissance

diplôme le plus élevé de la mère

$$
\begin{array}{r}
\leq \text { brevet / CAP / BEP } \\
\text { baccalauréat à } b a c+2 \\
>b a c+2
\end{array}
$$

revenus mensuels du foyer

$$
\begin{array}{r}
<2500 \text { euros } \\
2500-3999 \text { euros } \\
>4000 \text { euros }
\end{array}
$$

statut monoparental de la mère à la naissance

gémellité

prématurité

primiparité

IMC maternel avant grossesse $\left(\mathrm{kg} / \mathrm{m}^{2}\right)$

$$
\begin{array}{r}
<18,5 \\
18,5-24,9 \\
25-29,9 \\
\geq 30
\end{array}
$$

tabagisme au $3^{\text {ème }}$ trimestre de la grossesse

sexe féminin

$$
<\mathbf{0 , 0 0 1}
$$

$\mathbf{2 , 4 0}[1,69-3,41]<0,001$

$\mathbf{1 , 3 1}[0,92-1,88] \quad 0,139$

réf

$\begin{array}{ccc} & & \mathbf{0 , 3 7 7} \\ \mathbf{0 , 7 1} & {[0,39-1,29]} & 0,258 \\ \mathbf{0 , 7 9} & {[0,49-1,27]} & 0,332 \\ \text { réf } & - & -\end{array}$

$\begin{array}{ccc}\mathbf{1 , 0 9} & {[0,73-1,63]} & \mathbf{0 , 8 1 1} \\ \text { réf } & - & - \\ \mathbf{1 , 0 9} & {[0,81-1,47]} & 0,574 \\ & & \\ \mathbf{0 , 8 5} & {[0,53-1,36]} & \mathbf{0 , 4 9 7} \\ & & \\ & & <\mathbf{0 , 0 0 1} \\ \mathbf{2 , 2 0} & {[1,61-3,02]} & <0,001 \\ \mathbf{1 , 2 9} & {[0,99-1,69]} & 0,059 \\ \text { réf } & - & -\end{array}$

$\begin{array}{ccc} & & \mathbf{0 , 0 6 4} \\ \mathbf{0 , 6 1} & {[0,37-1,00]} & 0,050 \\ \text { réf } & - & - \\ \mathbf{1 , 2 4} & {[0,81-1,90]} & 0,319\end{array}$

$1,24-[0,81-1,90] \quad 0,319$

$\begin{array}{ccc}\mathbf{1 , 4 2} & {[1,05-1,91]} & \mathbf{0 , 0 2 4} \\ \text { réf } & - & -022 \\ \mathbf{0 , 9 2} & {[0,69-1,23]} & 0,583\end{array}$

$\begin{array}{ccc}\mathbf{2 , 2 1} & {[1,41-3,48]} & \mathbf{0 , 0 0 2} \\ \mathbf{1 , 1 7} & {[0,84-1,64]} & 0,347 \\ \text { réf } & - & -\end{array}$

$\mathbf{1 , 3 3}[0,51-3,46] \quad \mathbf{0 , 5 6 3}$

$\mathbf{2 5 , 1}[17,2-36,6] \quad<\mathbf{0 , 0 0 1} \quad \mathbf{1 7 , 5} \quad[9,61-31,7] \quad<\mathbf{0 , 0 0 1}$

$\mathbf{3 1 , 5}[23,8-41,7] \quad<\mathbf{0 , 0 0 1} \quad \mathbf{2 4 , 2} \quad[17,7-33,1] \quad<\mathbf{0 , 0 0 1}$

$\mathbf{1 , 4 4}[1,12-1,84] \quad \mathbf{0 , 0 0 4} \quad \mathbf{1 , 8 8} \quad[1,40-2,53] \quad<\mathbf{0 , 0 0 1}$

$\mathbf{1 , 4 7} \quad[1,02-2,12] \quad 0,040$

$\begin{array}{ccc}\text { réf } & - & - \\ \mathbf{1 , 0 3} & {[0,70-1,49]} & 0,897\end{array}$

$\mathbf{1 7 , 5}[9,61-31,7]<\mathbf{0 , 0 0 1}$
$\mathbf{2 4 , 2}[17,7-33,1]<\mathbf{0 , 0 0 1}$
$\mathbf{1 , 8 8}[1,40-2,53]<\mathbf{0 , 0 0 1}$

$\mathbf{1 , 8 2}[1,24-2,69] \quad 0,002$

$1,78[1,05-3,01] \quad 0,031$

réf - -

réf - -

$\mathbf{0 , 7 9}[0,56-1,10] \quad 0,160$

$\mathbf{0 , 7 4}[0,48-1,13] \quad 0,161$

$\mathbf{0 , 5 4}[0,29-0,98] \quad 0,044$

$\mathbf{1 , 0 4}[0,60-1,76] \quad 0,918$

$\mathbf{2 , 5 9}[1,94-3,45] \quad<\mathbf{0 , 0 0 1}$

$\mathbf{1 , 1 5}[0,97-1,35] \quad \mathbf{0 , 1 0 0}$

$\mathbf{1 , 5 6}[1,22-2,00]<\mathbf{0 , 0 0 1}$

$\mathbf{1 , 5 2}[1,31-1,77]<\mathbf{0 , 0 0 1}$

\footnotetext{
${ }^{1} \mathrm{n}$ allant de 8971 à 9266 selon les variables.
} 
L'association globale entre le contexte de la conception et le petit poids de naissance constatée en univarié, avec un sur-risque significatif du groupe traitement par rapport au groupe infertile, persiste après ajustement sur les caractéristiques sociodémographiques et les facteurs médicaux, et disparaît totalement dès ajustement sur la gémellité (tableau 12). Ce résultat est confirmé par l'analyse restreinte aux singletons et aux singletons à terme, pour lesquels aucune association n'est retrouvée (tableau 12).

Tableau 12 : Odds Ratio de petit poids de naissance lié au contexte de la conception selon le modèle logistique et la population d'analyse

\begin{tabular}{ccccccccc}
\hline \multicolumn{2}{c}{ population totale } & & singletons & & \multicolumn{2}{c}{ singletons à terme } \\
\hline OR & IC à $95 \%$ & $p$ & OR & IC à $95 \%$ & $p$ & OR & IC à $95 \%$ & $p$ \\
\hline
\end{tabular}

analyse univariée

$$
\mathbf{p}<\mathbf{0 , 0 0 1} \quad \mathbf{p}=\mathbf{0 , 1 2 7}
$$

$\mathbf{p}=\mathbf{0 , 4 6 7}$

traitement vs fertile

$2,40 \quad[1,69-3,41]<0,001$

$1,28 \quad[0,81-2,03] \quad 0,289$

$\begin{array}{lll}1,44 & {[0,78-2,67]} & 0,242\end{array}$

infertile vs fertile

$1,31 \quad[0,92-1,88] \quad 0,139$

$1,43 \quad[0,98-2,10] \quad 0,063$

$1,16 \quad[0,68-1,98] \quad 0,590$

traitement vs infertile

$1,83 \quad[1,15-2,90] \quad 0,010$

$0,89 \quad[0,51-1,56] \quad 0,692$

$1,25 \quad[0,58-2,68] \quad 0,573$

modèle multivarié

1 : facteurs sociodémographiques

traitement vs fertile infertile vs fertile traitement vs infertile

2 : ... + facteurs médicaux traitement vs fertile infertile vs fertile traitement vs infertile

$3: \ldots+$ gémellité

traitement vs fertile infertile vs fertile traitement vs infertile

$4: \ldots+$ prématurité

traitement vs fertile infertile vs fertile traitement vs infertile

$$
\mathbf{p}=\mathbf{0 , 3 9 0}
$$$$
\mathbf{p}=\mathbf{0 , 3 5 4}
$$

$2,48 \quad[1,74-3,53]<0,001$

$1,39 \quad[0,85-2,27] \quad 0,194$

$1,58 \quad[0,81-3,08] \quad 0,175$

$1,10 \quad[0,75-1,62] \quad 0,632$

$1,14 \quad[0,75-1,72] \quad 0,545$

0,91

$[0,50-1,66]$

0,752

$2,26[1,40-3,64] \quad 0,001$

$1,22 \quad[0,67-2,22] \quad 0,518$

$1,75 \quad[0,75-4,05]$

0,194

$$
\begin{array}{ccc} 
& \multicolumn{2}{c}{\mathbf{p}<\mathbf{0 , 0 0 1}} \\
2,23 & {[1,56-3,18]} & <0,001 \\
1,00 & {[0,68-1,48]} & 0,996 \\
2,22 & {[1,38-3,59]} & 0,001
\end{array}
$$$$
\mathbf{p}=\mathbf{0 , 6 7 5}
$$$$
\mathbf{p}=\mathbf{0 , 3 9 2}
$$$$
1,25 \quad[0,76-2,05] \quad 0,375
$$$$
1,51
$$$$
[0,77-2,93] \quad 0,228
$$$$
1,03 \quad[0,67-1,57] \quad 0,897
$$$$
0,86
$$$$
\text { [0,47-1,57] } \quad 0,621
$$

$1,22 \quad[0,66-2,23] \quad 0,526$

$1,75 \quad[0,76-4,08]$

0,191

$$
\mathbf{p}=\mathbf{0 , 3 7 7}
$$

$0,71 \quad[0,39-1,29] \quad 0,258$

$0,79 \quad[0,49-1,27] \quad 0,332$

$0,90 \quad[0,44-1,83] \quad 0,766$ $\mathbf{p}=\mathbf{0 , 7 3 9}$

$0,98 \quad[0,53-1,82] \quad 0,954$

$0,82[0,50-1,35] \quad 0,438$

$1,19 \quad[0,58-2,47] \quad 0,635$ 


\subsubsection{Analyse en sous-groupes de traitement de l'infertilité}

Les mêmes analyses ont été conduites en comparant les groupes de traitement FIV (avec ou sans ICSI) et stimulation au groupe fertile.

Comme précédemment, les liens connus entre prématurité et niveau de diplôme maternel, statut monoparental et gémellité sont retrouvés après ajustement, mais pas l'association avec l'âge maternel, le diabète gestationnel ou le tabagisme au troisième trimestre de la grossesse (tableau A11).

Le traitement de l'infertilité est significativement associé à la prématurité après ajustement sur toutes les variables du modèle. Le sur-risque significatif du groupe FIV \pm ICSI par rapport au groupe stimulation observé en univarié persiste après ajustement sur les facteurs sociodémographiques et médicaux, mais disparaît après prise en compte de la gémellité, ce que confirme l'analyse restreinte aux singletons (tableau 13) : il existe une association significative entre traitement de l'infertilité et prématurité, sans différence significative entre les groupes FIV \pm ICSI et stimulation. 
Tableau 13 : Odds Ratio de prématurité lié au type de traitement de l'infertilité selon le modèle logistique et la population d'analyse

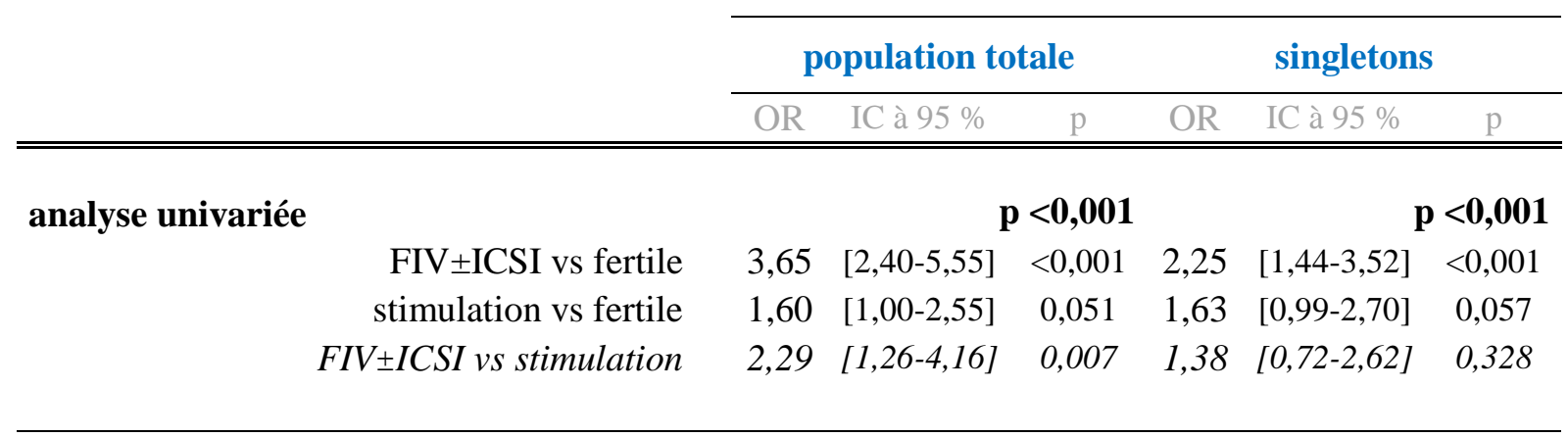

modèle multivarié

1 : facteurs sociodémographiques

FIV \pm ICSI vs fertile stimulation vs fertile FIV $\pm I C S I$ vs stimulation

2 : ... + facteurs médicaux

FIV \pm ICSI vs fertile stimulation vs fertile FIV \pm ICSI vs stimulation

$3: \ldots+$ gémellité

FIV \pm ICSI vs fertile stimulation vs fertile FIV \pm ICSI vs stimulation

\begin{tabular}{lllllll} 
& \multicolumn{3}{c}{$\mathbf{p}<\mathbf{0 , 0 0 1}$} & \multicolumn{3}{c}{$\mathbf{p}<\mathbf{0 , 0 0 1}$} \\
3,36 & {$[2,25-5,01]$} & $<0,001$ & 2,23 & {$[1,36-3,66]$} & 0,002 \\
1,87 & {$[1,17-3,01]$} & 0,009 & 1,90 & {$[1,15-3,16]$} & 0,013 \\
1,80 & {$[1,00-3,22]$} & 0,049 & 1,17 & {$[0,60-2,30]$} & 0,644
\end{tabular}

\begin{tabular}{cccccc} 
& \multicolumn{3}{c}{$\mathbf{p}<\mathbf{0 , 0 0 1}$} & \multicolumn{2}{r}{$\mathbf{p}<\mathbf{0 , 0 0 1}$} \\
3,52 & {$[2,35-5,26]$} & $<0,001$ & 2,41 & {$[1,46-3,95]$} & 0,001 \\
1,73 & {$[1,05-2,84]$} & 0,031 & 1,73 & {$[1,01-2,97]$} & 0,045 \\
2,03 & {$[1,11-3,73]$} & 0,022 & 1,39 & {$[0,69-2,80]$} & 0,359
\end{tabular}

$\mathbf{p}=\mathbf{0 , 0 2 4}$

$1,87 \quad[1,15-3,04] \quad 0,012$

$1,49 \quad[0,86-2,57] \quad 0,154$

$1,26[0,64-2,48] \quad 0,511$ 
De même, les liens connus du petit poids de naissance avec le niveau de diplôme maternel, la gémellité, la prématurité, la primiparité, l'IMC maternel avant la grossesse et le sexe féminin sont retrouvés après ajustement (tableau A11). L'association avec le tabagisme au troisième trimestre de la grossesse est ici faible mais significative, et l'association avec les revenus du foyer à la limite de la significativité.

Il n'y a pas de lien entre le traitement de l'infertilité et le petit poids de naissance après prise en compte de l'ensemble des variables du modèle. L'association globale constatée en univarié, avec un sur-risque du groupe FIV \pm ICSI par rapport au groupe stimulation à la limite de la significativité, persiste après ajustement sur les caractéristiques sociodémographiques et les facteurs médicaux mais disparaît totalement dès ajustement sur la gémellité (tableau 14). Ce résultat est confirmé par l'analyse restreinte aux singletons et aux singletons à terme, pour lesquels aucune association n'est retrouvée (tableau 14). 
Tableau 14 : Odds Ratio de petit poids de naissance lié au type de traitement de l'infertilité selon le modèle logistique et la population d'analyse

\begin{tabular}{ccccccccc}
\hline \multicolumn{2}{c}{ population totale } & & singletons & \multicolumn{3}{c}{ singletons à terme } \\
\hline OR & IC à $95 \%$ & $p$ & OR & IC à $95 \%$ & $p$ & OR & IC à $95 \%$ & $p$ \\
\hline
\end{tabular}

analyse univariée

$$
\mathbf{p}<\mathbf{0 , 0 0 1} \quad \mathbf{p}=\mathbf{0 , 5 8 8}
$$

$$
\mathbf{p}=\mathbf{0 , 4 9 7}
$$

FIV \pm ICSI vs fertile

$2,76 \quad[1,70-4,49] \quad<0,001$

$1,30 \quad[0,73-2,32] \quad 0,370$

$0,99 \quad[0,42-2,34] \quad 0,983$

stimulation vs fertile

$1,29[0,66-2,52] \quad 0,448$

$1,25 \quad[0,57-2,77] \quad 0,580$

$1,77 \quad[0,69-4,55] \quad 0,238$

FIV \pm ICSI vs stimulation

$2,13 \quad[0,96-4,75]$

0,064

1,04 [0,40-2,71]

0,936

$0,56 \quad[0,16-1,95]$

0,362

modèle multivarié

1 : facteurs sociodémographiques FIV \pm ICSI vs fertile

\begin{tabular}{llllllllll} 
& \multicolumn{3}{c}{$\mathbf{p}<\mathbf{0 , 0 0 1}$} & \multicolumn{3}{c}{$\mathbf{p}=\mathbf{0 , 4 9 7}$} & \multicolumn{3}{c}{$\mathbf{p}=\mathbf{0 , 4 5 8}$} \\
2,53 & {$[1,62-3,96]$} & $<0,001$ & 1,37 & {$[0,73-2,57]$} & 0,330 & 1,07 & {$[0,44-2,65]$} & 0,876 \\
1,43 & {$[0,71-2,88]$} & 0,318 & 1,38 & {$[0,59-3,23]$} & 0,464 & 1,94 & {$[0,68-5,51]$} & 0,212 \\
1,77 & {$[0,80-3,94]$} & 0,160 & 0,99 & {$[0,36-2,78]$} & 0,991 & 0,55 & {$[0,15-2,09]$} & 0,383
\end{tabular}

$2: \ldots+$ facteurs médicaux FIV \pm ICSI vs fertile stimulation vs fertile

\begin{tabular}{|c|c|c|}
\hline & & $=\mathbf{0 , 0 0}$ \\
\hline 2,32 & {$[1,48-3,62]$} & $<0,001$ \\
\hline 29 & {$[0,63-2,65]$} & 0,484 \\
\hline 79 & {$[0,79-4,05]$} & 0,161 \\
\hline & & $=0,6$ \\
\hline & {$[0,39-1,38]$} & 0,33 \\
\hline & {$[0,35-2,24]$} & 0,80 \\
\hline & {$[0,29-2,36]$} & 0,72 \\
\hline
\end{tabular}
FIV \pm ICSI vs stimulation

$4: \ldots+$ prématurité

$$
\mathbf{p}=\mathbf{0 , 2 1 8}
$$$$
\mathbf{p}=\mathbf{0 , 9 3 2}
$$

FIV \pm ICSI vs fertile

$0,52 \quad[0,24-1,10] \quad 0,088$

$0,72 \quad[0,24-2,18] \quad 0,564$

$0,90 \quad[0,44-1,84] \quad 0,771$

stimulation vs fertile

$0,72 \quad[0,20-2,53] \quad 0,606$

$0,85 \quad[0,26-2,82] \quad 0,796$

FIV \pm ICSI vs stimulation

$1,05 \quad[0,27-4,04]$

0,941 


\section{DISCUSSION}

\subsection{Résultats}

Le premier objectif de ce travail était de décrire les conditions entourant la naissance des enfants conçus avec une aide médicale et leur état de santé dans les deux premiers mois de vie. Les données Elfe recueillies en population générale ont permis d'inclure dans notre analyse les enfants issus de stimulation ovarienne, peu étudiés, les recherches dans ce domaine se limitant le plus souvent aux techniques d'AMP. La stimulation peut en effet être réalisée en dehors des centres d'AMP et échappe donc généralement aux évaluations.

Nous trouvons dans le groupe traité des foyers caractérisés par une situation sociodémographique très favorable en termes de niveau de diplôme, d'emploi, de revenus et de couverture sociale. Les mères ont eu avant et pendant leur grossesse un comportement de santé moins à risque et plus préventif. Elles ont malgré cela présenté davantage de pathologies de la grossesse et dû être plus souvent hospitalisées. Conformément à la littérature [2, 3, 69, 75-79], nous retrouvons des taux plus élevés de gémellité, de déclenchement, de césarienne, de prématurité, de petit poids de naissance et de transfert après la naissance chez les enfants conçus avec une aide médicale. Les anomalies congénitales n'ont pas été observées plus fréquemment dans cette population (2,4\% dans le groupe traitement vs 2,1\% dans le groupe infertile et 2,6\% dans le groupe fertile, $\mathrm{p}=0,711$ ), contrairement à d'autres études [3-7, 10], toutefois il faut rappeler que nous ne considérons que les naissances vivantes : les interruptions médicales de grossesse (IMG), les morts fœetales in utero spontanées et les enfants morts-nés ne sont pas pris en compte et pourraient fausser ce résultat s'ils étaient plus nombreux après un traitement de l'infertilité. L'InVS estimait à 27000 le nombre de foetus porteurs d'anomalies congénitales en 2012 en France dont environ 20000 nouveau-nés vivants, 440 mort-nés et 6700 IMG, soit une prévalence totale de 3,3\% [95] légèrement supérieure à celle observée ici. De plus, l'inclusion 
des enfants dans Elfe reposait sur le volontariat des familles : les mères d'enfant porteur d'une anomalie congénitale étaient peut-être plus susceptibles de refuser l'enquête ; cependant rien n'indique que ce serait davantage le cas dans un contexte de traitement de l'infertilité. Il ne semble pas y avoir non plus de différence de recours hospitalier dans les deux premiers mois de vie. Les mères du groupe traitement tendent à déclarer davantage de problèmes de santé, ce qui pourrait traduire une vigilance plus grande plutôt qu'un réel sur-risque. Il a en effet été décrit dans la littérature un niveau d'anxiété plus important sur la survie du fœtus pendant la grossesse, puis du nouveau-né après la naissance, chez les couples ayant eu recours à une aide médicale pour concevoir, ainsi qu'une estime de soi et une confiance en ses capacités plus faibles qu'en cas de conception spontanée ; la persistance, plusieurs mois après la naissance, de l'inquiétude quant au devenir de l'enfant pourrait être liée à une idéalisation de la parentalité pendant la grossesse, qui rendrait plus difficile la transition vers une parentalité assurée et confiante dans son identité [96-99].

La taille de la cohorte a rendu possible une analyse en sous-groupes de traitement de l'infertilité, objectif secondaire de ce travail, en particulier la comparaison des groupes FIV \pm ICSI et stimulation (tableaux A6 à A10). Leurs caractéristiques sociodémographiques sont similaires, hormis la part des mères de plus de 35 ans $(39,7 \%$ en FIV \pm ICSI vs 22,8 \%, $\mathrm{p}<0,001)$. Les mères diffèrent peu par leurs antécédents, mais présentent plus de placenta prævia hémorragique en FIV \pm ICSI $(2,7 \%$ vs $0,3 \%, p=0,016)$. Les taux de gémellité et de prématurité sont significativement plus élevés en FIV \pm ICSI $(16,1 \%$ vs 4,9\%, p<0,001 et $13,6 \%$ vs $6,4 \%, \mathrm{p}=0,006$, respectivement) celui de petit poids de naissance tend à l'être $(10,4 \%$ vs 5,2\%,p=0,059). Dans une large étude américaine comparant les complications néonatales chez des enfants conçus après AMP, après stimulation ovarienne ou naturellement, D'Angelo et al. observaient également davantage de gémellité après FIV qu'après stimulation $(22,1 \%[18,2-26,0]$ vs $5,9 \%[4,3-7,5] ; 1,2 \%[1,0$ - 1,4] après conception naturelle), davantage de prématurité $(30,1 \%[25,7$ - 34,5] vs $15,0 \%$ [12,2 - 17,7] ; 8,6 \% [8,1 - 9,2] après 
conception naturelle), et davantage de petit poids de naissance $(21,9 \%[19,2-25,1]$ vs $10,6 \%$ $[9,0-12,3] ; 5,7 \%[5,9-5,5]$ après conception naturelle) $[100]$.

Le deuxième objectif de ce travail était d'explorer les facteurs jouant un rôle dans les complications pouvant survenir après une conception obtenue grâce à un traitement de l'infertilité. L'originalité de ce projet est d'explorer la problématique des enfants conçus avec une aide médicale sur une large cohorte pluridisciplinaire et à visée représentative au niveau national [86]. La cohérence de nos données avec celles de l'enquête nationale périnatale et de l'agence de biomédecine apporte des éléments rassurants sur la qualité de l'échantillon Elfe. Une telle enquête permet une description fine des informations sociodémographiques des enfants nés après traitement, aspect rarement décrit dans la littérature. Les centres d'AMP ne collectent pas ce type de renseignements, ou de manière très partielle et non standardisée, aussi les recherches françaises apportent-elles peu de données sur le sujet. Cette approche rend possible l'étude de la prématurité et du petit poids de naissance chez les enfants conçus avec une aide médicale en incluant ces variables sociales pour la première fois, alors qu'il s'agit de facteurs de risque connus en dehors du cadre de l'AMP [72, 74].

Après ajustement sur les facteurs sociodémographiques, les facteurs médicaux et la gémellité, il existe une association significative entre le contexte de la conception (après traitement, après période d'infertilité, sans période d'infertilité) et la prématurité, sans différence significative entre les groupes traitement et infertile (OR de 1,53 et 1,52 respectivement par rapport au groupe fertile, $\mathrm{p}=0,977)$. L'OR retrouvé en univarié n'est pas modifié par l'ajustement sociodémographique, ce qui suggère que l'effet du traitement ne passe pas par cette voie. L'ajustement sur la gémellité quant à lui ne fait pas disparaître l'association globale mais annule la différence entre les groupes traitement et infertile : une prématurité plus fréquente est retrouvée, non liée exclusivement au plus fort taux de grossesse multiple, comme dans la 
littérature [75-79], mais ce sur-risque semblerait lié aux caractéristiques des couples infertiles plutôt qu'au traitement. La même analyse effectuée en sous-groupes de traitement retrouve l'association traitement-prématurité, sans mettre en évidence de différence significative entre les groupes FIV \pm ICSI et stimulation (OR de 1,87 et 1,49 respectivement par rapport au groupe fertile, $\mathrm{p}=0,511)$. Hayashi et al. ont comparé les complications néonatales chez des singletons conçus après stimulation ovarienne, insémination artificielle ou FIV (avec appariemment de chacun de ces trois groupes à un groupe contrôle de singletons conçus naturellement) dans une très large cohorte japonaise comptant plusieurs milliers d'enfants; ils ont observé une prématurité plus fréquente dans les trois groupes de traitement de l'infertilité, avec des taux similaires quelle que soit la technique utilisée [101]. Les auteurs en concluaient que les facteurs maternels associés à l'infertilité pourraient contribuer aux complications néonatales davantage que le traitement de l'infertilité lui-même.

Aucune association n'est retrouvée en revanche entre contexte de la conception et petit poids de naissance, ni entre type de traitement et petit poids de naissance, le sur-risque observé en analyse univariée disparaissant totalement après ajustement sur la gémellité : la part de la prématurité non liée à la gémellité évoquée au paragraphe précédent n'apparaît plus clairement sur le petit poids de naissance. Par ailleurs, chez les singletons et les singletons à terme, l'OR lié au contexte de la conception ou au type de traitement n'est significatif ni en univarié ni en multivarié. Plusieurs études ont pourtant rapporté un sur-risque d'hypotrophie chez les singletons issus d'AMP [77], et de petit poids pour l'âge gestationnel [6]. Zhu et al., dans une étude de cohorte danoise, en utilisant une approche en trois groupes (conception après traitement, après période d'infertilité, sans période d'infertilité), ont démontré un sur-risque de petit poids pour l'âge gestationnel chez les couples infertiles, sans différence significative selon qu'ils aient ou non suivi un traitement (OR de 1,24 [1,10-1,40] chez les non traités, OR de 1,40 [1,23 - 1,60] chez les traités) [27]. Hayashi et al. ont observé dans l'étude précédemment citée un petit poids de naissance plus fréquent dans les trois groupes de traitement de 
l'infertilité, avec des taux similaires quelle que soit la technique utilisée [101]. Peut-être notre analyse manque-t-elle de puissance (nos effectifs sont cinq à dix fois plus faibles que ceux de Zhu et al. et Hayashi et al.), ce qui empêcherait de mettre en évidence un lien résiduel entre le contexte de la conception et le petit poids. Il serait par ailleurs intéressant d'utiliser des modèles rendant compte de manière plus complète des relations entre variables et permettant de mieux faire la part entre effet direct et facteur intermédiaire. C'est le cas des modèles par analyse de cheminement (pathway analysis), non mis en œuvre ici. Cette approche récente, décrite par Erikson et Buis [102, 103], permettrait de décomposer l'effet total du traitement de l'infertilité en un effet indirect (médié par la gémellité / la prématurité) et un effet direct. Une difficulté serait de prendre en compte la pondération (la commande correspondante ldecomp implémentée dans Stata ne s'applique pas aux données pondérées) ; il faudrait éventuellement essayer cette méthode sans pondérer et en contrôlant dans le modèle pour les variables utilisées dans la pondération. 


\subsection{Limites et révision des objectifs}

En premier lieu, rappelons que tous les résultats présentés dans ce travail sont provisoires et qu'ils devront être recalculés avec la pondération mise à jour (publiée en juillet 2015 et corrigeant l'effet de saisonnalité) et en tenant compte du plan de sondage pour une estimation plus exacte de la variance.

Cette étude comporte d'autres limites. Tout d'abord, la cohorte Elfe n'a pas été élaborée dans le but d'étudier spécifiquement les enfants conçus avec une aide médicale. Ce travail n'a ainsi pas pu inclure les enfants grands prématurés (nés avant 33 SA), population reconnue comme étant la plus à risque de morbi-mortalité [71]; une coopération est cependant prévue entre les équipes Elfe et Épipage 2, et permettra plus tard d'inclure également ces enfants dans l'analyse. Les questionnaires n'apportent pas d'information sur les indications du traitement, les causes de l'infertilité du couple, le nombre d'embryons transférés, l'existence d'une réduction embryonnaire, le centre d'AMP, alors que ces différents facteurs peuvent avoir un retentissement sur la grossesse et que les pratiques sont encore très centre-dépendantes [65].

Nous avons observé dans l'analyse descriptive des différences significatives entre les enfants issus de FIV classiques et ceux issus de FIV avec ICSI (tableaux A6 à A10); par exemple, le taux de petit poids de naissance est ici significativement plus élevé dans le groupe FIV classique qu'en FIV avec ICSI, avec un taux de transfert après la naissance plus important. Ces différences ne vont pas dans le sens attendu (plus un traitement est invasif plus on s'attend à ce qu'il ait un impact), mais certaines ont déjà été rapportées dans quelques études [104], dont une large cohorte norvégienne [105]. Une hypothèse expliquant ces effets spécifiques dans le groupe FIV classique par rapport au groupe FIV avec ICSI serait qu'il existe un effet de sélection d'une population spécifique, plutôt qu'un effet du type de traitement, les FIV 
classiques étant désormais plutôt réservées aux cas d'infertilité d'origine féminine et les FIV avec ICSI aux cas d'infertilité d'origine masculine. Dans notre étude, les mères du groupe FIV classique tendent ainsi à être davantage fumeuses et hypertendues (tableau A7), deux facteurs pouvant être associés d'une part au fait que la femme n'arrive pas à obtenir de grossesse et d'autre part au petit poids de l'enfant à la naissance et à son transfert. Les informations disponibles sur les traitements et sur les antécédents médicaux maternels sont cependant trop succinctes pour explorer et expliquer de possibles différences entre les enfants nés après FIV classique ou après FIV avec ICSI. Par ailleurs, ces différences restent ici limitées à quelques indicateurs, la majorité ayant des valeurs très proches. Au final, il a donc été décidé de regrouper les deux techniques dans un même ensemble (FIV avec ou sans ICSI) pour l'analyse multivariée.

Ensuite, l'exploration des données de santé à deux mois s'est heurtée à la faiblesse d'informations basées uniquement sur les déclarations maternelles : les items du questionnaire manquaient de spécificité et ne permettaient d'appréhender l'état de santé des enfants qu'au travers de préoccupations rapportées par la mère, très diverses dans leur nature et leur gravité, et très fréquentes à cet âge donc peu discriminantes. Le recours aux urgences, plus objectif, est ici à interpréter avec prudence, car reconstitué à partir de plusieurs questions, qui devaient initialement être posées aux mères en face-à-face mais qui l'ont finalement été par enquête téléphonique. La question relative à une hospitalisation de l'enfant au cours des deux premiers mois de vie était elle sans ambiguïté. La prise de poids dans les deux premiers mois, variable $a$ priori la plus pertinente, n'était complète dans la base que dans $51,7 \%$ des cas, l'âge lors de la mesure et/ou la valeur du poids étaient des données manquantes chez 48,3\% des enfants. Cette analyse a permis d'alerter l'équipe Elfe sur la qualité de cette variable et de mettre à jour un problème de production du fichier de données Elfe au niveau du prestataire chargé de l'enquête. 
Les données vont pouvoir être récupérées (car elles ont bien été collectées) et ré-introduites dans la base, ce qui permettra d'explorer cet indicateur dans une future analyse.

Enfin, la distinction de la part des effets du traitement et de l'infertilité grâce à la constitution de deux groupes de comparaison (infertile et fertile) a soulevé des difficultés non anticipées. La construction du groupe infertile à partir du questionnaire a en effet nécessité trois essais successifs (tableau 15) conduisant à un resserrement de la définition de cette population. Le délai de conception ( $\geq 1$ an) a d'abord été utilisé comme seul critère d'infertilité (groupe infertile v1). Le risque était de regrouper des mères infertiles et des mères non planificatrices, sans contraception ni réel projet d'enfant, type de comportement très lié à un niveau social bas. Un fort gradient sociodémographique AMP > fertile > infertile a de fait été retrouvé. Il a donc été décidé de restreindre la population infertile aux grossesses déclarées désirées par les deux parents (groupe infertile v2) puis aux cas où il y a eu "consultation pour rendre la grossesse possible" sans traitement ultérieur (groupe infertile v3), afin d'éliminer le "bruit" des grossesses non réellement planifiées. Malgré cela, le gradient social a persisté en partie, avec un niveau sociodémographique du groupe infertile plus faible que dans les deux autres groupes. 


\section{Tableau 15 : Caractéristiques sociodémographiques des différents groupes infertiles considérés}

\begin{tabular}{ccccc}
\hline $\begin{array}{c}\text { traitement } \\
(\mathrm{N}=790)\end{array}$ & fertile & infertile v3 & infertile v2 & infertile v1 \\
$\%$ & $(\mathrm{~N}=7661)$ & $(\mathrm{N}=1044)$ & $(\mathrm{N}=2605)$ & $(\mathrm{N}=3055)$ \\
$\%$ & $\%$ & $\%$ & $\%$ & $\%$ \\
\hline
\end{tabular}

mère française de naissance

diplôme le plus élevé obtenu par la mère

$\leq$ brevet des collèges

$C A P / B E P$

baccalauréat

$b a c+2$

$>b a c+2$

emploi lors de la grossesse

couverture maladie maternelle

$C M U$ / AME / aucun régime

régime sans complémentaire

régime et complémentaire

ressources mensuelles du foyer

$$
\begin{array}{r}
<2500 \text { euros } \\
2500-3999 \text { euros } \\
\geq 4000 \text { euros }
\end{array}
$$

vie en couple à la naissance
89,9

91,0

81,7

81,8

80,9

$4,2 \quad 7,1$

9,9

12,4

14,6

$11,2 \quad 13,7$

17,8

20,6

20,8

13,8

17,6

23,1

38,6

18,6

19,6

19,7

22,1

19,9

19,0

31,6

27,5

25,9

88,9

80,0

74,4

70,9

69,4

\section{2,5}

4,9

5,5

4,8

89,6

9,2

10,6

13,1

6,6

7,0

7,4

84,2

82,4

79,4

34,4

37,7

41,1

42,3

40,8

39,0

23,3

21,5

19,9

37,2

25,2

99,9

98,9

98,2

93,3

Quelques autres résultats interrogent sur la cohérence du groupe infertile et sa pertinence comme témoin du groupe traitement : une plus forte proportion de grossesse antérieure que le groupe fertile, à mettre en relation avec un fort taux de fausse couche dans la période précédant la conception de l'enfant inclus dans l'étude, un comportement à risque ou préventif pendant la grossesse qui ne va pas toujours dans le même sens, un fort taux de césarienne, un score d'Apgar significativement plus bas que dans les deux autres groupes, un risque maximal ou intermédiaire selon les variables.

L'analyse descriptive a été poursuivie en ajustant sur quelques variables sociodémographiques (âge, nationalité, niveau d'études, couverture maladie, situation de couple de la mère), et en considérant d'autres définitions des sujets infertiles (infertilité légère 
avec un temps de conception de 6 à 12 mois, restriction aux primigestes afin d'exclure les cas de fausses couches répétées qui pourraient relever d'un type de traitement spécifique, exclusion des mères étrangères et de bas niveau de diplôme...), sans permettre de mieux cerner le groupe infertile (résultats non présentés).

Deux hypothèses peuvent être formulées. Le groupe infertile est composé d'un mélange de deux populations, dont la population infertile qu'on souhaite isoler. Ou bien, les couples infertiles mais qui ne recourent pas à une assistance médicale constituent effectivement un groupe particulier, du fait de caractéristiques sociodémographiques différentes, et/ou de différences dans le comportement de recours aux soins, et/ou de différences dans le rapport à la planification de la grossesse, voire d'une d'infertilité différente ne nécessitant pas le même type de traitement. Par ailleurs, ce groupe, s'il est particulier, risque de ne pas être pris en charge de la même manière par le corps médical (l'AMP n'est pas un traitement vital et nécessite une forte adhésion des parents).

Quelle que soit l'hypothèse retenue, ces résultats montrent que le groupe témoin infertile diffère du groupe traitement autrement que par le traitement ou un niveau d'infertilité plus faible, sans qu'il soit possible ici de déterminer les liens entre le gradient socio-démographique constaté et le recours à un traitement de l'infertilité, ou le type d'infertilité. On ne peut donc exclure totalement une spécificité de la population infertile non traitée par rapport à la population traitée qui expliquerait un sur-risque équivalent entre ces deux groupes autrement que par un «non-effet» du traitement de l'infertilité per se. Même si le fait que le sur-risque soit aussi équivalent entre différentes techniques (ici, FIV et stimulation) est également un argument en défaveur d'un rôle prépondérant du traitement de l'infertilité.

La piste du groupe témoin infertile est présente dans la littérature, mais peu de résultats ont été publiés pour l'instant. Zhu et al. ont limité leur définition du groupe infertile à un délai 
nécessaire pour concevoir supérieur à 12 mois sans contraception, et ont exlu les grossesses non planifiées [25-28]. Différents facteurs de confusion étaient décrits, mais aucun facteur sociodémographique. Cooper et al., dans leur comparaison d'enfants de couples infertiles non traités avec des enfants de couples traités par stimulation, de couples traités par FIV, et de couples fertiles, n'avaient comme seul critère d'infertilité que le délai nécessaire pour concevoir supérieur à 12 mois [29]. Là encore, les auteurs n’ont pas rapporté les caractéristiques sociodémographiques des couples. En revanche, ils ont décrit les causes d'infertilité, celle-ci étant plus fréquemment inexpliquée chez les couples non traités. Les deux équipes concluaient que l'infertilité, ou plus probablement ses déterminants et les processus pathologiques qui lui sont associés, pouvaient avoir un impact plus important que le traitement de l'infertilité sur le petit poids de naissance [27-29]. 


\subsection{Perspectives de recherche}

Nous avons dans ce travail conservé et analysé le groupe infertile jusqu'au terme de nos objectifs, ce qui a soulevé des questions quant à la nature de ce groupe non abordées auparavant dans la littérature. Les critères de sélection des couples infertiles étaient aussi précis voire plus restreints que dans la littérature, ce qui devrait éloigner le risque d'une mauvaise spécification de notre groupe d'étude. Une description plus approfondie de ses caractéristiques (diagnostic étiologique de l'infertilité, autres antécédents médicaux...) est nécessaire pour pouvoir exclure une spécificité de la population infertile non traitée par rapport à la population traitée, qui expliquerait un sur-risque équivalent entre ces deux groupes autrement que par un «non-effet » du traitement de l'infertilité per se. Une solution alternative pour distinguer effet du traitement et effet de l'infertilité pour la suite du projet est la comparaison FIV \pm ICSI / stimulation, avec la même hypothèse de parents de plus en plus infertiles soumis à un traitement de plus en plus invasif. Les effectifs et la puissance ne seront bien sûr pas les mêmes.

L'étude du devenir à plus long terme des enfants conçus avec une aide médicale, en particulier leur développement psychomoteur et pondéral entre la naissance et 3 ans, bénéficiera de la description fine du contexte sociodémographique qui fait l'originalité de nos résultats, et d'indicateurs de développement du jeune enfant mesurés cette fois à partir d'outils validés :

- le Child Development Inventory (CDI) à 1 an : questionnaire parental permettant d'évaluer le développement de la socialisation, de l'autonomie, de la motricité (globale ou fine), du langage expressif et réceptif ... chez l'enfant de moins de 6 ans $[106,107]$.

- le compte-rendu parental de Mac Arthur à 2 ans: outil d'évaluation précoce retraçant le développement langagier de l'enfant entre 8 et 30 mois, des premiers 
gestes non verbaux jusqu'au premier vocabulaire et le début d'acquisition de la grammaire, grâce à un questionnaire auprès des parents [108].

- la sous-échelle Picture similarities du British Ability Scales (BAS, échelle anglaise de capacités) à 3 ans : le BAS est un ensemble de tests administrés à l'enfant permettant d'évaluer les capacités cognitives de l'enfant entre 30 mois et 18 ans. La sous-échelle Picture similarities (similarité d'images) teste le raisonnement sur les images et permet d'évaluer le développement cognitif non verbal $[109,110]$. 


\section{CONCLUSION}

Nous avons montré dans cette étude une augmentation significative du risque de prématurité chez les enfants de couples infertiles, traités ou non traités, par rapport aux enfants de couples fertiles, après ajustement sur plusieurs facteurs ; il n'y a pas en revanche de différence entre couples infertiles traités et non traités, la différence observée avant ajustement semblant complètement liée à la gémellité associée au traitement de l'infertilité, sans effet direct du traitement sur la prématurité. De même, il existe un sur-risque de prématurité après FIV \pm ICSI ou stimulation ovarienne par rapport au groupe fertile, sans différence entre ces deux groupes de traitement. Ces éléments sont en faveur d'un sur-risque de complications néonatales chez les enfants de parents infertiles, conçus grâce à un traitement ou non.

Ces résultats ont d'autant plus de valeur qu'ils tiennent compte des caractéristiques sociodémographiques parentales, facteurs de risque de prématurité connus. Nos résultats restent cependant préliminaires. Des caractéristiques différentes du groupe témoin "infertile sans traitement" par rapport au groupe "traitement", sociodémographiques notamment, incitent en effet à la prudence et ne permettent pas d'exclure totalement un effet des techniques médicales utilisées. Il sera intéressant dans l'avenir d'étudier plus spécifiquement la population infertile qui ne recourt pas à un traitement médical. Par ailleurs, les analyses réalisées dans ce travail seront approfondies par des analyses de cheminement modélisant de manière plus complète les relations entre variables, en particulier les effets de médiation.

Les résultats présentés ici s'inscrivent dans le cadre plus large d'un projet d'étude du devenir des enfants conçus avec une aide médicale. L'intérêt de la description fine du contexte sociodémographique de ces enfants, qui fait l'originalité de ce travail, prendra tout son sens à plus long terme dans leur suivi, en particulier avec l'analyse du développement psychomoteur et pondéral entre la naissance et 3 ans. 


\section{BIBLIOGRAPHIE}

1. La Rochebrochard (de), E., 200000 enfants conçus par fécondation in vitro en France depuis 30 ans. Population et Sociétés, 2008. 1-4.

2. Blondel, B., Kermarec, M., Enquête Nationale Périnatale 2010. Les naissances en 2010 et leur évolution en 2003. 2011, Rapport Inserm - DGS - DREES: Paris.

3. Bouillon, C., Fauque, P., Devenir des enfants issus des techniques d'assistance médicale à la procréation. Arch Pediatr, 2013. 20(5): p. 575-9.

4. Hansen, M., et al., The risk of major birth defects after intracytoplasmic sperm injection and in vitro fertilization. $\mathrm{N}$ Engl J Med, 2002. 346(10): p. 725-30.

5. Hansen, M., et al., Assisted reproductive technologies and the risk of birth defects--a systematic review. Hum Reprod, 2005. 20(2): p. 328-38.

6. Hansen, M., Bower, C., Impact of assisted reproductive technologies on intrauterine growth and birth defects in singletons. Semin Fetal Neonatal Med, 2014.

7. Tararbit, K., et al., The risk for four specific congenital heart defects associated with assisted reproductive techniques: a population-based evaluation. Hum Reprod, 2013. 28(2): p. 367-74.

8. Kallen, B., et al., Cancer risk in children and young adults conceived by in vitro fertilization. Pediatrics, 2010. 126(2): p. 270-6.

9. Petridou, E.T., et al., In vitro fertilization and risk of childhood leukemia in Greece and Sweden. Pediatr Blood Cancer, 2012. 58(6): p. 930-6.

10. Rudant, J., et al., Fertility treatments, congenital malformations, fetal loss, and childhood acute leukemia: the ESCALE study (SFCE). Pediatr Blood Cancer, 2013. 60(2): p. 301-8.

11. Ceelen, M., et al., Growth and development of children born after in vitro fertilization. Fertil Steril, 2008. 90(5): p. 1662-73.

12. Leslie, G.I., et al., Children conceived using ICSI do not have an increased risk of delayed mental development at 5 years of age. Hum Reprod, 2003. 18(10): p. 206772.

13. Ponjaert-Kristoffersen, I., et al., Psychological follow-up study of 5-year-old ICSI children. Hum Reprod, 2004. 19(12): p. 2791-7.

14. Bay, B., Fertility treatment: long-term growth and mental development of the children. Dan Med J, 2014. 61(10): p. B4947.

15. Bay, B., et al., Fertility treatment and child intelligence, attention, and executive functions in 5-year-old singletons: a cohort study. Bjog, 2014. 121(13): p. 1642-51.

16. Kettner, L.O., et al., Assisted reproductive technology and somatic morbidity in childhood: a systematic review. Fertil Steril, 2015. 103(3): p. 707-19.

17. Pontesilli, M., et al., Subfertility and assisted reproduction techniques are associated with poorer cardiometabolic profiles in childhood. Reprod Biomed Online, 2015. 30(3): p. 258-67. 
18. Kondapalli, L.A., Perales-Puchalt, A., Low birth weight: is it related to assisted reproductive technology or underlying infertility? Fertil Steril, 2013. 99(2): p. 303-10.

19. Draper, E.S., et al., Assessment of separate contributions to perinatal mortality of infertility history and treatment: a case-control analysis. Lancet, 1999. 353(9166): p. 1746-9.

20. Basso, O., Baird, D.D., Infertility and preterm delivery, birthweight, and Caesarean section: a study within the Danish National Birth Cohort. Hum Reprod, 2003. 18(11): p. 2478-84.

21. Thomson, F., et al., Obstetric outcome in women with subfertility. Bjog, 2005. 112(5): p. 632-7.

22. Kapiteijn, K., et al., Does subfertility explain the risk of poor perinatal outcome after IVF and ovarian hyperstimulation? Hum Reprod, 2006. 21(12): p. 3228-34.

23. Jaques, A.M., et al., Adverse obstetric and perinatal outcomes in subfertile women conceiving without assisted reproductive technologies. Fertil Steril, 2010. 94(7): p. 2674-9.

24. Romundstad, L.B., et al., Effects of technology or maternal factors on perinatal outcome after assisted fertilisation: a population-based cohort study. Lancet, 2008. 372(9640): p. 737-43.

25. Zhu, J.L., et al., Infertility, infertility treatment, and congenital malformations: Danish national birth cohort. Bmj, 2006. 333(7570): p. 679.

26. Zhu, J.L., et al., Infertility, infertility treatment and twinning: the Danish National Birth Cohort. Hum Reprod, 2007. 22(4): p. 1086-90.

27. Zhu, J.L., et al., Infertility, infertility treatment, and fetal growth restriction. Obstet Gynecol, 2007. 110(6): p. 1326-34.

28. Zhu, J.L., et al., Infertility, infertility treatment and psychomotor development: the Danish National Birth Cohort. Paediatr Perinat Epidemiol, 2009. 23(2): p. 98-106.

29. Cooper, A.R., et al., Smaller fetal size in singletons after infertility therapies: the influence of technology and the underlying infertility. Fertil Steril, 2011. 96(5): p. 1100-6.

30. Hvidtjorn, D., et al., Risk of autism spectrum disorders in children born after assisted conception: a population-based follow-up study. J Epidemiol Community Health, 2011. 65(6): p. 497-502.

31. Hart, R., Norman, R.J., The longer-term health outcomes for children born as a result of IVF treatment. Part II--Mental health and development outcomes. Hum Reprod Update, 2013. 19(3): p. 244-50.

32. De Mouzon, J., Bachelot, A., Données de l'enquête caractéristiques des couples demandant une fécondation In Vitro en France, 2007, INED, Documents de travail, $\mathrm{n}^{\circ} 142$.

33. Dearden, L., et al., The socio-economic gradient in early child outcomes : evidence from the Millennium Cohort Study. Longitudinal and Life Course Studies, 2011. 2: p. $19-40$.

34. Hediger, M.L., et al., Assisted reproductive technologies and children's neurodevelopmental outcomes. Fertil Steril, 2013. 99(2): p. 311-7. 
35. Leridon, H., Fécondité, fertilité, stérilité : problèmes de terminologie, in Les Colloques de l'INSERM : facteurs de la fertilité humaine, INSERM, Editor. 1981. p. 17-30.

36. Schwartz, D., Importance de la durée d'infécondité dans l'appréciation de la fertilité d'un couple. Population, 1981. 2: p. 237-250.

37. Spira A., et al., Les troubles de la fertilité : état des connaissances et pistes pour la recherche, Rapport du groupe de travail réuni par l'Institut national de la santé et de la recherche médicale et par l'Agence de la biomédecine à la demande du Parlement (Article 51 de la loi $n^{\circ} 2011-814$ du 7 juillet 2011). 2012, INSERM, agence de la biomédecine.

38. Slama R., et al., La fertilité des couples en France. Bulletin Épidémiologique Hebdomadaire, 2012. 7-8-9: p. 87-91.

39. Blondel, B., et al., Trends in perinatal health in metropolitan France between 1995 and 2003: results from the National Perinatal Surveys. J Gynecol Obstet Biol Reprod (Paris), 2006. 35(4): p. 373-87.

40. Ohannessian, A., et al., Épidémiologie de la fertilité. EMC - Gynécologie, 2014. 9 (2): p. 1-7.

41. La Rochebrochard (de), E., Prioux, F., Des enfants toujours plus tard? Pg. 171-175, In: Dictionnaire de démographie et des sciences de la population, Meslé F -

Toulemon L - Véron J (editors). Armand Colin (Paris). 2011.

42. Baird, D.T., et al. ; ESHRE Capri Workshop Group, Fertility and ageing. Human Reproduction Update, 2005. 11 (3): p. 261-276.

43. De La Rochebrochard, E., Thonneau, P., Paternal age and maternal age are risk factors for miscarriage; results of a multicentre European study. Human Reproduction, 2002: p. 17(6):1649-1656.

44. De La Rochebrochard, E., et al., Paternal age over 40 years: the "amber light" in the reproductive life of men? J Androl, 2003. 24(4): p. 459-65.

45. Nybo Andersen, A.M., et al., Advanced paternal age and risk of fetal death: a cohort study. Am J Epidemiol, 2004. 160(12): p. 1214-22.

46. Carlsen, E., et al., Evidence for decreasing quality of semen during past 50 years. Bmj, 1992. 305(6854): p. 609-13.

47. Andersen, A.G., et al., High frequency of sub-optimal semen quality in an unselected population of young men. Hum Reprod, 2000. 15(2): p. 366-72.

48. Bujan, L., et al., Évolution temporelle et géographique des caractéristiques du sperme en France et dans le monde. Quo vadis ? . Bull Epidémiol Hebd, 2012. 7-8-9: p. 91-7.

49. Le Moal, J., et al., Evolution de la concentration spermatique en France entre 1989 et 2005 à partir des données de la base Fivnat. Bull Epidémiol Hebd, 2012. 7-8-9: p. 98102.

50. Adami, H.O., et al., Testicular cancer in nine northern European countries. Int J Cancer, 1994. 59(1): p. 33-8.

51. Purdue, M.P., et al., International patterns and trends in testis cancer incidence. Int J Cancer, 2005. 115(5): p. 822-7. 
52. Huyghe, E., et al., Testicular cancer variations in time and space in Europe. Eur Urol, 2007. 51(3): p. 621-8.

53. Chia, V.M., et al., International trends in the incidence of testicular cancer, 19732002. Cancer Epidemiol Biomarkers Prev, 2010. 19(5): p. 1151-9.

54. Kudjawu, Y., et al., Évolution nationale et variations régionales du taux de patients opérés pour cancer du testicule en France, 1998-2008. Bull Epidémiol Hebd, 2012. 78-9: p. 106-10.

55. Huyghe, E., et al., Fertility after testicular cancer treatments: results of a large multicenter study. Cancer, 2004. 100(4): p. 732-7.

56. Walschaerts, M., et al., Fertilité et cancer du testicule : une revue de la littérature. Bull Epidémiol Hebd, 2012. 7-8-9: p. 110-14.

57. Paulozzi, L.J., International trends in rates of hypospadias and cryptorchidism. Environ Health Perspect, 1999. 107(4): p. 297-302.

58. Skakkebaek, N.E., et al., Testicular dysgenesis syndrome: an increasingly common developmental disorder with environmental aspects. Hum Reprod, 2001. 16(5): p. 972-8.

59. Thonneau, P.F., et al., Cryptorchidism: incidence, risk factors, and potential role of environment; an update. J Androl, 2003. 24(2): p. 155-62.

60. Suzan, F., et al., Cryptorchidies et hypospadias opérés en France chez le garçon de moins de 7 ans (1998-2008). Bull Epidémiol Hebd, 2012. 7-8-9: p. 103-6.

61. Desdoits-Lethimonier, C., et al., Human testis steroidogenesis is inhibited by phthalates. Hum Reprod, 2012. 27(5): p. 1451-9.

62. L'assistance médicale à la procréation. 2013; Dossier Inserm réalisé en collaboration avec Michael Grynberg.

63. Code de la santé publique, lois de bioéthique du 6 août 2004 et du 7 juillet 2011, articles L. 2141-1 à 2142-4 relatifs à l'assistance médicale à la procréation.

64. Vayena, E., Rowe, P.J., Griffin, P.D., Current practices and controversies in assisted reproduction. Report of a meeting on "Medical, ethical and social aspects of assisted reproduction" held at WHO headquaters in Geneva, Switzerland, 17-21 September 2001, 2002, World Health Organization (Geneva): 396 pages.

65. Agence de la biomédecine, Rapport d'activité annuel d'AMP 2011. 2012.

66. Agence de la biomédecine, Rapport d'activité annuel d'AMP 2012. 2013.

67. La Rochebrochard (de), E., Des hommes médicalement assistés pour procréer: IAD, FIV, ICSI, bilan d'une révolution dans la prise en charge médicale de l'infertilité masculine. Population, 2003. 58(4-5):549-586.

68. Insee. statistiques de l'état civil et estimations de population.

69. Kupka, M.S., Ferraretti, A.P., et al., Assisted reproductive technology in Europe, 2010: results generated from European registers by ESHRE.Hum Reprod, 2014. 29(10): p. 2099-2113.

70. Senat, M.V., Ancel, P.Y., et al., How does multiple pregnancy affect maternal mortality and morbidity? Clin Obstet Gynecol. 1998 Mar;41(1):78-83. 
71. Larroque, B., Bréart, G., et al., Survival of very preterm infants: Epipage, a population based cohort study. Arch Dis Child Fetal Neonatal Ed. 2004 Mar; 89(2):F139-44.

72. Lacroze, V., Prématurité : définitions, épidémiologie, étiopathogénie, organisation des soins, in EMC (Elsevier Masson SAS, Paris). 2011. p. 4-002-N-10.

73. Ananth, C.V., Vintzileos, A.M., Medically incated preterm birth: recognizing the importance of the problem. Clin Perinatal 2008;35:53-67.

74. Fournié, A., et al., Hypotrophie, retard de croissance intra-utérin, souffrance foetale chronique in EMC (Elsevier Masson SAS, Paris). 2004. p. 5-076-E-10.

75. Malchau, S.S., et al., Perinatal outcomes in 6,338 singletons born after intrauterine insemination in Denmark, 2007 to 2012: the influence of ovarian stimulation. Fertility and Sterility, Vol. 102, No. 4, October 2014 0015-0282.

76. Helmerhorst, F.M., et al., Perinatal outcome of singletons and twins after assisted conception: a systematic review of controlled studies. Bmj, 2004. 328(7434): p. 261.

77. McDonald, S.D., et al., Preterm birth and low birth weight among in vitro fertilization singletons: a systematic review and meta-analyses. Eur J Obstet Gynecol Reprod Biol, 2009. 146(2): p. 138-48.

78. Henningsen, A.K., et al., Perinatal outcome of singleton siblings born after assisted reproductive technology and spontaneous conception: Danish national sibling-cohort study. Fertility and Sterility Vol. 95, No. 3, March 1, 2011, p959-963.

79. Pandey, S., et al., Obstetric and perinatal outcomes in singleton pregnancies resulting from IVF/ICSI: a systematic review and meta-analysis. Human Reproduction, Vol.18, No.5 pp. 485-503, 2012.

80. Larroque, B., et al., Neurodevelopmental disabilities and special care of 5-year-old children born before 33 weeks of gestation (the EPIPAGE study): a longitudinal cohort study. Lancet, 2008. 371(9615): p. 813-20.

81. Institute of Medicine Committee on Understanding Premature, B. and O.B. Assuring Healthy, R.E., Butler, A.S., editors, Preterm Birth: Causes, Consequences, and Prevention. 2007, National Academies Press (US), National Academy of Sciences: Washington (DC).

82. Amor, D.J., Halliday, J., A review of known imprinting syndromes and their association with assisted reproduction technologies. Hum Reprod, 2008. 23(12): p. 2826-34.

83. Shufaro, Y., Laufer, N., Epigenetic concerns in assisted reproduction: update and critical review of the current literature. Fertility and Sterility, Volume 99, Issue 3, 1 March 2013, Pages 605-606.

84. Fauser, B.C., et al., Health outcomes of children born after IVF/ICSI: a review of current expert opinion and literature. Reprod Biomed Online, 2014. 28(2): p. 162-82.

85. Hart, R., Norman, R.J., The longer-term health outcomes for children born as a result of IVF treatment: Part I--General health outcomes.Hum Reprod Update, 2013. 19(3): p. 232-43.

86. Thierry, X., et al., La cohorte Elfe : de quels enfants est-elle et sera-t-elle représentative?, in colloque pluridisciplinaire international d'Amiens. 2014: Université de Picardie, Amiens. 
87. Présentation générale de l'étude Elfe, Plateforme d'accès aux données RE-CO-NAI: 18/12/2012, https://pandora.vjf.inserm.fr/public/docs/presentation_generale_elfe.pdf, consulté le 17/07/2015.

88. Etude épidémiologique sur les petits âges gestationnels, http://epipage2.inserm.fr.

89. Protocole de l'enquête Elfe en maternité, Plateforme RE-CO-NAI 19/12/2012, https://pandora.vjf.inserm.fr/public/docs/Protocole_enquete_en_maternite.pdf, consulté le 17/07/2015.

90. Bilan synthétique du recrutement dans l'enquête Elfe et des différentes phases de collecte en maternité, Plateforme RE-CO-NAI 17/09/2014,

https://pandora.vjf.inserm.fr/public/docs/Bilan_enquete_Elfe_en_maternite.pdf, consulté le 17/07/2015.

91. Protocole et réalisation de l'enquête Elfe « deux mois », Plateforme RE-CO-NAI 27/01/2014, https://pandora.vjf.inserm.fr/public/docs/Protocole2Mois.pdf, consulté le $17 / 07 / 2015$.

92. Bilan synthétique de l'enquête 2 mois, Coordination Elfe 24 Janvier 2014, https://pandora.vjf.inserm.fr/public/docs/BS2Mois.pdf, consulté le 17/07/2015.

93. Juillard, H., Thierry, X., Pondération de l'enquête ELFE en maternité. Plateforme RE-CO-NAI janvier 2013 (actualisation : avril 2015), https://pandora.vjf.inserm.fr/public/ docs/Pondération_0.pdf, consulté le 17/07/2015.

94. Juillard, H., Thierry X., Pondérations de l'enquête ELFE au temps 1 (2 mois de l'enfant). Plateforme RE-CO-NAI mars 2014 (actualisation juillet 2015), https://pandora.vjf.inserm.fr/public/docs/ELFE_Note_Poids2M.pdf, consulté le $17 / 07 / 2015$.

95. InVS, Malformations congénitales et anomalies chromosomiques. Dossier thématique publié le 09/04/2014 - Dernière mise à jour le 02/03/2015.

http://www.invs.sante.fr/Dossiers-thematiques/Maladies-chroniques-et-traumatismes /Malformations-congenitales-et-anomalies-chromosomiques

96. Hammarberg, K., et al., Psychological and social aspects of pregnancy, childbirth and early parenting after assisted conception : a systematic review. Hum Reprod Update. 2008 Sep-Oct;14(5):395-414.

97. Monti, F., et al., Anxiety symptoms during late pregnancy and early parenthood following assisted reproductive technology. J Perinat Med. 2008;36(5):425-32.

98. Agostini, F., et al., Parental Mental Representations during late pregnancy and early parenthood following assisted reproductive technology. J Perinat Med. 2008;36(5):425-32.

99. Kowalcek, I., Experience of pregnancy and delivery after ART. Z Geburtshilfe Neonatol. 2011 Oct;215(5):183-6.

100. D'Angelo, D.V., et al., Birth outcomes of intended pregnancies among women who used assisted reproductive technology, ovulation stimulation, or no treatment. Fertility and Sterility, 2011, Vol. 96, No. 2, p. 314-320.

101. Hayashi, M., et al., Adverse obstetric and perinatal outcomes of singleton pregnancies may be related to maternal factors associated with infertility rather than the type of assisted reproductive technology procedure used. Fertility and Sterility, 2012, Vol. 98, No. 4, p. 922-928. 
102. Erikson, R., et al., On class differentials in educational attainment. Proc Natl Acad Sci U S A, 2005. 102(27): p. 9730-3.

103. Buis, M.L., Direct and indirect effects in a logit model. The Stata Journal, 2010. 10(Number 1): p. 11-29.

104. Pinborg, A., et al., Why do singletons conceived after assisted reproduction technology have adverse perinatal outcome? Systematic review and meta-analysis. Hum Reprod Update, 2013. 19(2): p. 87-104.

105. Morken, N.H., Preterm delivery in IVF versus ICSI singleton pregnancies: a national population-based cohort. Eur J Obstet Gynecol Reprod Biol, 2011. 154(1): p. 62-6

106. Ireton, H., Glascoe, F.P., Assessing children's development using parents' reports. The Child Development Inventory. Clin Pediatr, 1995. 34: pp. 248-255.

107. Duyme, M., Capron, C., L'Inventaire du Développement de l'Enfant (IDE). Normes et validation françaises du Child Development Inventory (CDI). Devenir, 2010/1 (Vol. 22), Pages 13 - 26.

108. Fenson, L., et al., MacArthur Communicative Development Inventories: User's guide and technical manual. 1993. San Diego, CA Singular Publishing Group.

109. Elliott, C.D., et al., British Ability Scales Second Edition (BAS II): technical manual. 1997. London: NFER-Nelson.

110. Hill, V., Through the past darkly: a review of the British Ability Scales Second Edition. Child Adolesc Ment Health. 2005;10:87-98. 



\section{ANNEXES}

Tableau A1 : Caractéristiques sociodémographiques parentales selon le contexte de la conception

Tableau A2 : Antécédents maternels et contexte de la grossesse selon le contexte de la conception

Tableau A3 : Pathologies et complications pendant la grossesse selon le contexte de la conception

Tableau A4 : Travail et accouchement, état de santé des mères et des enfants à la naissance selon le contexte de la conception

Tableau A5 : État de santé des enfants à deux mois selon le contexte de la conception

Tableau A6 : Caractéristiques sociodémographiques parentales selon le groupe de traitement de l'infertilité

Tableau A7 : Antécédents maternels et contexte de la grossesse selon le groupe de traitement de l'infertilité

Tableau A8 : Pathologies et complications de la grossesse selon le groupe de traitement de l'infertilité

Tableau A9 : Travail et accouchement, état de santé des mères et des enfants à la naissance selon le groupe de traitement de l'infertilité

Tableau A10 : État de santé des enfants à deux mois selon le groupe de traitement de l'infertilité

Tableau A11 : Analyse des facteurs associés à la prématurité et au petit poids de naissance selon le groupe de traitement de l'infertilité, modèles logistiques complets 

Tableau A1 : Caractéristiques sociodémographiques parentales selon le contexte de la conception

\begin{tabular}{|c|c|c|c|c|c|c|c|}
\hline & \multirow{2}{*}{$\begin{array}{c}\text { groupe traitement } \\
(\mathrm{N}=790) \\
\%\end{array}$} & \multirow{2}{*}{$\begin{array}{c}\text { groupe infertile } \\
(\mathrm{N}=1044) \\
\%\end{array}$} & \multirow{2}{*}{$\begin{array}{c}\text { groupe fertile } \\
(\mathrm{N}=7661) \\
\%\end{array}$} & \multicolumn{4}{|c|}{$p$} \\
\hline & & & & global & $\begin{array}{l}\text { traitement } \\
\text { vs infertile }\end{array}$ & $\begin{array}{l}\text { traitement } \\
\text { vs fertile }\end{array}$ & $\begin{array}{l}\text { infertile } \\
\text { vs fertile }\end{array}$ \\
\hline âge maternel à la naissance & & & & $<0,001$ & $<0,001$ & $<0,001$ & $<0,001$ \\
\hline$<25$ ans & 2,4 & 9,6 & 12,5 & & & & \\
\hline $25-35$ ans & 65,5 & 61,5 & 72,5 & & & & \\
\hline$\geq 35$ ans & 32,1 & 28,8 & 15,1 & & & & \\
\hline mère française de naissance & 89,9 & 81,7 & 91,0 & $<0,001$ & $<0,001$ & 0,485 & $<0,001$ \\
\hline diplôme le plus élevé obtenu par la mère & & & & $<0,001$ & $<0,001$ & 0,004 & $<0,001$ \\
\hline$\leq$ brevet des collèges & 4,2 & 9,9 & 7,1 & & & & \\
\hline$C A P / B E P$ & 11,2 & 17,8 & 13,7 & & & & \\
\hline baccalauréat & 13,8 & 18,6 & 17,6 & & & & \\
\hline$b a c+2$ & 27,3 & 22,1 & 23,1 & & & & \\
\hline$>b a c+2$ & 43,5 & 31,6 & 38,6 & & & & \\
\hline CSP maternelle élevée & 56,6 & 45,8 & 49,4 & $<0,001$ & $<0,001$ & 0,001 & 0,055 \\
\hline emploi lors de la grossesse & 88,9 & 74,4 & 80,0 & $<0,001$ & $<0,001$ & $<0,001$ & 0,003 \\
\hline couverture maladie maternelle & & & & $<0,001$ & $<0,001$ & 0,069 & $<0,001$ \\
\hline$C M U / A M E$ / aucun régime & 2,5 & 9,2 & 4,9 & & & & \\
\hline régime sans complémentaire & 4,8 & 6,6 & 5,5 & & & & \\
\hline régime et complémentaire & 92,7 & 84,2 & 89,6 & & & & \\
\hline ressources mensuelles du foyer & & & & $<0,001$ & $<0,001$ & $<0,001$ & $<0,001$ \\
\hline$<2500$ euros & 16,5 & 34,4 & 25,4 & & & & \\
\hline $2500-3999$ euros & 46,3 & 42,3 & 49,4 & & & & \\
\hline$\geq 4000$ euros & 37,2 & 23,3 & 25,2 & & & & \\
\hline état matrimonial : mère mariée ou pacsée & 80,5 & 63,6 & 62,4 & $<0,001$ & $<0,001$ & $<0,001$ & 0,561 \\
\hline vie en couple à la naissance & 99,9 & 98,4 & 98,9 & 0,022 & 0,003 & 0,016 & 0,198 \\
\hline
\end{tabular}


Tableau A2 : Antécédents maternels et contexte de la grossesse selon le contexte de la conception

\begin{tabular}{|c|c|c|c|c|c|c|c|}
\hline & \multirow{2}{*}{$\begin{array}{c}\text { groupe traitement } \\
(\mathrm{N}=790) \\
\% \\
\end{array}$} & \multirow{2}{*}{$\begin{array}{c}\text { groupe infertile } \\
(\mathrm{N}=1044) \\
\% \\
\end{array}$} & \multirow{2}{*}{$\begin{array}{c}\text { groupe fertile } \\
(\mathrm{N}=7661) \\
\% \\
\end{array}$} & \multicolumn{4}{|c|}{$\mathrm{p}$} \\
\hline & & & & global & $\begin{array}{l}\text { traitement } \\
\text { vs infertile }\end{array}$ & $\begin{array}{c}\text { traitement } \\
\text { vs fertile }\end{array}$ & $\begin{array}{l}\text { infertile } \\
\text { vs fertile }\end{array}$ \\
\hline grossesse antérieure & 60,6 & 67,0 & 64,5 & 0,045 & 0,012 & 0,050 & 0,170 \\
\hline fausse couche pendant la période à essayer d'être enceinte & 27,1 & 38,9 & 10,9 & & $<0,001$ & & \\
\hline IVG antérieure & 15,9 & 23,4 & 22,2 & 0,046 & 0,019 & 0,019 & 0,566 \\
\hline nombre d'enfants antérieurs & & & & $<0,001$ & 0,002 & $<0,001$ & $<0,001$ \\
\hline 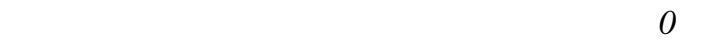 & 63,9 & 57,5 & 47,9 & & & & \\
\hline 1 & 28,8 & 28,8 & 36,7 & & & & \\
\hline$\geq 2$ & 7,3 & 13,7 & 15,4 & & & & \\
\hline HTA (chronique ou gestationnelle) & 1,3 & 2,3 & 2,2 & 0,398 & 0,178 & 0,144 & 0,814 \\
\hline diabète (chronique ou gestationnel) & 3,0 & 3,9 & 3,1 & 0,522 & 0,414 & 0,917 & 0,324 \\
\hline IMC maternel avant grossesse $\left(\mathrm{kg} / \mathrm{m}^{2}\right)$ & & & & 0,007 & 0,015 & 0,120 & 0,011 \\
\hline$<18,5$ & 8,6 & 9,6 & 7,1 & & & & \\
\hline $18,5-24,9$ & 69,2 & 61,7 & 66,4 & & & & \\
\hline $25-29,9$ & 15,1 & 17,0 & 17,4 & & & & \\
\hline$\geq 30$ & 7,1 & 11,7 & 9,0 & & & & \\
\hline antécédent de tabagisme avant la grossesse & 36,7 & 41,2 & 42,5 & 0,033 & 0,094 & 0,007 & 0,523 \\
\hline tabagisme pendant la grossesse & 11,2 & 18,6 & 18,2 & $<0,001$ & $<0,001$ & $<0,001$ & 0,789 \\
\hline tabagisme pendant le $3^{\text {ème }}$ trimestre & 8,8 & 15,0 & 14,2 & 0,003 & 0,002 & $<0,001$ & 0,570 \\
\hline consommation d'alcool lors de la grossesse & 12,6 & 14,9 & 18,8 & $<0,001$ & 0,204 & $<0,001$ & 0,007 \\
\hline acide folique avant/pendant la grossesse & 70,7 & 48,4 & 43,5 & $<0,001$ & $<0,001$ & $<0,001$ & 0,014 \\
\hline
\end{tabular}


Tableau A3 : Pathologies et complications pendant la grossesse selon le contexte de la conception

\begin{tabular}{|c|c|c|c|c|c|c|c|}
\hline & \multirow{2}{*}{$\begin{array}{c}\text { groupe traitement } \\
(\mathrm{N}=790) \\
\%\end{array}$} & \multirow{2}{*}{$\begin{array}{c}\text { groupe infertile } \\
(\mathrm{N}=1044) \\
\%\end{array}$} & \multirow{2}{*}{$\begin{array}{c}\text { groupe fertile } \\
\text { (N=7 661) } \\
\%\end{array}$} & \multicolumn{4}{|c|}{$\mathrm{p}$} \\
\hline & & & & global & $\begin{array}{l}\text { traitement } \\
\text { vs infertile }\end{array}$ & $\begin{array}{c}\text { traitement } \\
\text { vs fertile }\end{array}$ & $\begin{array}{l}\text { infertile } \\
\text { vs fertile }\end{array}$ \\
\hline mère heureuse d'être enceinte & 99,2 & 96,0 & 94,8 & $<0,001$ & $<0,001$ & $<0,001$ & 0,162 \\
\hline difficultés psychologiques & 12,2 & 14,0 & 9,9 & $<0,001$ & 0,332 & 0,063 & $<0,001$ \\
\hline hospitalisation pendant la grossesse & 24,2 & 20,9 & 14,7 & $<0,001$ & 0,176 & $<0,001$ & $<0,001$ \\
\hline pathologie de la grossesse & 32,0 & 27,4 & 23,9 & $<0,001$ & 0,087 & $<0,001$ & 0,054 \\
\hline diabète gestationnel & 9,3 & 8,1 & 6,3 & 0,010 & 0,480 & 0,011 & 0,051 \\
\hline placenta previa hémorragique & 1,3 & 0,5 & 0,3 & $<0,001$ & 0,047 & $<0,001$ & 0,343 \\
\hline $\begin{array}{l}\text { menace d'accouchement prématuré } \\
\text { corticothérapie anténatale }\end{array}$ & $\begin{array}{c}11,1 \\
8,9\end{array}$ & $\begin{array}{l}9,7 \\
6,0\end{array}$ & $\begin{array}{l}7,3 \\
3,6\end{array}$ & $\begin{array}{c}0,002 \\
<0,001\end{array}$ & $\begin{array}{l}0,443 \\
0,056\end{array}$ & $\begin{array}{c}0,002 \\
<0,001\end{array}$ & $\begin{array}{l}0,030 \\
0,002\end{array}$ \\
\hline pathologie foetale & 4,2 & 3,1 & 3,2 & 0,448 & 0,285 & 0,204 & 0,867 \\
\hline
\end{tabular}


Tableau A4 : Travail et accouchement, état de santé des mères et des enfants à la naissance selon le contexte de la conception

\begin{tabular}{|c|c|c|c|c|c|c|c|}
\hline & \multirow{2}{*}{$\begin{array}{c}\text { groupe traitement } \\
(\mathrm{N}=790) \\
\%\end{array}$} & \multirow{2}{*}{$\begin{array}{c}\text { groupe infertile } \\
(\mathrm{N}=1044) \\
\%\end{array}$} & \multirow{2}{*}{$\begin{array}{c}\text { groupe fertile } \\
(\mathrm{N}=7661) \\
\%\end{array}$} & \multicolumn{4}{|c|}{$\mathrm{p}$} \\
\hline & & & & global & $\begin{array}{l}\text { traitement } \\
\text { vs infertile }\end{array}$ & $\begin{array}{l}\text { traitement } \\
\text { vs fertile }\end{array}$ & $\begin{array}{l}\text { infertile } \\
\text { vs fertile }\end{array}$ \\
\hline gémellité & 12,3 & 1,0 & 1,2 & $<0,001$ & $<0,001$ & $<0,001$ & 0,528 \\
\hline travail spontané & 64,4 & 69,8 & 73,4 & $<0,001$ & 0,035 & $<0,001$ & 0,046 \\
\hline type d'accouchement & & & & $<0,001$ & 0,204 & $<0,001$ & $<0,001$ \\
\hline voie basse spontanée & 58,1 & 59,5 & 70,3 & & & & \\
\hline voie basse instrumentale & 14,0 & 16,4 & 13,2 & & & & \\
\hline césarienne & 27,9 & 24,1 & 16,5 & & & & \\
\hline prématurité & 10,4 & 6,4 & 4,1 & $<0,001$ & 0,010 & $<0,001$ & 0,007 \\
\hline petit poids de naissance & 9,2 & 5,2 & 4,0 & $<0,001$ & 0,010 & $<0,001$ & 0,137 \\
\hline sexe masculin & 52,4 & 51,4 & 50,7 & 0,723 & 0,722 & 0,439 & 0,719 \\
\hline anomalie congénitale & 2,4 & 2,1 & 2,6 & 0,711 & 0,703 & 0,862 & 0,348 \\
\hline réanimation en salle de naissance & 4,6 & 3,9 & 2,9 & 0,064 & 0,526 & 0,013 & 0,211 \\
\hline score d'Apgar à 5 minutes $\leq 7$ & 1,5 & 3,0 & 1,2 & $<0,001$ & 0,082 & 0,462 & 0,001 \\
\hline hospitalisation / transfert de la mère & 1,3 & 1,8 & 1,0 & 0,151 & 0,470 & 0,416 & 0,101 \\
\hline transfert de l'enfant & 8,0 & 7,4 & 4,9 & $<0,001$ & 0,711 & 0,002 & 0,005 \\
\hline traitement particulier en suites de couches & 8,4 & 9,0 & 6,7 & 0,027 & 0,721 & 0,123 & 0,018 \\
\hline antibiothérapie intraveineuse & 1,6 & 3,7 & 1,7 & $<0,001$ & 0,010 & 0,853 & $<0,001$ \\
\hline gavage & 3,2 & 2,1 & 1,4 & 0,020 & 0,341 & 0,008 & 0,166 \\
\hline allaitement maternel (complet ou mixte) & 71,4 & 70,2 & 67,7 & 0,104 & 0,633 & 0,068 & 0,181 \\
\hline
\end{tabular}


Tableau A5 : État de santé des enfants à deux mois selon le contexte de la conception

\begin{tabular}{|c|c|c|c|c|c|c|c|}
\hline & \multirow{3}{*}{$\begin{array}{c}\text { groupe traitement } \\
(\mathrm{N}=790) \\
\% \\
\end{array}$} & \multirow{3}{*}{$\begin{array}{c}\text { groupe infertile } \\
(\mathrm{N}=1044) \\
\% \\
\end{array}$} & \multirow{3}{*}{$\begin{array}{c}\text { groupe fertile } \\
(\mathrm{N}=7661) \\
\% \\
\end{array}$} & \multirow{2}{*}{\multicolumn{4}{|c|}{$p$}} \\
\hline & & & & & & & \\
\hline & & & & global & $\begin{array}{l}\text { traitement } \\
\text { vs infertile }\end{array}$ & $\begin{array}{c}\text { traitement } \\
\text { vs fertile }\end{array}$ & $\begin{array}{l}\text { infertile } \\
\text { vs fertile }\end{array}$ \\
\hline enfant en bonne santé à deux mois & 99,3 & 98,4 & 99,2 & 0,125 & 0,174 & 0,806 & 0,070 \\
\hline problème de santé dans les deux premiers mois & 74,5 & 75,4 & 71,1 & 0,018 & 0,702 & 0,089 & 0,017 \\
\hline prise de poids dans les deux premiers mois $(\mathrm{g} / \mathrm{j})$ & & & & 0,123 & 0,113 & 0,214 & 0,145 \\
\hline$<20 \mathrm{~g} / \mathrm{j}$ & 20,6 & 23,4 & 24,6 & & & & \\
\hline $20-40 \mathrm{~g} / \mathrm{j}$ & 72,3 & 65,7 & 67,5 & & & & \\
\hline$\geq 40 \mathrm{~g} / \mathrm{j}$ & 7,1 & 10,9 & 7,9 & & & & \\
\hline recours hospitalier dans les deux premiers mois & 16,1 & 17,3 & 14,9 & 0,185 & 0,557 & 0,418 & 0,102 \\
\hline consultation aux urgences & 12,0 & 15,0 & 11,8 & 0,036 & 0,111 & 0,827 & 0,018 \\
\hline hospitalisation & 7,2 & 4,6 & 5,8 & 0,084 & 0,024 & 0,164 & 0,110 \\
\hline bonne relation mère-enfant à deux mois & 76,0 & 74,4 & 77,7 & 0,102 & 0,510 & 0,354 & 0,050 \\
\hline la mère chante à l'enfant & 93,2 & 94,4 & 92,8 & 0,190 & 0,323 & 0,666 & 0,089 \\
\hline la mère parle à l'enfant & 99,4 & 98,4 & 99,2 & 0,106 & 0,066 & 0,461 & 0,098 \\
\hline l'enfant cherche le regard de sa mère & 96,1 & 95,0 & 95,3 & 0,645 & 0,355 & 0,353 & 0,780 \\
\hline l'enfant sourit à sa mère & 94,4 & 94,2 & 95,8 & 0,050 & 0,867 & 0,079 & 0,056 \\
\hline la mère connaît la cause des pleurs & 87,3 & 86,0 & 90,2 & $<0,001$ & 0,519 & 0,039 & 0,001 \\
\hline allaitement maternel (complet ou mixte) & 46,4 & 47,3 & 45,2 & 0,492 & 0,745 & 0,576 & 0,281 \\
\hline
\end{tabular}


Tableau A6 : Caractéristiques sociodémographiques parentales selon le groupe de traitement de l'infertilité

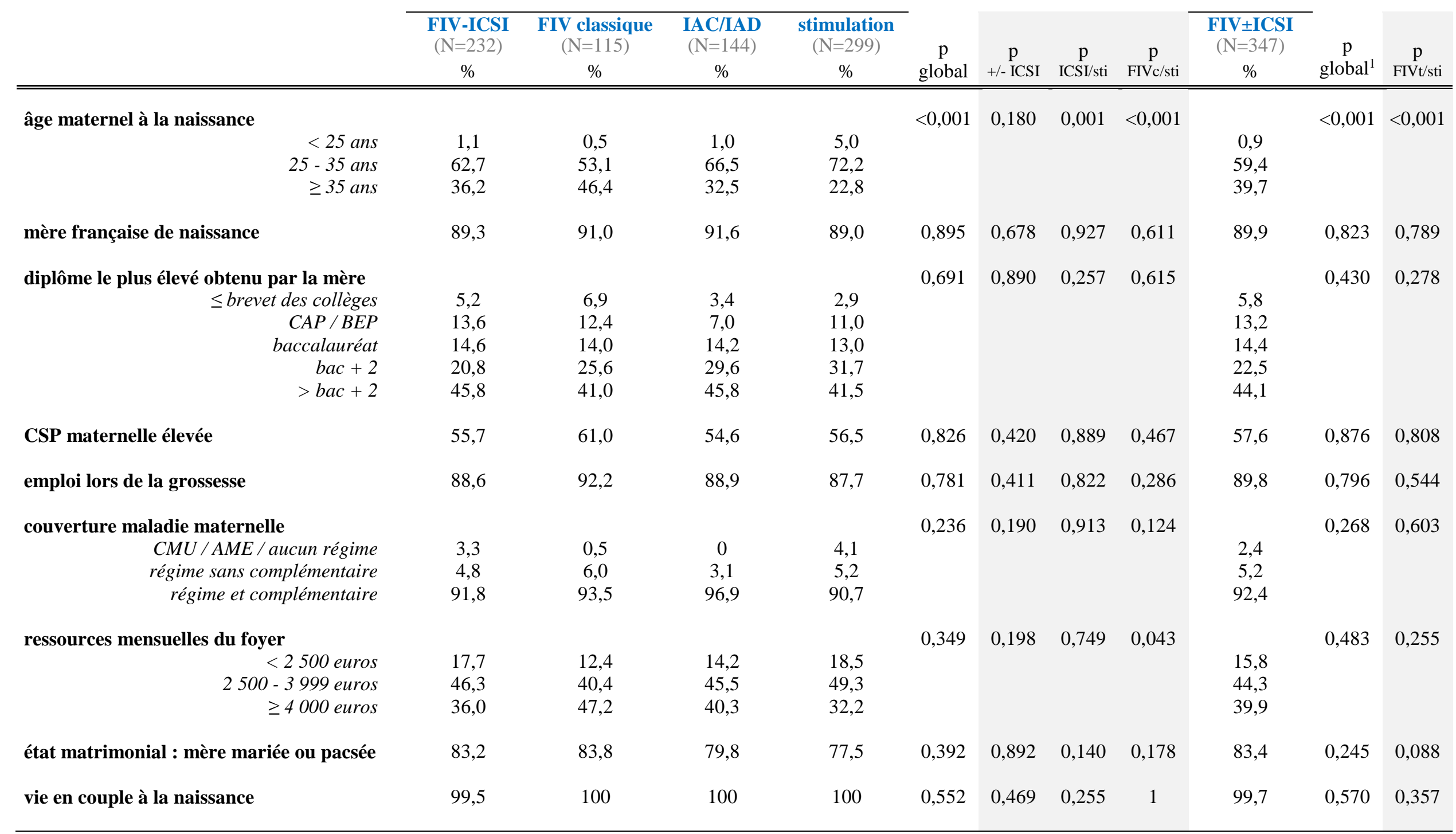

${ }^{1}$ comparaison FIV (avec ou sans ICSI) / insémination / stimulation. 
Tableau A7 : Antécédents maternels et contexte de la grossesse selon le groupe de traitement de l'infertilité

\begin{tabular}{|c|c|c|c|c|c|c|c|c|c|c|c|}
\hline & & & & & \multirow[b]{2}{*}{$\begin{array}{c}\mathrm{p} \\
\text { global } \\
\end{array}$} & \multirow[b]{2}{*}{$\begin{array}{c}\mathrm{p} \\
+/- \text { ICSI } \\
\end{array}$} & \multirow[b]{2}{*}{$\begin{array}{c}\mathrm{p} \\
\mathrm{ICSI} / \mathrm{sti} \\
\end{array}$} & \multirow[b]{2}{*}{$\begin{array}{c}\mathrm{p} \\
\text { FIVc/sti } \\
\end{array}$} & \multirow[b]{2}{*}{$\begin{array}{c}\mathbf{F I V} \pm \mathbf{I C S I} \\
(\mathrm{N}=347) \\
\% \\
\end{array}$} & \multirow[b]{2}{*}{$\begin{array}{c}\mathrm{p} \\
\text { global }\end{array}$} & \multirow[b]{2}{*}{$\underset{\text { FIVt/sti }}{\mathrm{p}}$} \\
\hline & $\begin{array}{c}\text { FIV-ICSI } \\
(\mathrm{N}=232) \\
\% \\
\end{array}$ & $\begin{array}{c}\text { FIV classique } \\
(\mathrm{N}=115) \\
\% \\
\end{array}$ & $\begin{array}{c}\text { IAC/IAD } \\
(\mathrm{N}=144) \\
\% \\
\end{array}$ & $\begin{array}{c}\text { stimulation } \\
(\mathrm{N}=299) \\
\% \\
\end{array}$ & & & & & & & \\
\hline grossesse antérieure & 60,0 & 56,9 & 59,4 & 63,2 & 0,740 & 0,625 & 0,495 & 0,304 & 58,9 & 0,603 & 0,314 \\
\hline nombre d'enfants antérieurs & & & & & 0,111 & 0,533 & 0,007 & 0,680 & & 0,035 & 0,061 \\
\hline 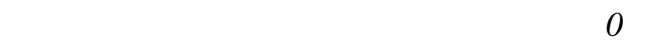 & 69,3 & 62,5 & 70,4 & 56,8 & & & & & 66,8 & & \\
\hline 1 & 26,4 & 29,9 & 25,8 & 31,8 & & & & & 27,7 & & \\
\hline$\geq 2$ & 4,3 & 7,6 & 3,8 & 11,3 & & & & & 5,5 & & \\
\hline HTA (chronique ou gestationnelle) & 0,8 & 3,7 & 1,0 & 0,9 & 0,152 & 0,058 & 0,909 & 0,056 & 1,8 & 0,609 & 0,327 \\
\hline diabète (chronique ou gestationnel) & 2,4 & 2,0 & 1,1 & 5,0 & 0,097 & 0,793 & 0,182 & 0,144 & 2,3 & 0,044 & 0,083 \\
\hline IMC maternel avant grossesse $\left(\mathrm{kg} / \mathrm{m}^{2}\right)$ & & & & & 0,080 & 0,075 & 0,511 & 0,065 & & 0,210 & 0,344 \\
\hline$<18,5$ & 10,2 & 5,4 & 3,8 & 11,3 & & & & & 8,5 & & \\
\hline $18,5-24,9$ & 66,2 & 80,5 & 73,3 & 64,7 & & & & & 71,1 & & \\
\hline $25-29,9$ & 18,2 & 8,0 & 16,0 & 15,0 & & & & & 14,7 & & \\
\hline$\geq 30$ & 5,5 & 6,1 & 6,9 & 9,0 & & & & & 5,7 & & \\
\hline antécédent de tabagisme avant la grossesse & 32,5 & 44,9 & 44,9 & 31,9 & 0,030 & 0,054 & 0,904 & 0,037 & 36,8 & 0,073 & 0,255 \\
\hline tabagisme pendant la grossesse & 8,1 & 15,9 & 15,8 & 9,2 & 0,116 & 0,107 & 0,677 & 0,164 & 10,8 & 0,192 & 0,560 \\
\hline tabagisme pendant le $3^{\text {ème }}$ trimestre & 7,1 & 15,9 & 11,1 & 5,9 & 0,047 & 0,059 & 0,599 & 0,017 & 10,2 & 0,164 & 0,094 \\
\hline consommation d'alcool lors de la grossesse & 9,9 & 15,8 & 15,5 & 11,7 & 0,394 & 0,132 & 0,526 & 0,292 & 12,0 & 0,577 & 0,913 \\
\hline acide folique avant/pendant la grossesse & 78,1 & 73,2 & 76,5 & 60,5 & $<0,001$ & 0,380 & $<0,001$ & 0,036 & 76,5 & $<0,001$ & $<0,001$ \\
\hline
\end{tabular}


Tableau A8 : Pathologies et complications de la grossesse selon le groupe de traitement de l'infertilité

\begin{tabular}{|c|c|c|c|c|c|c|c|c|c|c|c|}
\hline & $\begin{array}{c}\text { FIV-ICSI } \\
(\mathrm{N}=232) \\
\%\end{array}$ & $\begin{array}{c}\text { FIV classique } \\
\begin{array}{c}(\mathrm{N}=115) \\
\%\end{array}\end{array}$ & $\begin{array}{c}\text { IAC/IAD } \\
(\mathrm{N}=144) \\
\%\end{array}$ & $\begin{array}{c}\text { stimulation } \\
(\mathrm{N}=299) \\
\%\end{array}$ & $\begin{array}{c}\mathrm{p} \\
\text { global }\end{array}$ & $\underset{\text { +/- ICSI }}{p}$ & $\underset{\text { ICSI/sti }}{p}$ & $p_{\mathrm{FIVc} / \mathrm{sti}}^{\mathrm{p}}$ & $\begin{array}{c}\mathbf{F I V} \pm \mathbf{I C S I} \\
(\mathrm{N}=347) \\
\%\end{array}$ & $\begin{array}{c}\mathrm{p} \\
\text { global }\end{array}$ & $\underset{\text { FIVt/sti }}{p}$ \\
\hline mère heureuse d'être enceinte & 100 & 99,5 & 100 & 97,9 & 0,049 & 0,170 & 0,041 & 0,149 & 99,8 & 0,009 & 0,003 \\
\hline difficultés psychologiques & 14,7 & 10,1 & 9,0 & 13,0 & 0,441 & 0,340 & 0,612 & 0,516 & 13,1 & 0,430 & 0,981 \\
\hline hospitalisation pendant la grossesse & 28,8 & 22,4 & 30,2 & 18,1 & 0,042 & 0,252 & 0,011 & 0,355 & 26,6 & 0,038 & 0,022 \\
\hline pathologie de la grossesse & 32,4 & 37,6 & 37,6 & 26,3 & 0,171 & 0,461 & 0,176 & 0,087 & 34,2 & 0,102 & 0,067 \\
\hline HTA apparue pendant la grossesse & 4,5 & 5,6 & 4,1 & 3,4 & 0,807 & 0,668 & 0,499 & 0,316 & 4,9 & 0,689 & 0,335 \\
\hline diabète gestationnel & 10,4 & 8,7 & 7,1 & 9,8 & 0,829 & 0,763 & 0,842 & 0,834 & 9,8 & 0,686 & 0,992 \\
\hline placenta pravia hémorragique & 2,3 & 3,5 & 0 & 0,3 & 0,010 & 0,456 & 0,040 & 0,007 & 2,7 & 0,007 & 0,016 \\
\hline $\begin{array}{l}\text { menace d'accouchement prématuré } \\
\text { corticothérapie anténatale }\end{array}$ & $\begin{array}{l}8,0 \\
7,2\end{array}$ & $\begin{array}{l}14,7 \\
11,8\end{array}$ & $\begin{array}{l}18,9 \\
14,9\end{array}$ & $\begin{array}{l}7,7 \\
5,5\end{array}$ & $\begin{array}{l}0,008 \\
0,035\end{array}$ & $\begin{array}{l}0,064 \\
0,173\end{array}$ & $\begin{array}{l}0,912 \\
0,446\end{array}$ & $\begin{array}{l}0,042 \\
0,039\end{array}$ & $\begin{array}{c}10,3 \\
8,8\end{array}$ & $\begin{array}{l}0,015 \\
0,039\end{array}$ & $\begin{array}{l}0,267 \\
0,135\end{array}$ \\
\hline pathologie fœetale & 1,5 & 4,3 & 10,4 & 2,9 & 0,002 & 0,100 & 0,365 & 0,530 & 2,5 & 0,003 & 0,827 \\
\hline suspicion de RCIU & 1,4 & 4,2 & 8,8 & 2,6 & 0,011 & 0,093 & 0,431 & 0,468 & 2,4 & 0,013 & 0,923 \\
\hline
\end{tabular}


Tableau A9 : Travail et accouchement, état de santé des mères et des enfants à la naissance selon le groupe de traitement de l'infertilité

\begin{tabular}{|c|c|c|c|c|c|c|c|c|c|c|c|}
\hline & $\begin{array}{c}\text { FIV-ICSI } \\
(\mathrm{N}=232) \\
\% \\
\end{array}$ & $\begin{array}{c}\text { FIV classique } \\
(\mathrm{N}=115) \\
\%\end{array}$ & $\begin{array}{c}\text { IAC/IAD } \\
(\mathrm{N}=144) \\
\% \\
\end{array}$ & $\begin{array}{c}\text { stimulation } \\
(\mathrm{N}=299) \\
\% \\
\end{array}$ & $\begin{array}{c}\mathrm{p} \\
\text { global }\end{array}$ & $\begin{array}{c}\mathrm{p} \\
+/ \text { ICSI } \\
\end{array}$ & $\begin{array}{c}\mathrm{p} \\
\text { ICSI/sti }\end{array}$ & $\begin{array}{c}\mathrm{p} \\
\text { FIV } \mathrm{c} / \mathrm{sti} \\
\end{array}$ & $\begin{array}{c}\text { FIV } \pm \text { ICSI } \\
(\mathrm{N}=347) \\
\% \\
\end{array}$ & $\begin{array}{c}\mathrm{p} \\
\text { global }\end{array}$ & $\mathrm{p}_{\text {FIVt } / \mathrm{sti}}$ \\
\hline gémellité & 14,3 & 19,3 & 17,6 & 4,9 & 0,001 & 0,352 & $<0,001$ & $<0,001$ & 16,1 & $<0,001$ & $<0,001$ \\
\hline travail spontané & 54,7 & 57,8 & 72,9 & 70,0 & 0,002 & 0,645 & 0,002 & 0,047 & 55,8 & $<0,001$ & 0,001 \\
\hline type d'accouchement & & & & & 0,003 & 0,990 & $<0,001$ & 0,017 & & $<0,001$ & $<0,001$ \\
\hline voie basse spontanée & 49,1 & 50,0 & 61,8 & 66,6 & & & & & 49,4 & & \\
\hline voie basse instrumentale & 14,2 & 13,9 & 17,0 & 12,2 & & & & & 14,1 & & \\
\hline césarienne & 36,7 & 36,1 & 21,2 & 21,2 & & & & & 36,5 & & \\
\hline prématurité & 12,3 & 16,0 & 10,7 & 6,4 & 0,115 & 0,479 & 0,022 & 0,019 & 13,6 & 0,060 & 0,006 \\
\hline petit poids de naissance & 6,8 & 17,1 & 13,7 & 5,2 & 0,009 & 0,019 & 0,506 & 0,005 & 10,4 & 0,057 & 0,059 \\
\hline sexe masculin & 51,8 & 59,1 & 45,3 & 54,0 & 0,278 & 0,296 & 0,662 & 0,448 & 54,4 & 0,249 & 0,930 \\
\hline anomalie congénitale & 1,0 & 2,0 & 6,6 & 1,4 & 0,010 & 0,426 & 0,682 & 0,616 & 1,4 & 0,007 & 0,967 \\
\hline réanimation en salle de naissance & 7,2 & 6,3 & 2,9 & 2,8 & 0,081 & 0,781 & 0,024 & 0,103 & 6,9 & 0,030 & 0,021 \\
\hline score d'Apgar à 5 minutes $\leq 7$ & 0,5 & 3,1 & 0 & 2,3 & 0,093 & 0,076 & 0,121 & 0,674 & 1,4 & 0,189 & 0,435 \\
\hline hospitalisation / transfert de la mère & 0,9 & 5,3 & 0,5 & 0,3 & $<0,001$ & 0,012 & 0,287 & $<0,001$ & 2,4 & 0,015 & 0,009 \\
\hline transfert de l'enfant & 6,5 & 15,0 & 7,6 & 6,3 & 0,083 & 0,049 & 0,931 & 0,035 & 9,5 & 0,438 & 0,209 \\
\hline traitement particulier en suites de couches & 7,3 & 16,0 & 6,2 & 7,3 & 0,057 & 0,052 & 0,991 & 0,043 & 10,4 & 0,292 & 0,253 \\
\hline photothérapie & 3,8 & 12,1 & 3,7 & 5,8 & 0,052 & 0,028 & 0,355 & 0,122 & 6,8 & 0,486 & 0,687 \\
\hline antibiothérapie intraveineuse & 1,9 & 1,5 & 1,9 & 1,1 & 0,880 & 0,815 & 0,429 & 0,705 & 1,8 & 0,732 & 0,448 \\
\hline gavage & 2,6 & 7,7 & 3,9 & 1,1 & 0,063 & 0,133 & 0,162 & 0,005 & 4,4 & 0,118 & 0,018 \\
\hline allaitement maternel (complet ou mixte) & 69,4 & 79,1 & 65,9 & 72,9 & 0,191 & 0,097 & 0,449 & 0,247 & 72,7 & 0,349 & 0,967 \\
\hline
\end{tabular}


Tableau A10 : État de santé des enfants à deux mois selon le groupe de traitement de l'infertilité

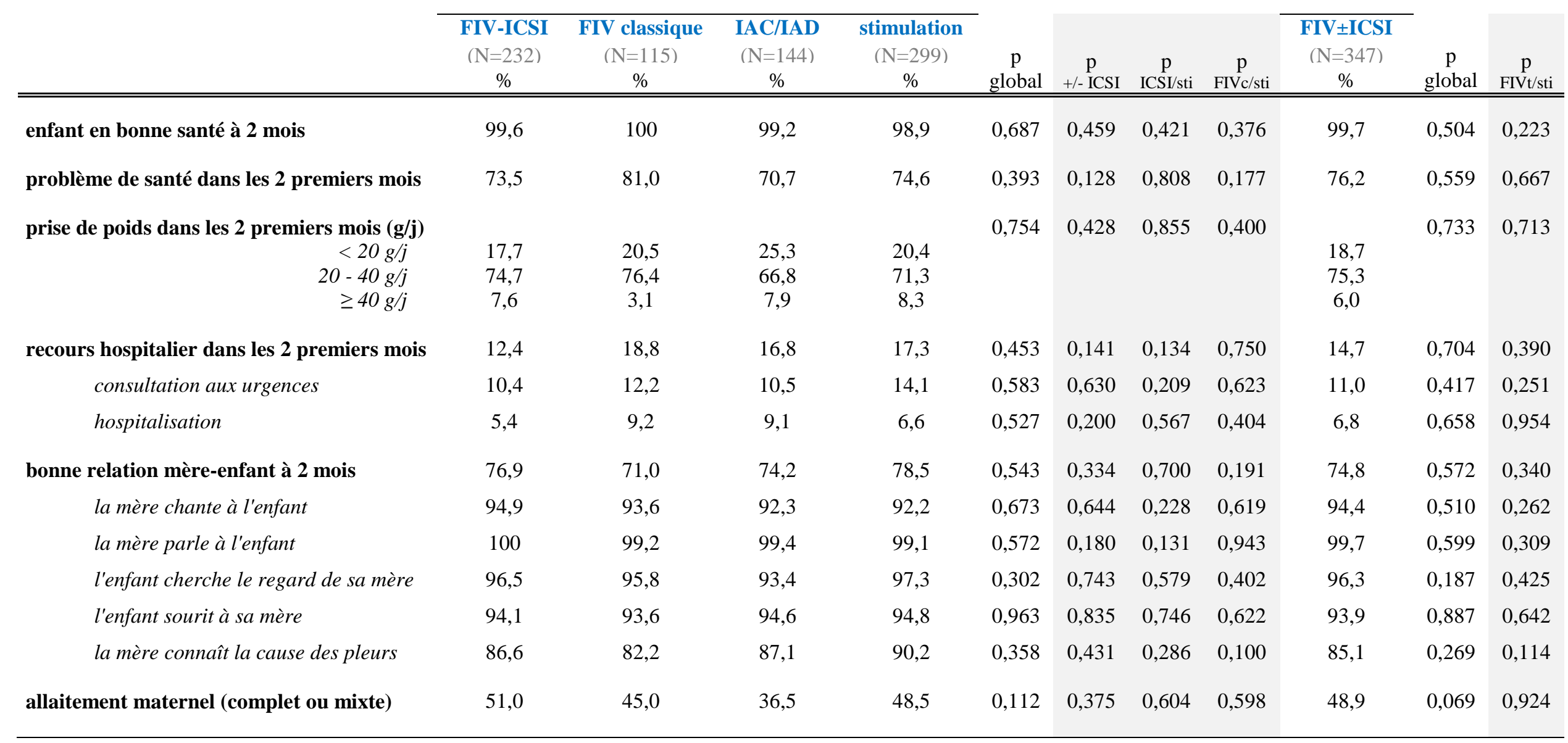


Tableau A11 : Analyse des facteurs associés à la prématurité / au petit poids de naissance selon le groupe de traitement de l'infertilité, modèles complets

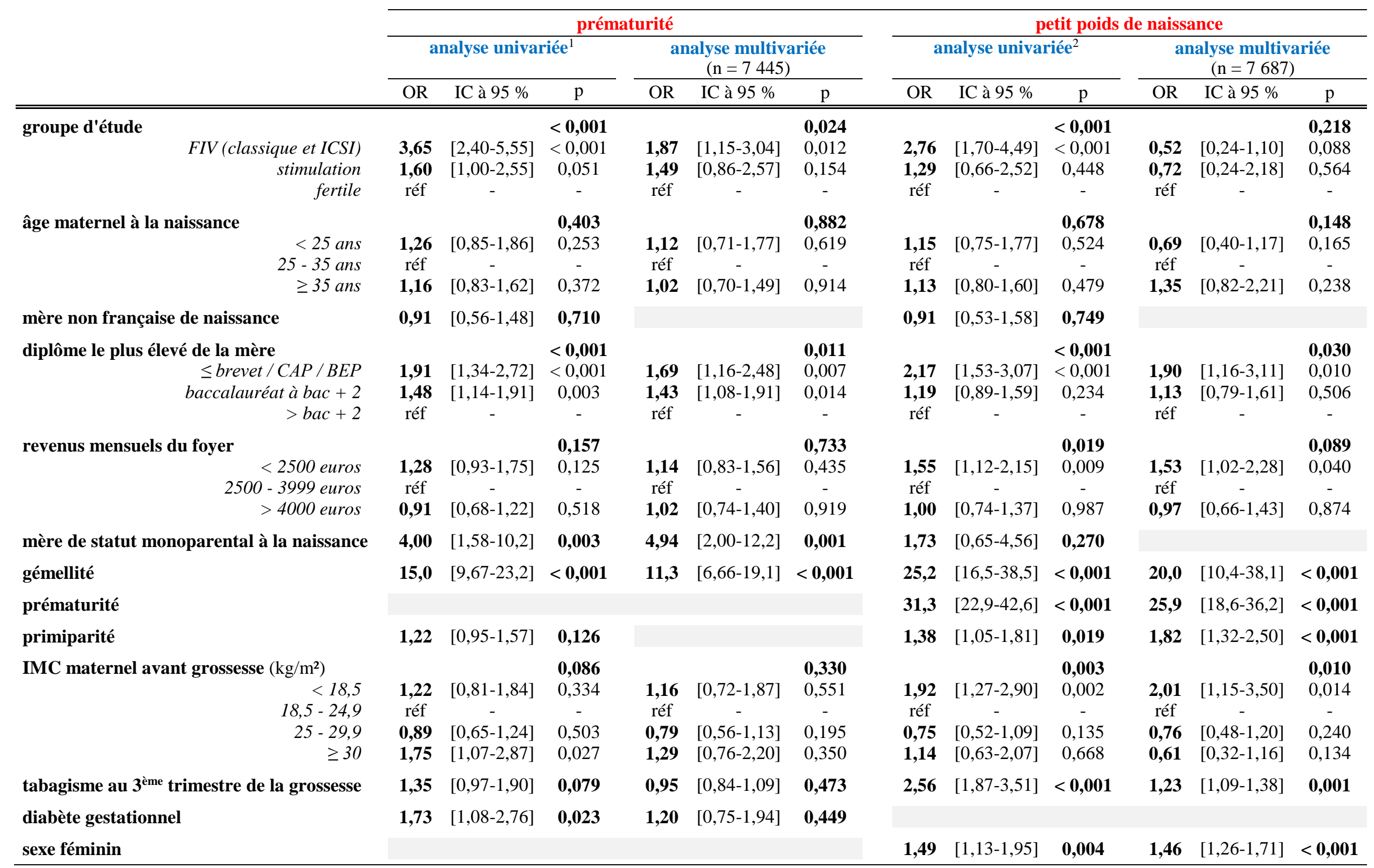

${ }^{1}$ n allant de 7819 à 8160 selon les variables. ${ }^{2} \mathrm{n}$ allant de 7856 à 9266 selon les variables. 




\title{
ENFANTS CONÇUS AVEC UNE AIDE MÉDICALE DANS LA COHORTE ELFE
}

Objectif : étudier les conditions entourant la naissance des enfants conçus avec une aide médicale, et explorer le rôle des facteurs sociaux et médicaux dans la prématurité et l'hypotrophie.

Méthodes : l'analyse porte sur 9495 enfants de la cohorte Elfe, de terme supérieur à 33 semaines d'aménorrhée, singletons ou jumeaux, répartis en trois groupes selon leur contexte de conception, "traitement", "infertiles non traités" et "fertiles" (790, 1044 et 7661 enfants). Cinq champs ont été décrits (caractéristiques sociales, antécédents maternels, grossesse, naissance, santé à deux mois). Une analyse univariée et multivariée des facteurs susceptibles d'être associés à la prématurité ou à l'hypotrophie a été réalisée. Les sous-groupes "fécondation in vitro" et "stimulation ovarienne" ont été comparés.

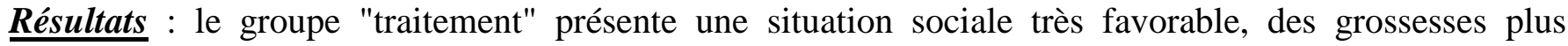
compliquées et une gémellité, une prématurité et une hypotrophie significativement plus fréquentes. La gémellité et la prématurité sont significativement plus fréquentes en FIV qu'en stimulation. Après ajustement, il existe une association significative entre contexte de conception et prématurité, sans différence entre groupes traitement et infertile ni entre groupes FIV et stimulation. Aucune association n'est retrouvée avec l'hypotrophie.

$\underline{\text { Conclusion }}$ : ces résultats suggèrent un sur-risque de complications néonatales chez les enfants de parents infertiles, traités ou non. Des particularités du groupe "infertile" empêchent cependant d'exclure un effet des techniques médicales. Le projet se poursuivra avec l'analyse du développement psychomoteur et pondéral entre la naissance et 3 ans.

MOTS-CLÉS : infertilité, assistance médicale à la procréation, stimulation ovarienne, prématurité, hypotrophie, petit poids de naissance, gémellité, contexte sociodémographique.

LABORATOIRE : INSERM CESP 1018, équipe 2 "Épidémiologie de la reproduction et du développement de l'enfant" (Jean Bouyer), Hôpital Kremlin Bicêtre, Secteur bleu - Pierre Lajaunias Porte 89, 82 rue du Général Leclerc, 94276 Le Kremlin-Bicêtre cedex, France.

\author{
Université Paris Descartes \\ Faculté de Médecine Paris Descartes \\ 15, rue de l'École de Médecine \\ 75270 Paris Cedex 06
}

